\title{
Chiral Metallacycles as Catalysts for Asymmetric Conjugate Addition of Styrylboronic Acids to $\alpha, \beta$-Enones
}

\author{
Tao Hong, ${ }^{\dagger}$ Zibin Zhang, ${ }^{\dagger}$ Yan Sun, ${ }^{\ddagger}$ Jia-Ju Tao, ${ }^{\dagger}$ Jia-Dong Tang, ${ }^{\dagger}$ Chunsong \\ Xie ${ }^{\dagger}$ Min Wang, ${ }^{\dagger}$ Fang Chen, ${ }^{\dagger}$ Shang-Shu Xie, ${ }^{\dagger}$ Shijun Li,,${ }^{*}$ and Peter J. Stang ${ }^{*}$, \\ ${ }^{\dagger}$ College of Material, Chemistry and Chemical Engineering, Hangzhou Normal \\ University, Hangzhou 311121, P. R. China \\ ${ }^{t}$ Department of Chemistry, University of Utah, 315 South 1400 East, Room 2020, Salt \\ Lake City, Utah 84112, United States
}

Email address: 1_shijun@hznu.edu.cn; stang@chem.utah.edu

1. Materials and methods $\quad S 2$

2. Synthesis of compound $(S)-2 \quad S 3$

3. Synthesis of compound (S)-3 $\quad S 4$

4. Synthesis of compound $(S)-4 \quad S 5$

5. Synthesis of compound (S)-5 S7

6. Self-assembly of chiral metalla-triangle (S)-7a S12

7. Self-assembly of chiral metalla-triangle $(S)-7 b \quad S 16$

8. General procedure for catalytic conjugate addition of $\alpha, \beta$-enones $\quad S 20$

9. ${ }^{1} H$ and ${ }^{13} C$ NMR spectra and chiral HPLC spectra of the addition products $\quad S 32$

10. References S68 


\section{Materials and methods}

Unless otherwise stated, chemicals and solvents were used as purchased from commercial sources and without further purification. THF was dried over sodium/benzophenone and freshly distilled under nitrogen atmosphere prior to use. Deuterated solvents were purchased from Cambridge Isotope Laboratory (Andover, MA). (S)-3,3'-Dibromo-1,1'-bi-2,2'-naphthol ((S)-11) was bought from $\mathrm{J} \& \mathrm{~K}$ Scientific Ltd. and used as received. The $180^{\circ}$ precursors $\mathbf{6 a}^{\mathrm{S} 1}$ and $\mathbf{6} \mathbf{b}^{\mathrm{S} 2}$ were prepared according to the literature procedures. NMR spectra were recorded on a Bruker AVANCE III $500 \mathrm{MHz}$ spectrometer at room temperature. ${ }^{1} \mathrm{H}$ NMR and ${ }^{13} \mathrm{C}$ NMR chemical shifts $(\delta)$ are given in ppm and are reported relative to tetramethylsilane (TMS) at $0.00 \mathrm{ppm}$ or the residual solvent signals. ${ }^{31} \mathrm{P}\left\{{ }^{1} \mathrm{H}\right\}$ NMR chemical shifts are referenced to an external unlocked sample of $85 \% \mathrm{H}_{3} \mathrm{PO}_{4}(\delta$ 0.0). Circular dichroism (CD) spectra were recorded on a JASCO J-815 circular dichroism chiroptical spectrometer in $\mathrm{CH}_{2} \mathrm{Cl}_{2}$ at room temperature. Mass spectra were recorded on an Agilent 1290-6530 UPLC-Q-TOF spectrometer using electrospray ionization or on a Waters GCT Premier GC-TOF spectrometer using electron impact ionization. Optical rotation analyses were performed on an MCP 500 optical instrument. HPLC analysis was carried out on an Agilent 1200 or an Agilent 1260 liquid chromatography equipped with a UV detector. 
2. Synthesis of compound (S)-2 ${ }^{\mathrm{S} 3}$<smiles>Oc1ccc2ccccc2c1-c1c(O)ccc2ccccc12</smiles>

$(S)-1$

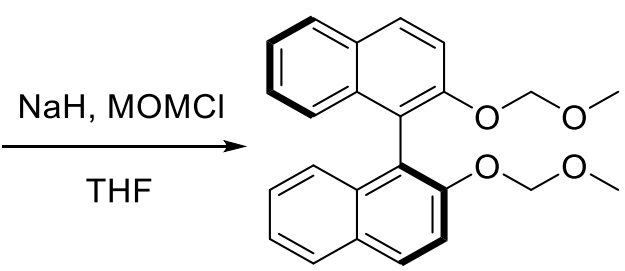

$(S)-2$

(S)-BINOL (10.0 g, $34.9 \mathrm{mmol})$ was dissolved in $100 \mathrm{~mL}$ dried THF under nitrogen atmosphere. Then $60 \% \mathrm{NaH}(4.0 \mathrm{~g}, 100 \mathrm{mmol})$ was added in portions at $0{ }^{\circ} \mathrm{C}$ in one hour. The solution was allowed to warm up to room temperature for 15 minutes. After cooling again to $0{ }^{\circ} \mathrm{C}$, chloromethyl methyl ether $(8.0 \mathrm{~mL}, 105 \mathrm{mmol})$ was added dropwise [WARNING: Chloromethyl methyl ether is carcinogenic (https://ntp.niehs.nih.gov/ntp/roc/content/profiles/bis_chloromethyl_ether.pdf). It should be carefully used under protection to prevent inhalation and dermal contact.]. The mixture was stirred at room temperature for further 3 hours. Finally, it was quenched by a saturated solution of ammonium chloride at $0{ }^{\circ} \mathrm{C}$. The aqueous phase was extracted three times with ethyl acetate. The organic solution was combined, dried over anhydrous $\mathrm{Na}_{2} \mathrm{SO}_{4}$, and then concentrated. The resulted residue was purified by silica gel column chromatography with petroleum ether/ethyl acetate $=100: 1$ as the eluent to afford the product.

$(S)-2$ (11.8 g, 90.3\% yield): ${ }^{1} \mathrm{H}$ NMR $\left(500 \mathrm{MHz}, \mathrm{CDCl}_{3}, 22{ }^{\circ} \mathrm{C}\right)$ (Figure $\left.\mathrm{S} 1\right): \delta 7.95(\mathrm{~d}, J=9.0$ $\mathrm{Hz}, 2 \mathrm{H}), 7.87(\mathrm{~d}, J=8.2 \mathrm{~Hz}, 2 \mathrm{H}), 7.57(\mathrm{~d}, J=9.0 \mathrm{~Hz}, 2 \mathrm{H}), 7.36-7.31(\mathrm{~m}, 2 \mathrm{H}), 7.24-7.19(\mathrm{~m}$, 2H), $7.15(\mathrm{~d}, J=8.5 \mathrm{~Hz}, 2 \mathrm{H}), 5.08(\mathrm{~d}, J=6.8 \mathrm{~Hz}, 2 \mathrm{H}), 4.97(\mathrm{~d}, J=6.8 \mathrm{~Hz}, 2 \mathrm{H}), 3.14(\mathrm{~s}, 6 \mathrm{H})$. ESI-TOF-MS (m/z) Calcd. for $\mathrm{C}_{24} \mathrm{H}_{22} \mathrm{NaO}_{4}[\mathrm{M}+\mathrm{Na}]^{+}:$397.1410; Found: 397.1410 . 


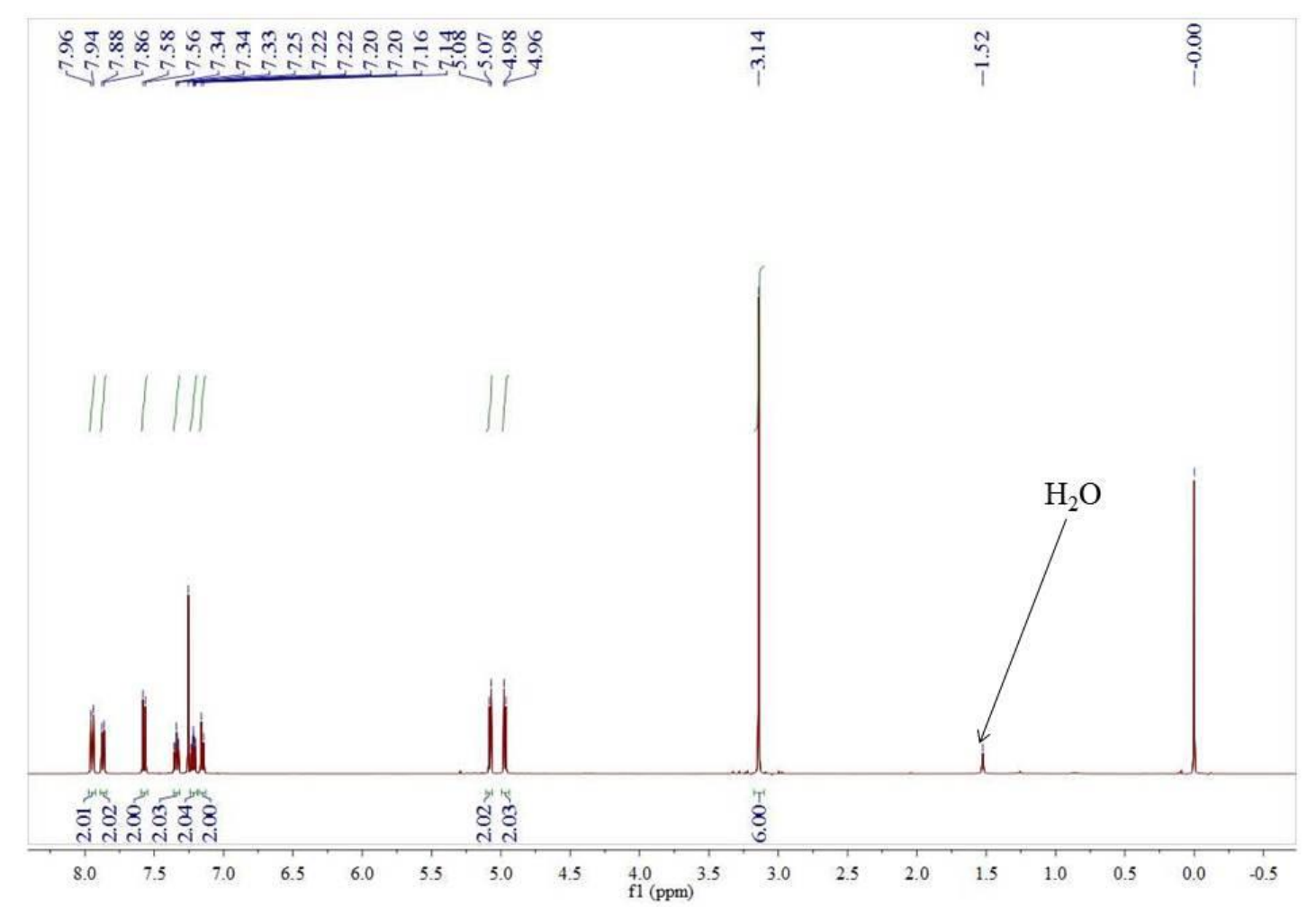

Figure S1. ${ }^{1} \mathrm{H}$ NMR $\left(500 \mathrm{MHz}, \mathrm{CDCl}_{3}, 22{ }^{\circ} \mathrm{C}\right)$ spectrum of $(S)$-2.

3. Synthesis of compound (S)-3 ${ }^{\mathrm{S4}}$

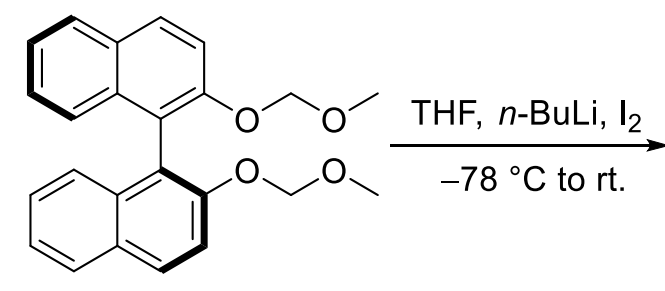

$(S)-2$<smiles></smiles>

$(S)-3$

In a dry three-necked flask, $(S)-2(6.50 \mathrm{~g}, 17.4 \mathrm{mmol})$ was dissolved in $100 \mathrm{~mL}$ dried THF under nitrogen atmosphere. $n$-Butyllithium $(20.8 \mathrm{~mL}, 2.5 \mathrm{M}$ in $n$-hexane, $52.0 \mathrm{mmol})$ was added slowly at $-78^{\circ} \mathrm{C}$. After warmed up to room temperature for about 2 hours, the mixture was cooled down to $-78{ }^{\circ} \mathrm{C}$ again. Then a solution of iodine $(13.0 \mathrm{~g}, 52.0 \mathrm{mmol})$ in $30 \mathrm{~mL}$ THF was added dropwise and the mixture was warmed up to room temperature. The reaction was further stirred at room temperature for 2 hours and then quenched with methanol. After the addition of a saturated solution of sodium thiosulfate, the mixture was extracted three times with ethyl acetate. The organic solution was combined, dried over anhydrous $\mathrm{Na}_{2} \mathrm{SO}_{4}$, and then concentrated. The resulted residue was purified by silica gel column chromatography with petroleum ether/ethyl acetate $=200: 1$ as the eluent to afford the product. 
(S)-3 (7.80 g, 71.7\% yield): ${ }^{1} \mathrm{H}$ NMR (500 MHz, $\left.\mathrm{CDCl}_{3}, 22{ }^{\circ} \mathrm{C}\right)$ (Figure S2): $\delta 8.54(\mathrm{~s}, 2 \mathrm{H})$, $7.78(\mathrm{~d}, J=8.2 \mathrm{~Hz}, 2 \mathrm{H}), 7.45-7.40(\mathrm{~m}, 2 \mathrm{H}), 7.32-7.27(\mathrm{~m}, 2 \mathrm{H}), 7.17(\mathrm{~d}, J=8.5 \mathrm{~Hz}, 2 \mathrm{H}), 4.81$ $(\mathrm{d}, J=5.7 \mathrm{~Hz}, 2 \mathrm{H}), 4.69(\mathrm{~d}, J=5.7 \mathrm{~Hz}, 2 \mathrm{H}), 2.60(\mathrm{~s}, 6 \mathrm{H})$. ESI-TOF-MS $(\mathrm{m} / \mathrm{z})$ Calcd. for $\mathrm{C}_{24} \mathrm{H}_{20} \mathrm{I}_{2} \mathrm{NaO}_{4}[\mathrm{M}+\mathrm{Na}]^{+}:$648.9343; Found: 648.9332 .

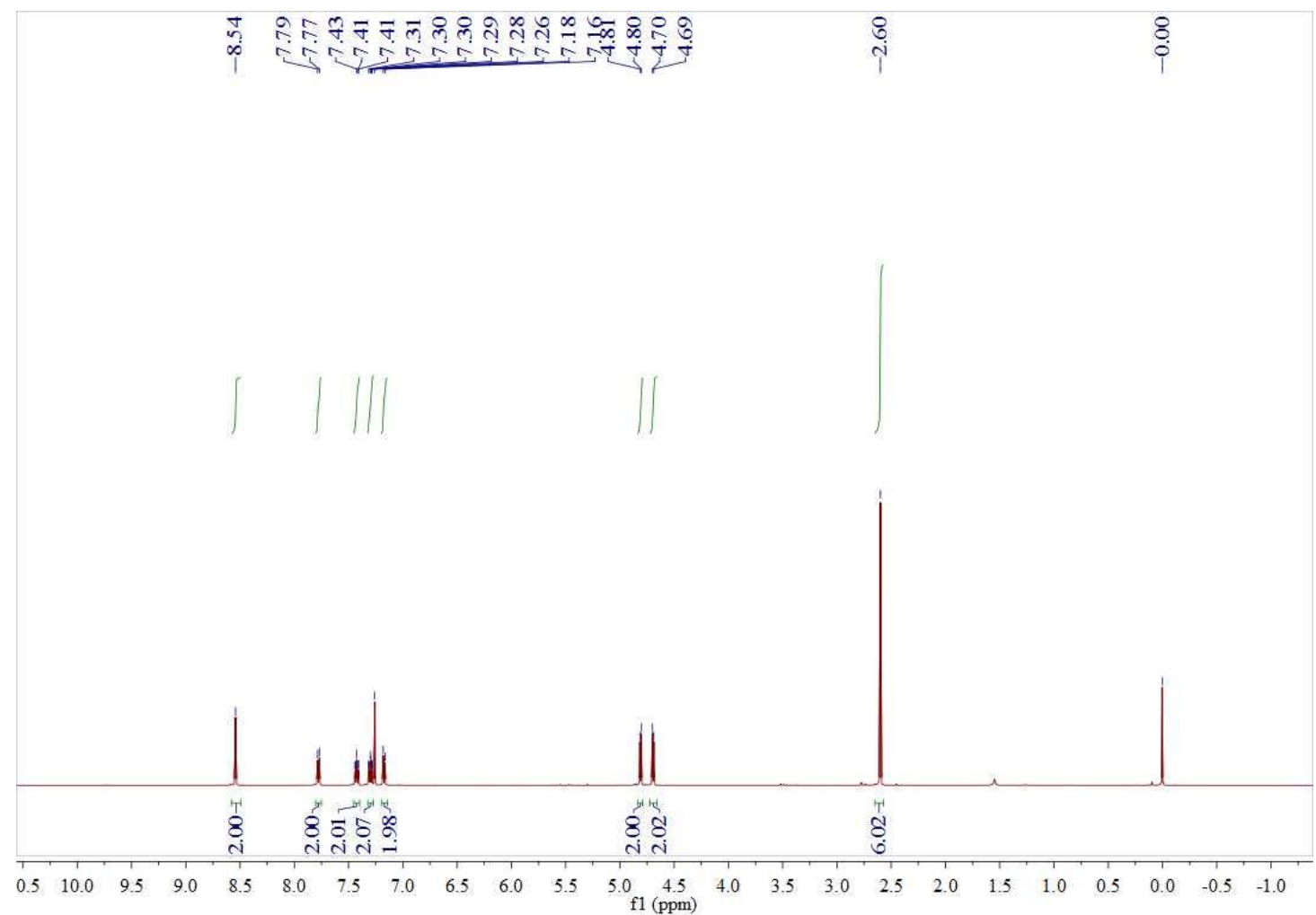

Figure S2. ${ }^{1} \mathrm{H}$ NMR $\left(500 \mathrm{MHz}, \mathrm{CDCl}_{3}, 22{ }^{\circ} \mathrm{C}\right)$ spectrum of $(S)-\mathbf{3}$.

\section{Synthesis of compound (S)-4 ${ }^{\mathrm{S} 4}$}<smiles>COCOc1c(I)cc2ccccc2c1-c1c(OCOC)c(I)cc2ccccc12</smiles>

$(S)-3$<smiles>COCOc1c(-c2ccncc2)cc2ccccc2c1-c1c(OCOC)c(-c2ccncc2)cc2ccccc12</smiles>

(S)-4

(S)-3 (400 mg, $0.64 \mathrm{mmol}$ ), 4-pyridylboronic acid pinacol ester (301 mg, $1.47 \mathrm{mmol}$ ), potassium phosphate $(814 \mathrm{mg}, 3.8 \mathrm{mmol})$, tris(dibenzylideneacetone)dipalladium (13.2 $\mathrm{mg}$, $0.013 \mathrm{mmol}$ ), tricyclohexylphosphine ( $8.6 \mathrm{mg}, 0.030 \mathrm{mmol}), 5.3 \mathrm{~mL}$ of 1,4-dioxane and 1.5 $\mathrm{mL} \mathrm{H}_{2} \mathrm{O}$ were mixed and refluxed for 18 hours under $\mathrm{N}_{2}$. After cooling to room temperature, 
the reaction was firstly quenched by a saturated solution of EDTA, then extracted with $\mathrm{CH}_{2} \mathrm{Cl}_{2}$ and washed twice with saturated brine. The organic solution was dried over anhydrous $\mathrm{Na}_{2} \mathrm{SO}_{4}$ and then concentrated. The product $(S)-\mathbf{4}$ was obtained by silica gel column chromatography (petroleum ether/ethyl acetate/triethylamine $=1: 1: 0.05$ ).

(S)-4 (300 mg, 89.0\% yield): $\mathrm{Mp} 131.1-133.1{ }^{\circ} \mathrm{C} .{ }^{1} \mathrm{H} \mathrm{NMR}\left(500 \mathrm{MHz}, \mathrm{CDCl}_{3}, 22{ }^{\circ} \mathrm{C}\right)$ (Figure S3): $\delta 8.72(\mathrm{~d}, J=6.0 \mathrm{~Hz}, 4 \mathrm{H}), 8.00(\mathrm{~s}, 2 \mathrm{H}), 7.93(\mathrm{~d}, J=8.1 \mathrm{~Hz}, 2 \mathrm{H}), 7.72(\mathrm{~d}, J=6.1 \mathrm{~Hz}, 4 \mathrm{H})$, 7.48-7.45 (m, 2H), 7.34-7.33 (m, 2H), 7.28 (d, $J=8.4 \mathrm{~Hz}, 2 \mathrm{H}), 4.41(\mathrm{~d}, J=6.0 \mathrm{~Hz}, 2 \mathrm{H}), 4.36$ $(\mathrm{d}, J=6.0 \mathrm{~Hz}, 2 \mathrm{H}), 2.40(\mathrm{~s}, 6 \mathrm{H}) \cdot{ }^{13} \mathrm{C}$ NMR $\left(125 \mathrm{MHz}, d_{6}\right.$-DMSO, $22{ }^{\circ} \mathrm{C}$ ) (Figure $\left.\mathrm{S} 4\right) \delta$ $151.11,149.72$, 147.08, 134.09, 132.80, 130.90, 130.71, 128.24, 127.28, 126.55, 126.33, 125.74, 124.47, 99.06, 56.08. ESI-TOF-MS ( $\mathrm{m} / \mathrm{z})$ Calcd. for $\mathrm{C}_{34} \mathrm{H}_{29} \mathrm{~N}_{2} \mathrm{O}_{4}[\mathrm{M}+\mathrm{H}]^{+}:$529.2122; Found: 529.2140.

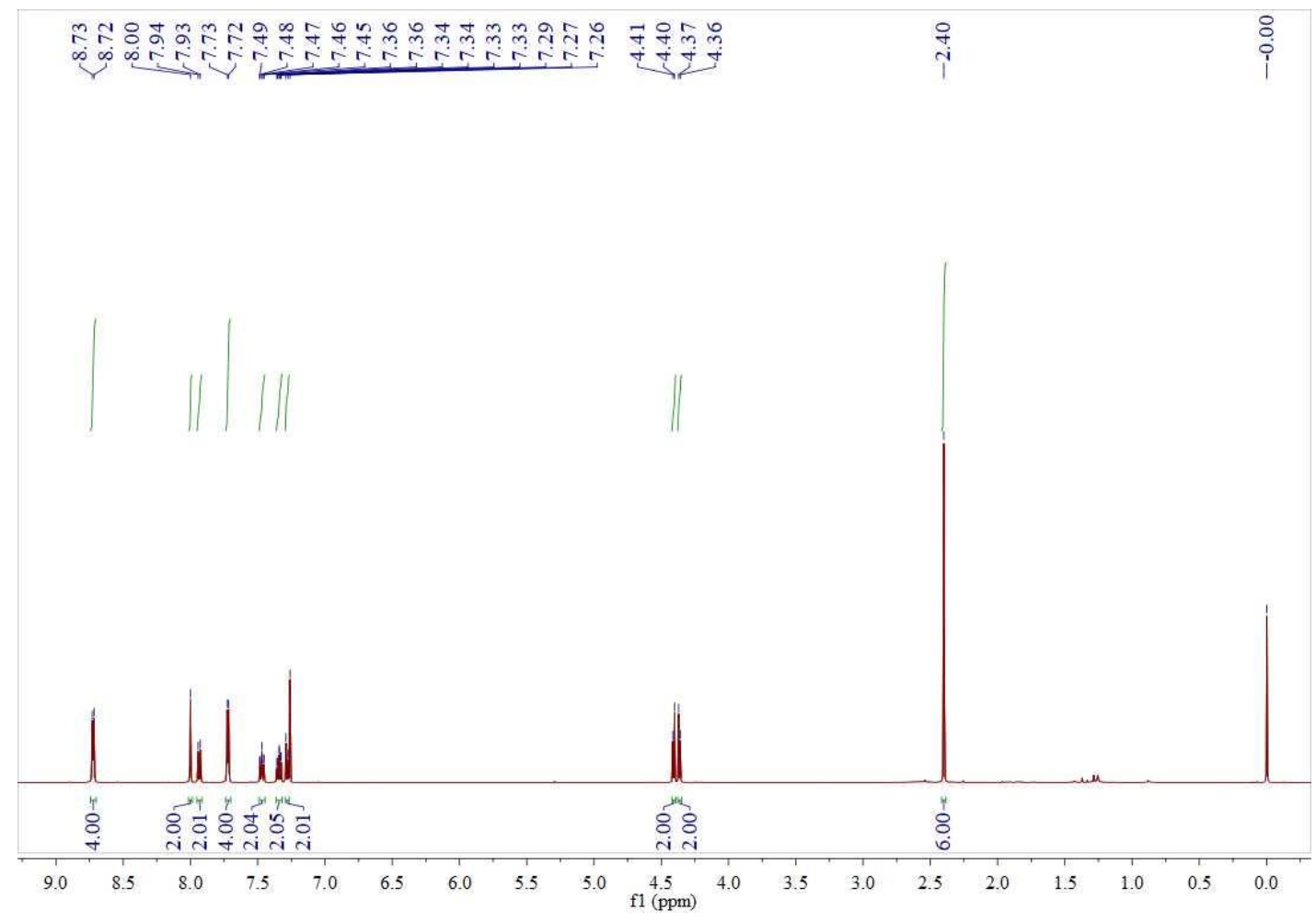

Figure S3. ${ }^{1} \mathrm{H}$ NMR $\left(500 \mathrm{MHz}, \mathrm{CDCl}_{3}, 22{ }^{\circ} \mathrm{C}\right)$ spectrum of $(S)-\mathbf{4}$. 


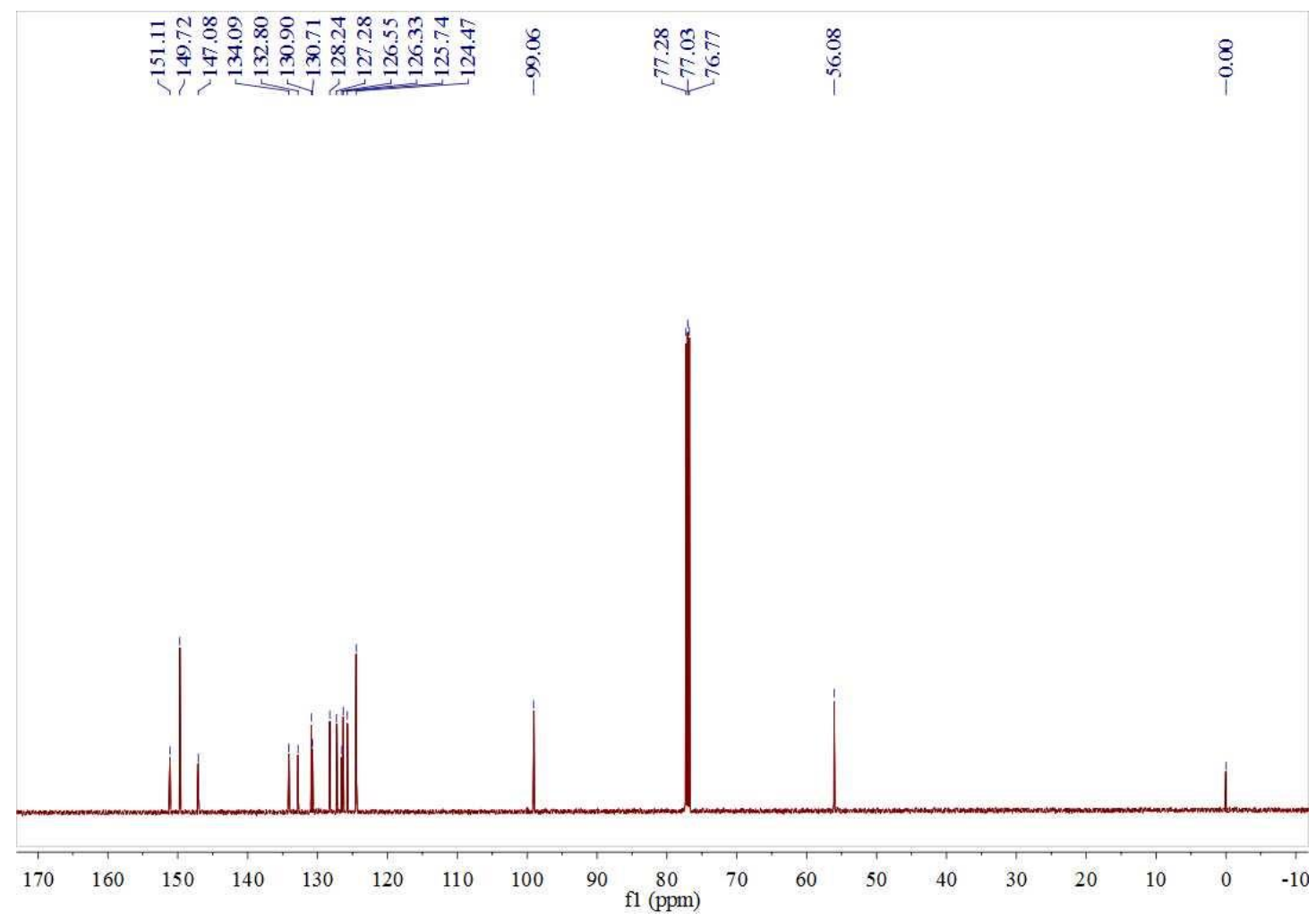

Figure S4. ${ }^{13} \mathrm{C}$ NMR $\left(500 \mathrm{MHz}, \mathrm{CDCl}_{3}, 22{ }^{\circ} \mathrm{C}\right)$ spectrum of $(S)-4$.

\section{Synthesis of compound (S)-5}<smiles>COCOc1c(-c2ccncc2)cc2ccccc2c1-c1c(OCOC)c(-c2ccncc2)cc2ccccc12</smiles>

$(S)-4$<smiles>Oc1c(-c2ccncc2)cc2ccccc2c1-c1c(O)c(-c2ccncc2)cc2ccccc12</smiles>

(S)-5

A mixture of $(S)-4(400 \mathrm{mg}, 0.76 \mathrm{mmoL})$, concentrated $\mathrm{HCl}(4 \mathrm{~mL})$ and $\mathrm{THF}(20 \mathrm{~mL})$ were stirred at room temperature for 18 hours under nitrogen atmosphere. After the removal of THF by rotary evaporation, $10 \mathrm{~mL}$ water was added and $\mathrm{pH}$ of the solution was adjusted to neutral by the addition of a diluted $\mathrm{NaOH}$ solution. Finally, $(S)-\mathbf{5}$ was collected by filtration and washed twice with absolute ethanol.

$(S)-5\left(310 \mathrm{mg}, 90.0 \%\right.$ yield): $\mathrm{Mp}>400{ }^{\circ} \mathrm{C} .{ }^{1} \mathrm{H}$ NMR $\left(500 \mathrm{MHz}, d_{6}\right.$-DMSO, $\left.22{ }^{\circ} \mathrm{C}\right)($ Figure S5): $\delta 8.67(\mathrm{~s}, 6 \mathrm{H}), 8.10(\mathrm{~s}, 2 \mathrm{H}), 7.99(\mathrm{~d}, J=8.0 \mathrm{~Hz}, 2 \mathrm{H}), 7.75(\mathrm{~d}, J=5.3 \mathrm{~Hz}, 4 \mathrm{H}), 7.33(\mathrm{t}, J=$ 
$7.3 \mathrm{~Hz}, 2 \mathrm{H}), 7.26(\mathrm{t}, J=7.4 \mathrm{~Hz}, 2 \mathrm{H}), 6.92(\mathrm{~d}, J=8.4 \mathrm{~Hz}, 2 \mathrm{H}) .{ }^{13} \mathrm{C}$ NMR $(125 \mathrm{MHz}$, $d_{6}$-DMSO, $22{ }^{\circ} \mathrm{C}$ ) (Figure S7): $\delta$ 151.67, 149.70, 147.03, 134.57, 130.99, 129.47, 128.99, 127.41, 124.95, 124.44, 123.75, 115.47. ESI-TOF-MS (m/z) Calcd. for $\mathrm{C}_{30} \mathrm{H}_{21} \mathrm{~N}_{2} \mathrm{O}_{2}[\mathrm{M}+\mathrm{H}]^{+}$: 441.1598; Found: 441.1634.

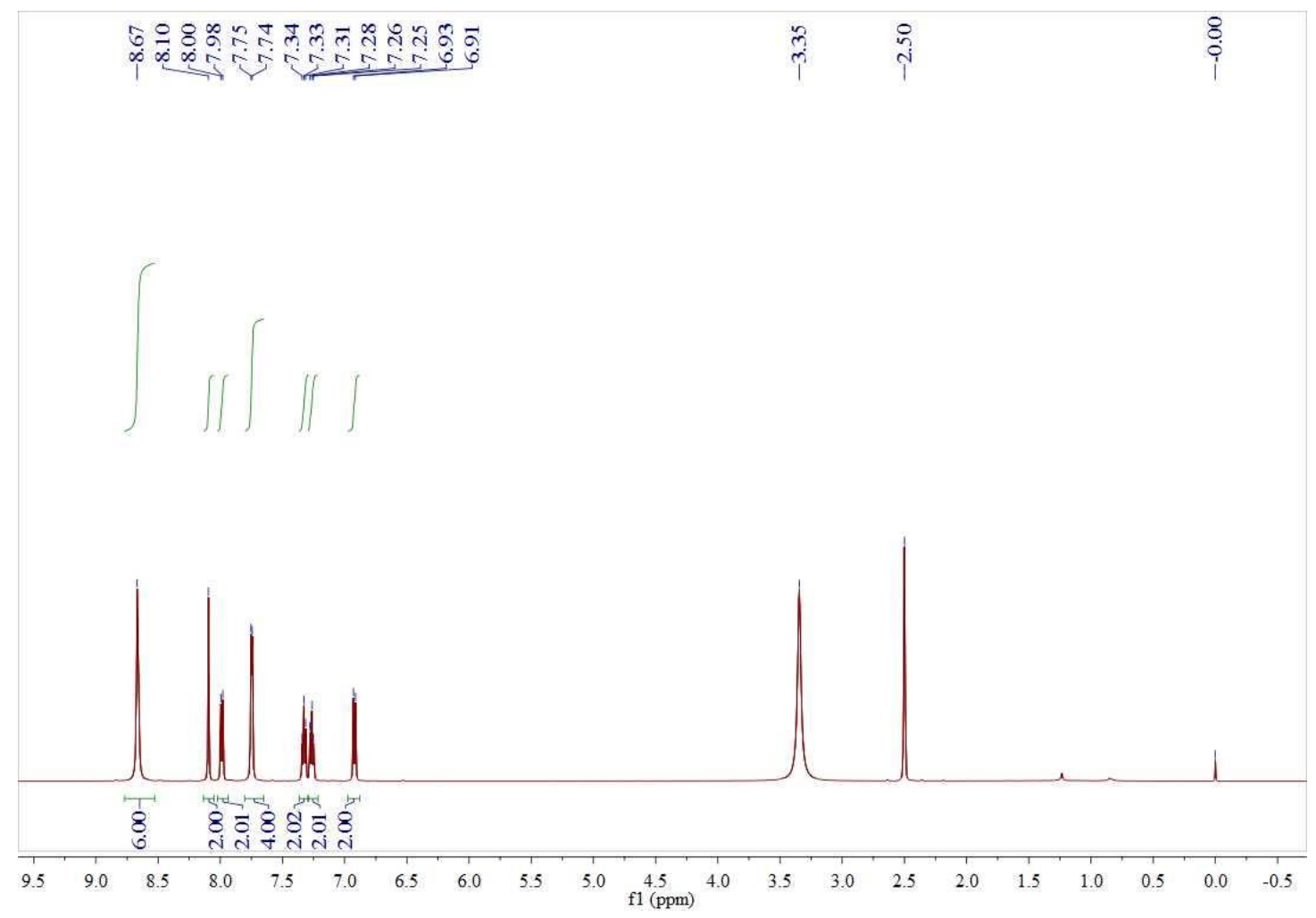

Figure S5. ${ }^{1} \mathrm{H}$ NMR $\left(500 \mathrm{MHz}, d_{6}\right.$-DMSO, $\left.22{ }^{\circ} \mathrm{C}\right)$ spectrum of $(S)-\mathbf{5}$. 


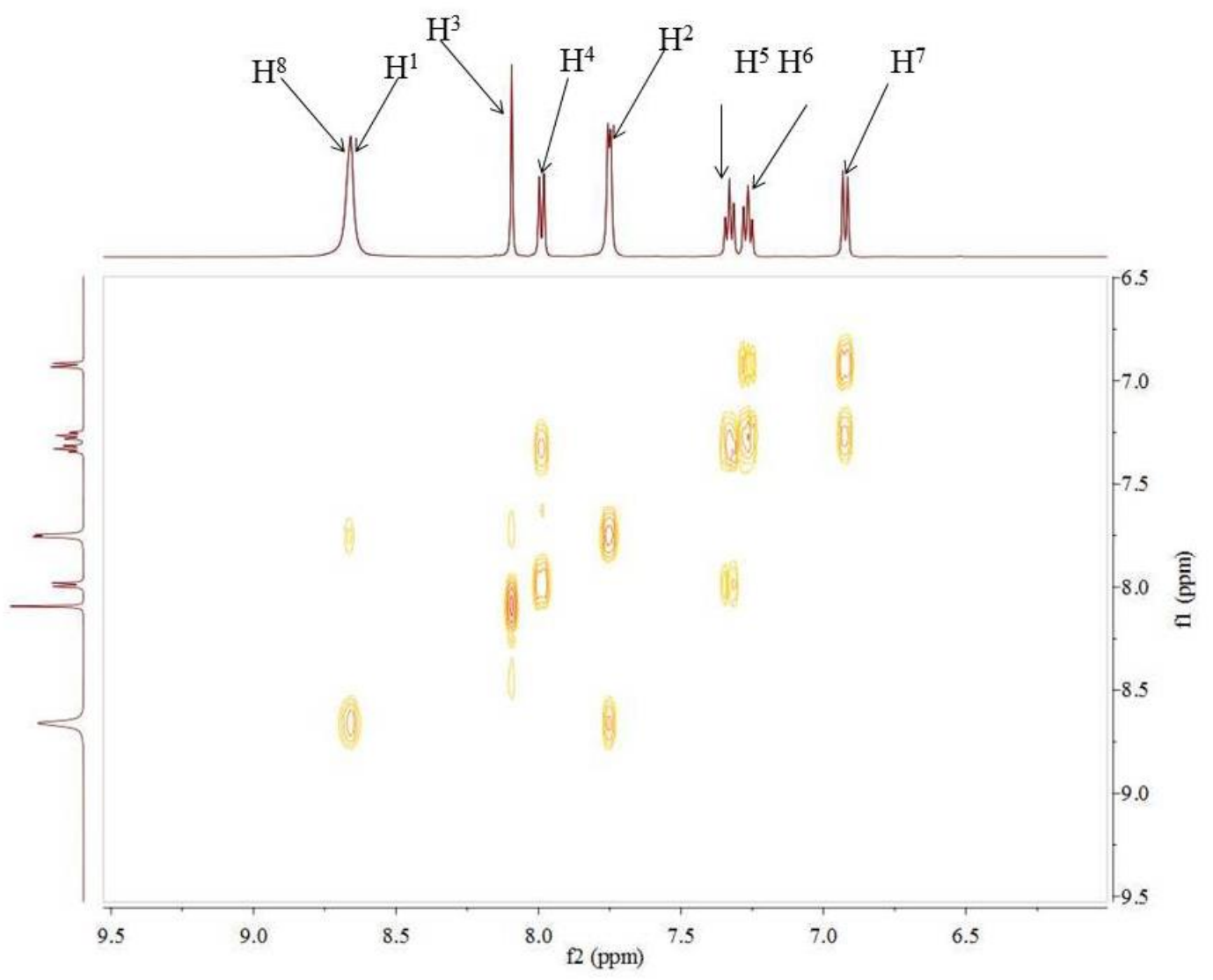

Figure S6. ${ }^{1} \mathrm{H}-{ }^{1} \mathrm{H}$ COSY spectrum $\left(500 \mathrm{MHz}, d_{6}\right.$-DMSO, $\left.22{ }^{\circ} \mathrm{C}\right)$ of $(S)-5$.

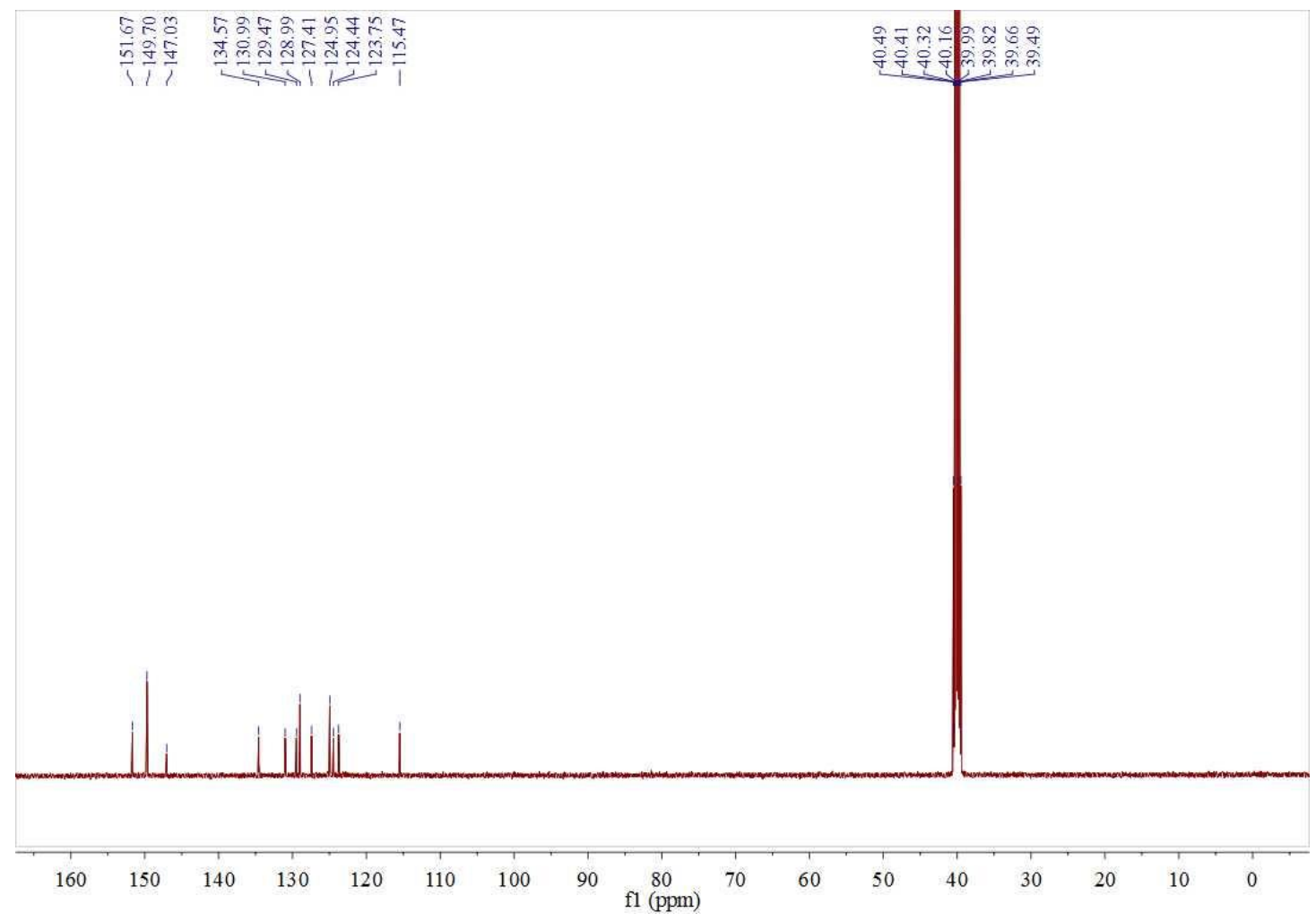

Figure S7. ${ }^{13} \mathrm{C}$ NMR $\left(125 \mathrm{MHz}, d_{6}\right.$-DMSO, $\left.22{ }^{\circ} \mathrm{C}\right)$ spectrum of $(S)-5$. 


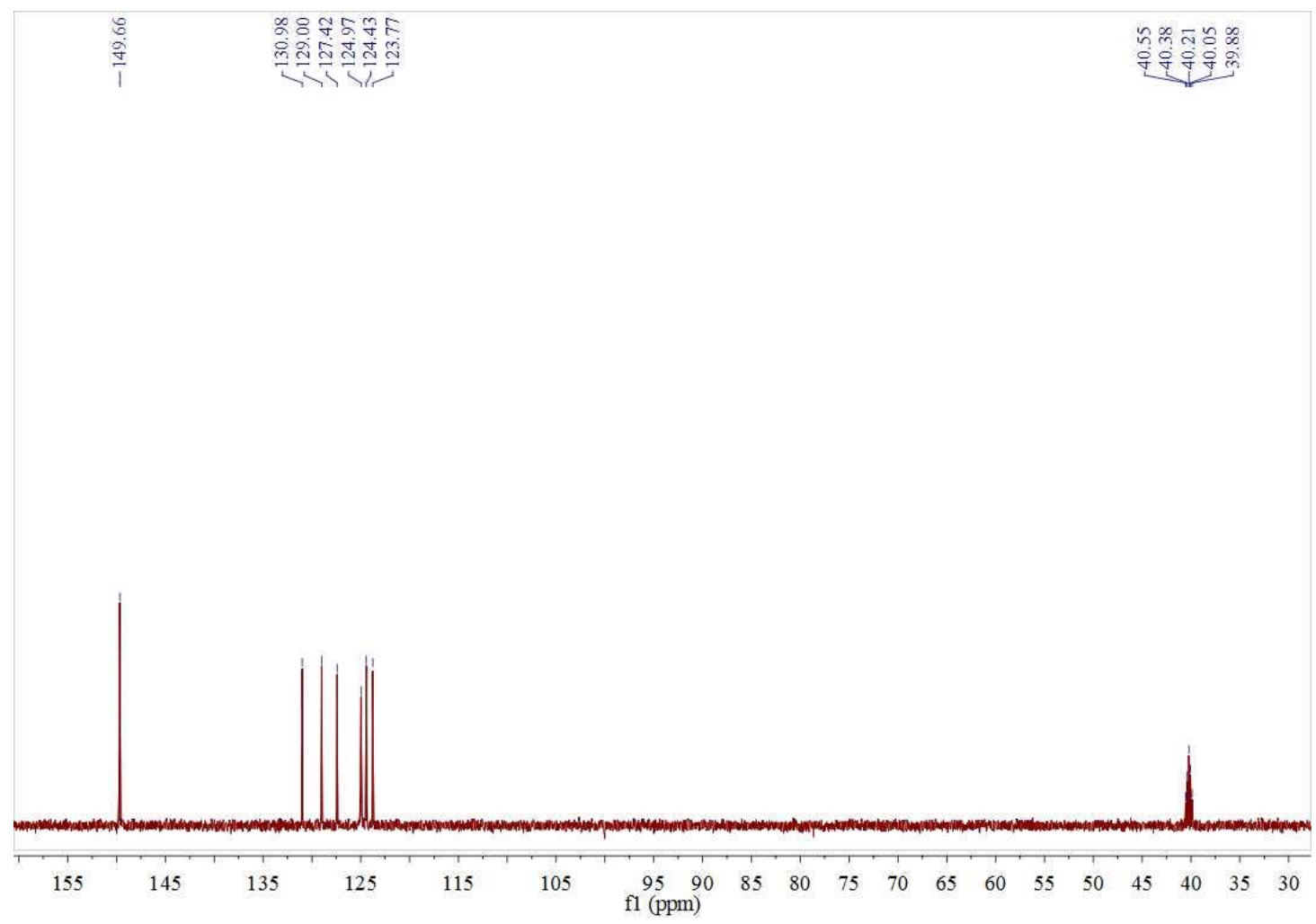

Figure S8. ${ }^{13} \mathrm{C}$ Dept $90^{\circ} \mathrm{NMR}\left(125 \mathrm{MHz}, d_{6}\right.$-DMSO, $\left.22{ }^{\circ} \mathrm{C}\right)$ spectrum of $(S)-5$.

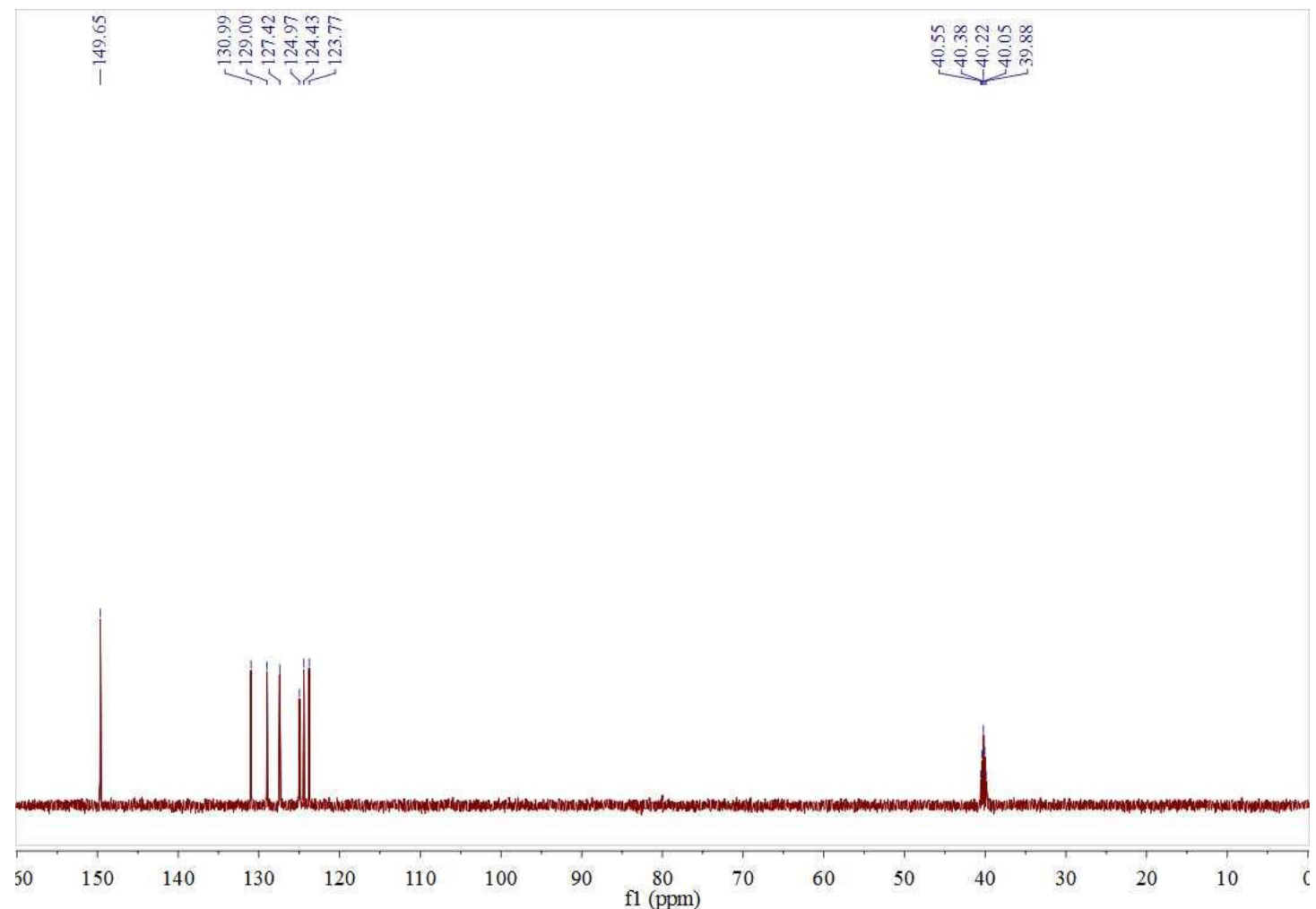

Figure S9. ${ }^{13} \mathrm{C}$ Dept $135^{\circ} \mathrm{NMR}\left(125 \mathrm{MHz}, d_{6}\right.$-DMSO, $\left.22{ }^{\circ} \mathrm{C}\right)$ spectrum of $(S)-5$. 

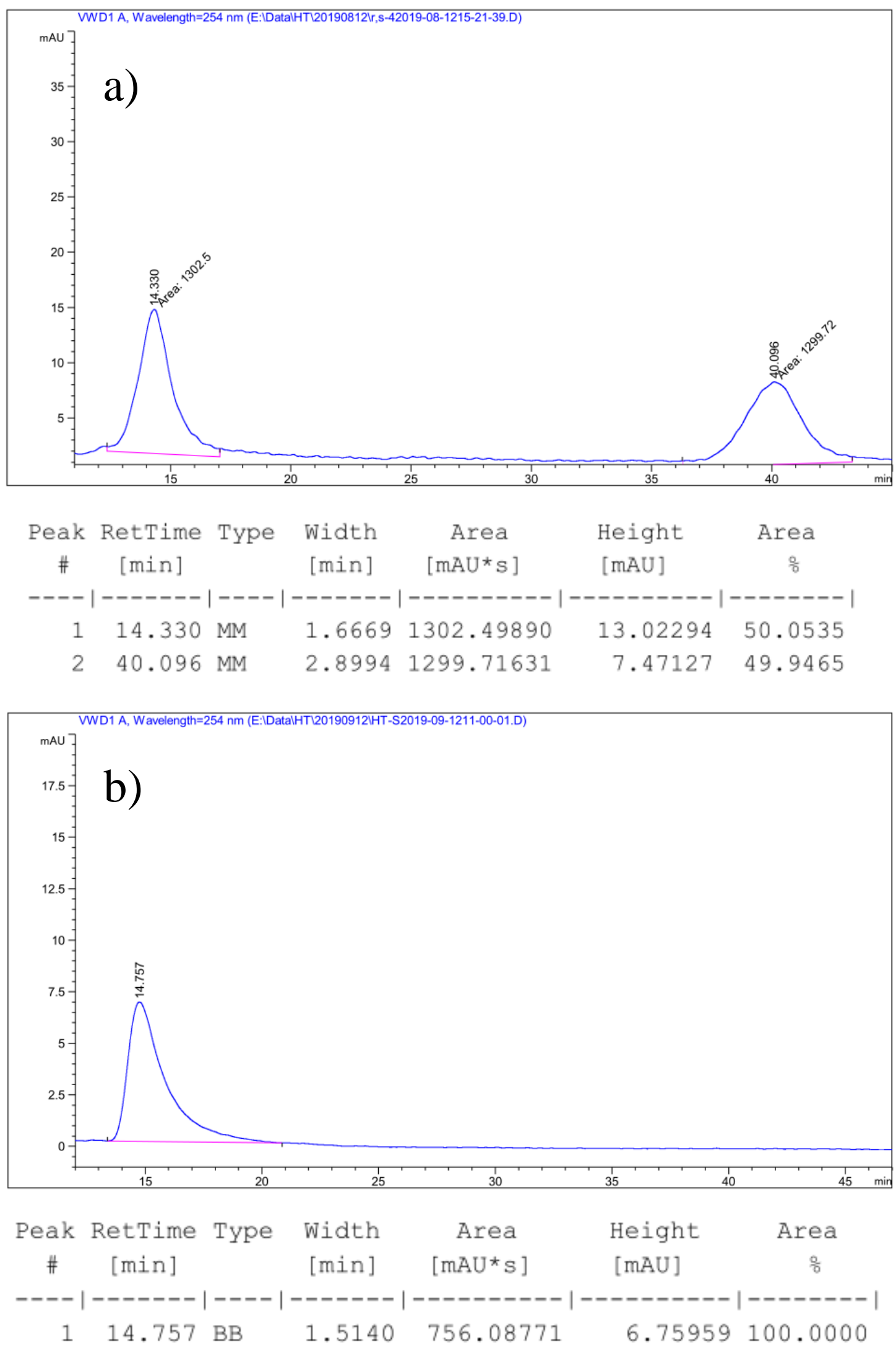

Figure S10. Chiral HPLC analysis of a) racemic 5, and b) (S)-5 (Chiralpak AD, hexane/2-propanol 80/20, $1.0 \mathrm{~mL} / \mathrm{min}, \lambda=254 \mathrm{~nm}$ ). 


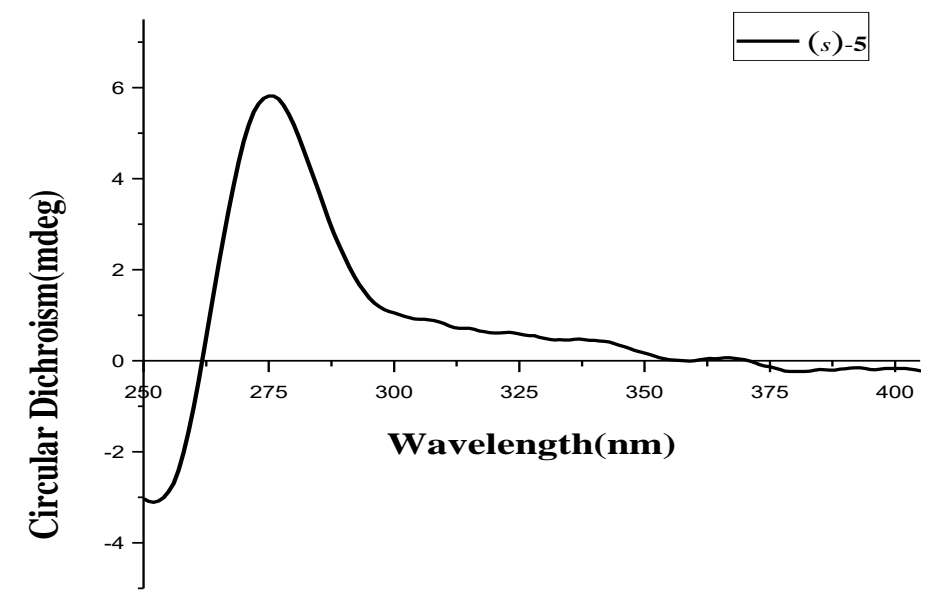

Figure S11. CD spectrum of $(S)-5$ in DMSO.

6. Self-assembly of chiral metalla-triangle (S)-7a

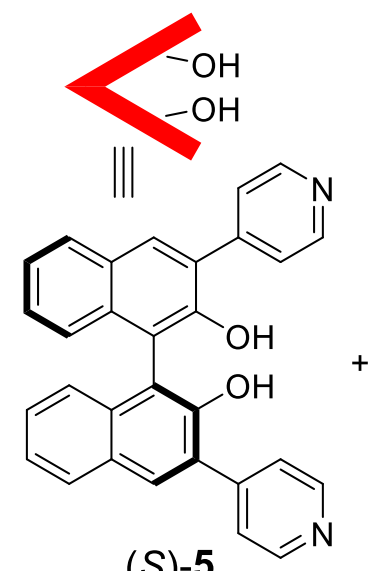

(S)-5

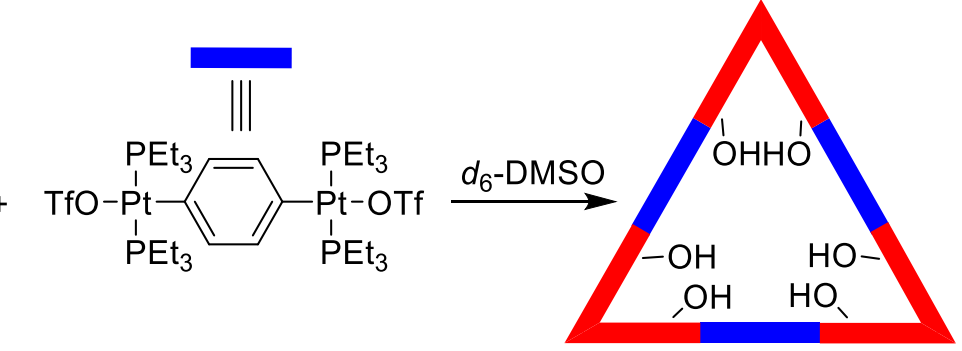

$6 a$
$(S)-7 a$

$(S)$-5 $(8.00 \mathrm{mg}, 0.018 \mathrm{mmol})$ and $\mathbf{6 a}(22.45 \mathrm{mg}, 0.018 \mathrm{mmol})$ were placed in a 2-dram vial, followed by addition of $d_{6}$-DMSO. After stirring overnight at $50{ }^{\circ} \mathrm{C}$, the assembly $(S)$-7a was obtained.

(S)-7a (91.39 mg, $100 \%$ yield): ${ }^{1} \mathrm{H}$ NMR (500 MHz, $d_{6}$-DMSO, $22{ }^{\circ} \mathrm{C}$ ) (Figure S12): $\delta 8.97$ (s, 2H), $8.85(\mathrm{~s}, 4 \mathrm{H}), 8.33(\mathrm{~s}, 2 \mathrm{H}), 8.10(\mathrm{~s}, 6 \mathrm{H}), 7.35(\mathrm{dd}, J=21.3,14.2 \mathrm{~Hz}, 4 \mathrm{H}), 7.03-6.97(\mathrm{~m}$, $6 \mathrm{H}), 1.39(\mathrm{~s}, 24 \mathrm{H}), 1.10-1.07(\mathrm{~m}, 36 \mathrm{H}) .{ }^{31} \mathrm{P}\left\{{ }^{1} \mathrm{H}\right\}$ NMR (202 MHz, $d_{6}$-DMSO, $22{ }^{\circ} \mathrm{C}$ ) (Figure S14): $\delta 13.05\left(\mathrm{~s},{ }^{195} \mathrm{Pt}\right.$ satellites, $\left.J_{\mathrm{Pt}-\mathrm{P}}=1361 \mathrm{~Hz}\right)$. ESI-TOF-MS $(\mathrm{m} / \mathrm{z})$ (Figure S15): Calcd. for $[(S)-7 \mathbf{a}-4 \text { HOTf }-2 \text { OTf }+1 \mathrm{~K}]^{3+}:$ 1390.7863; Found: 1390.7892; error: $2.1 \mathrm{ppm}$. 


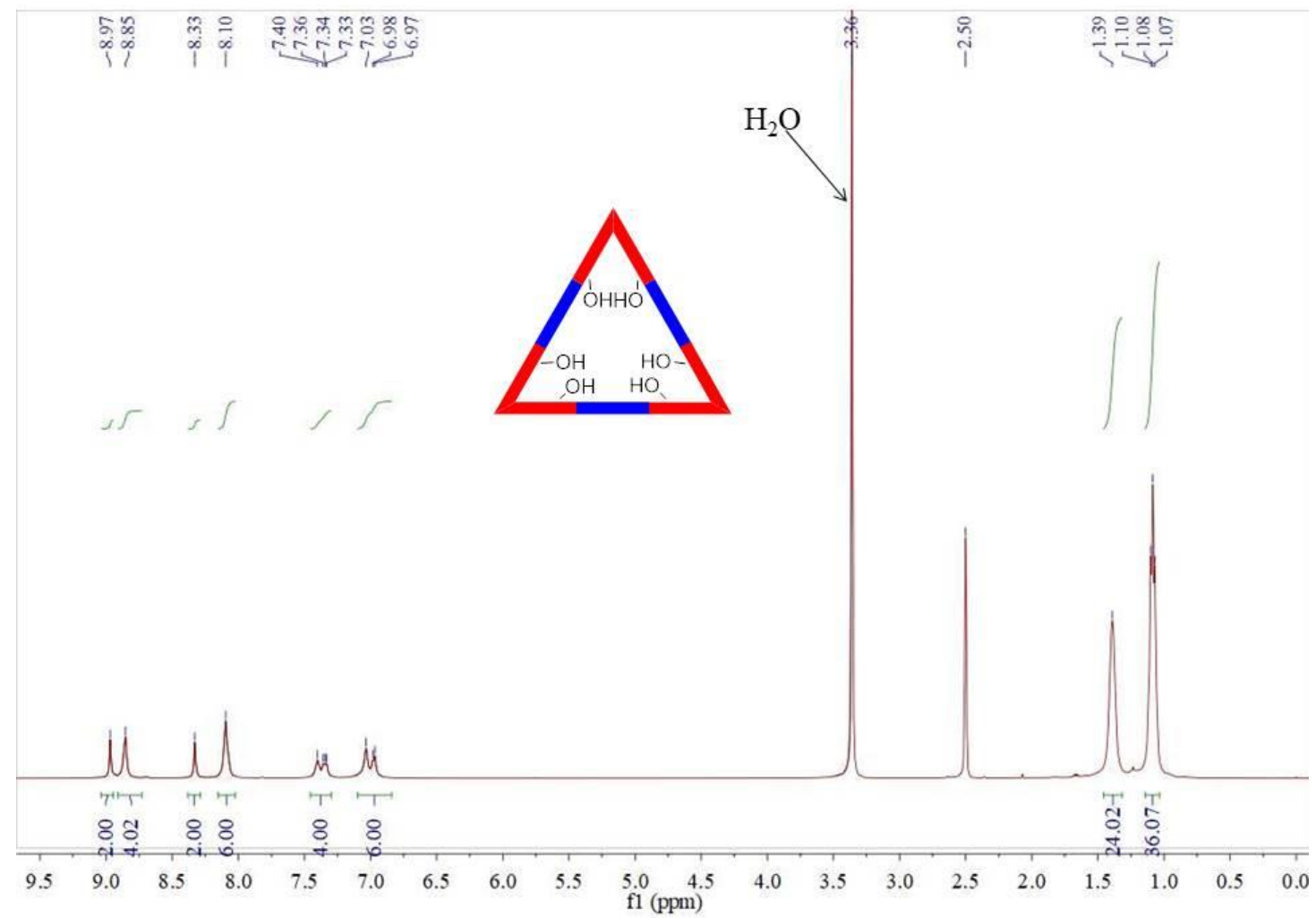

Figure S12. ${ }^{1} \mathrm{H}$ NMR $\left(500 \mathrm{MHz}, d_{6}\right.$-DMSO, $\left.22{ }^{\circ} \mathrm{C}\right)$ spectrum of $(S)-7 \mathbf{a}$.

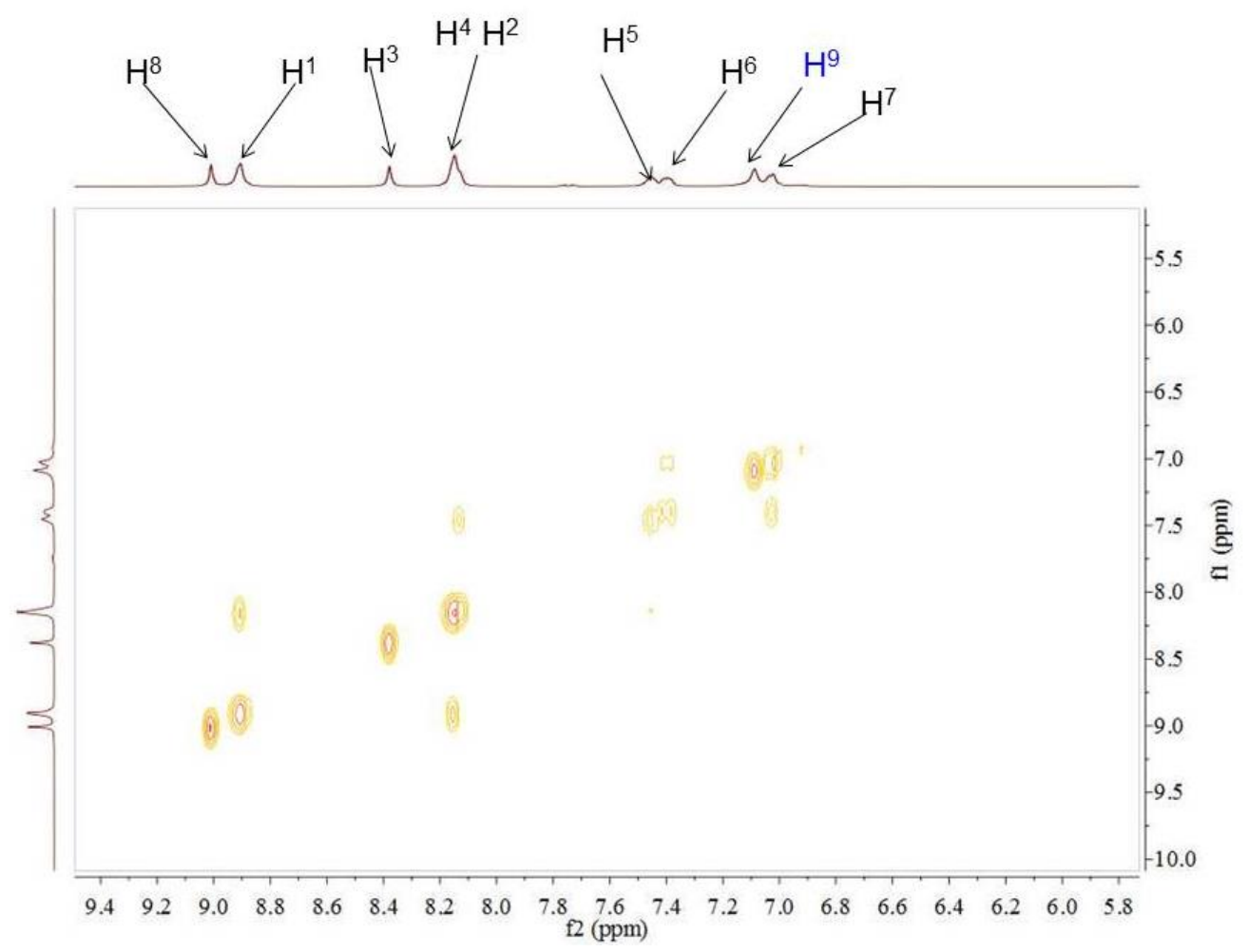

Figure S13. ${ }^{1} \mathrm{H}-{ }^{1} \mathrm{H}$ COSY $\left(500 \mathrm{MHz}, d_{6}\right.$-DMSO, $\left.22{ }^{\circ} \mathrm{C}\right)$ spectrum of $(S)-7 \mathbf{a}$. 


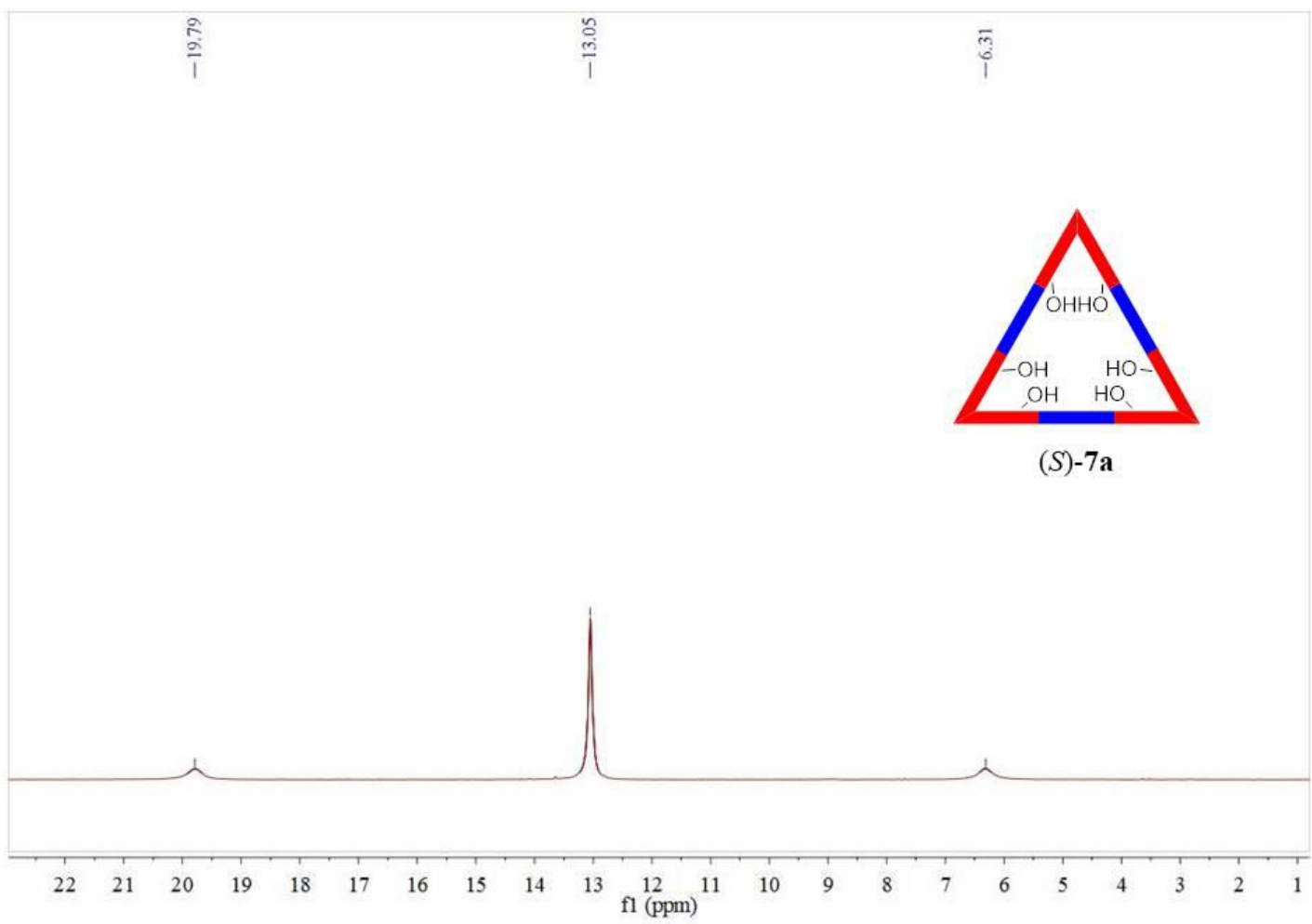

Figure S14. ${ }^{31} \mathrm{P}\left\{{ }^{1} \mathrm{H}\right\}$ NMR $\left(202 \mathrm{MHz}, d_{6}\right.$-DMSO, $\left.22{ }^{\circ} \mathrm{C}\right)$ spectrum of $(S)-7 \mathbf{a}$. 

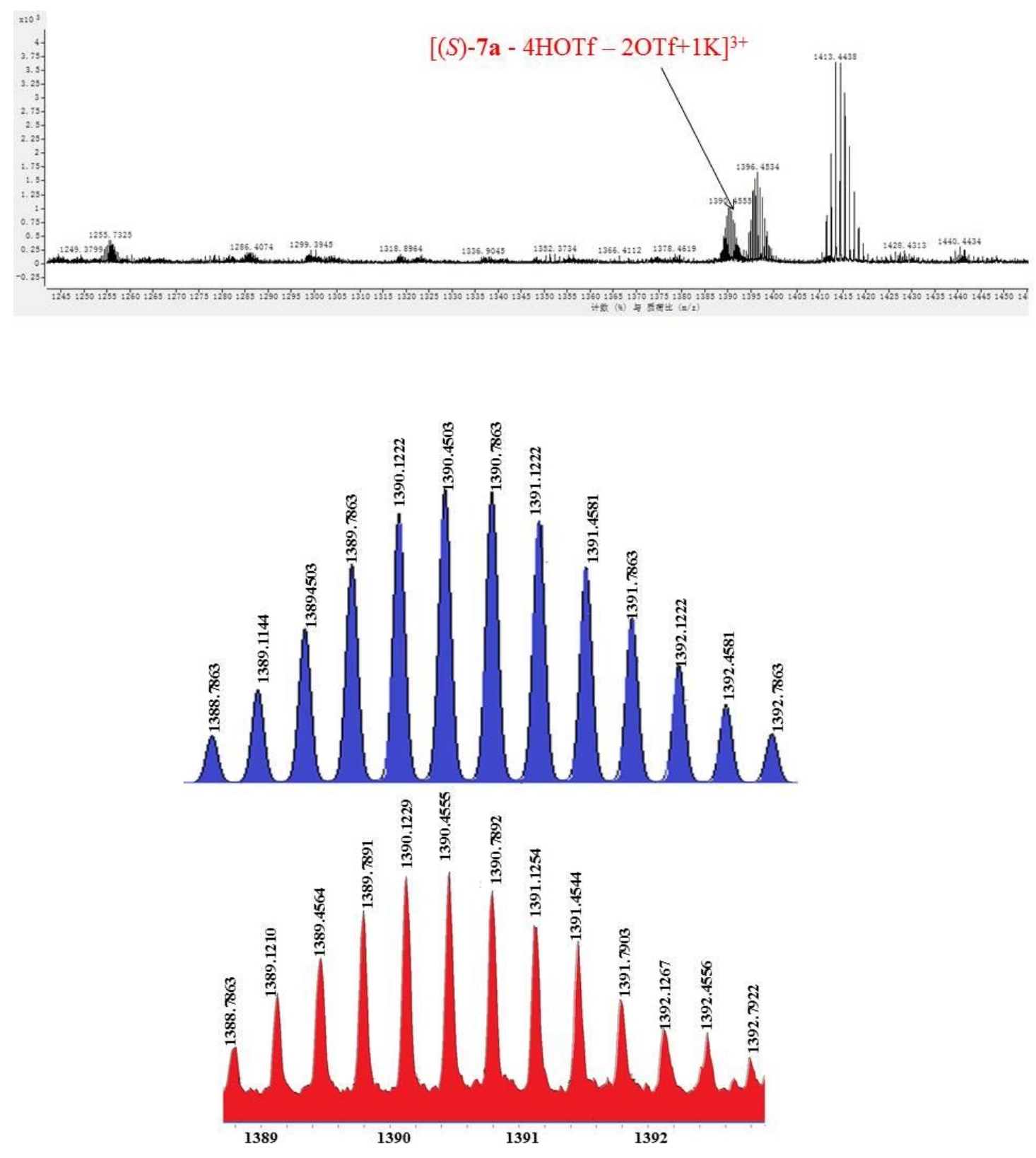

Figure S15. ESI-MS spectrum of $[(S)-7 \mathbf{a}-4 \mathrm{HOTf}-2 \mathrm{OTf}+1 \mathrm{~K}]^{3+}$ at $\mathrm{m} / z=1390.7892($ red) and its simulated spectra (blue). 


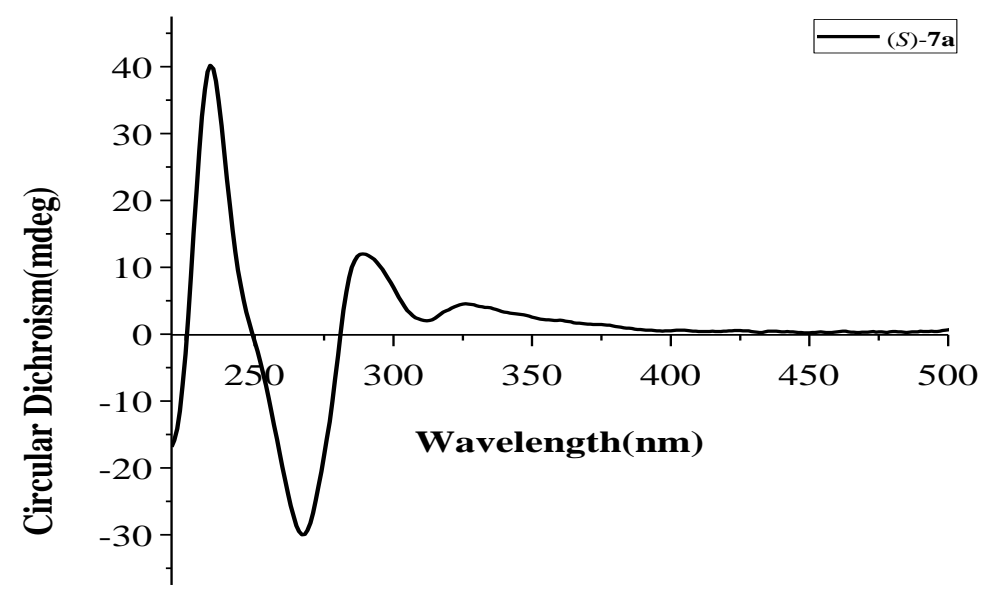

Figure S16. CD spectrum of $(S)-7 \mathbf{a}$ in $\mathrm{CH}_{2} \mathrm{Cl}_{2}$.

7. Self-assembly of chiral metalla-triangle (S)-7b

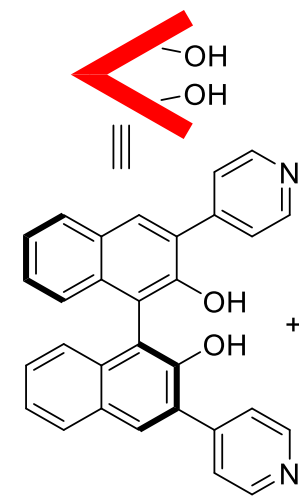

$(S)-5$

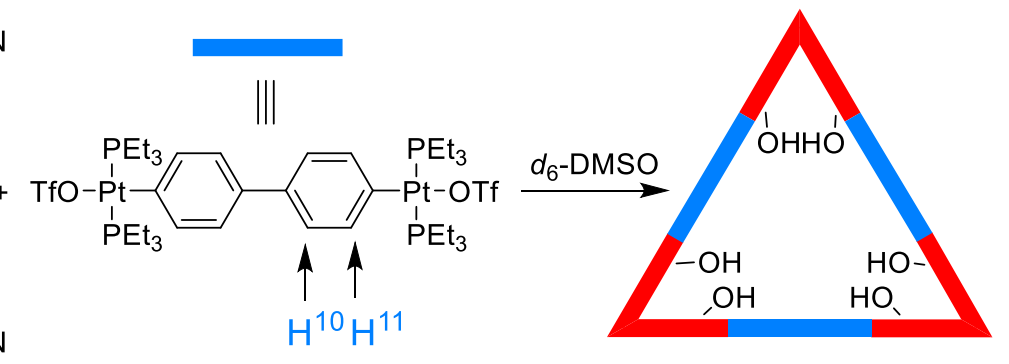

$6 b$
$(S)-7 \mathbf{b}$

(S)-5 (8.00 mg, $0.018 \mathrm{mmol})$ and $\mathbf{6 b}(23.85 \mathrm{mg}, 0.018 \mathrm{mmol})$ were placed in a 2-dram vial, followed by addition of $d_{6}$-DMSO. After stirring overnight at $50{ }^{\circ} \mathrm{C}$, the assembly $(S)-7 \mathbf{b}$ was obtained.

$(S)-7 \mathbf{b}\left(95.77 \mathrm{mg}, 100 \%\right.$ yield): ${ }^{1} \mathrm{H}$ NMR (500 MHz, $d_{6}$-DMSO, $22{ }^{\circ} \mathrm{C}$ ) (Figure S17): $\delta 8.98$ (s, 2H), $8.89(\mathrm{~s}, 4 \mathrm{H}), 8.35(\mathrm{~s}, 2 \mathrm{H}), 8.12-8.08(\mathrm{~m}, 6 \mathrm{H}), 7.49-7.30(\mathrm{~m}, 12 \mathrm{H}), 6.99(\mathrm{~d}, J=7.1 \mathrm{~Hz}$, 2H), 1.39 (s, 24H), 1.13-1.10 (m, 36H). ${ }^{31} \mathrm{P}\left\{{ }^{1} \mathrm{H}\right\}$ NMR (202 MHz, $d_{6}$-DMSO, $22{ }^{\circ} \mathrm{C}$ ) (Figure S19): $\delta 13.73$ (s, ${ }^{195} \mathrm{Pt}$ satellites, $J_{\mathrm{Pt}-\mathrm{P}}=1337 \mathrm{~Hz}$ ). ESI-TOF-MS $(\mathrm{m} / \mathrm{z})$ (Figure S20): Calcd. for $[(S)-7 \mathbf{b}-2 \mathrm{HOTf}-2 \mathrm{OTf}+1 \mathrm{~K}]^{3+}: 1566.4548$; Found: 1566.4625; error: 4.9 ppm. 


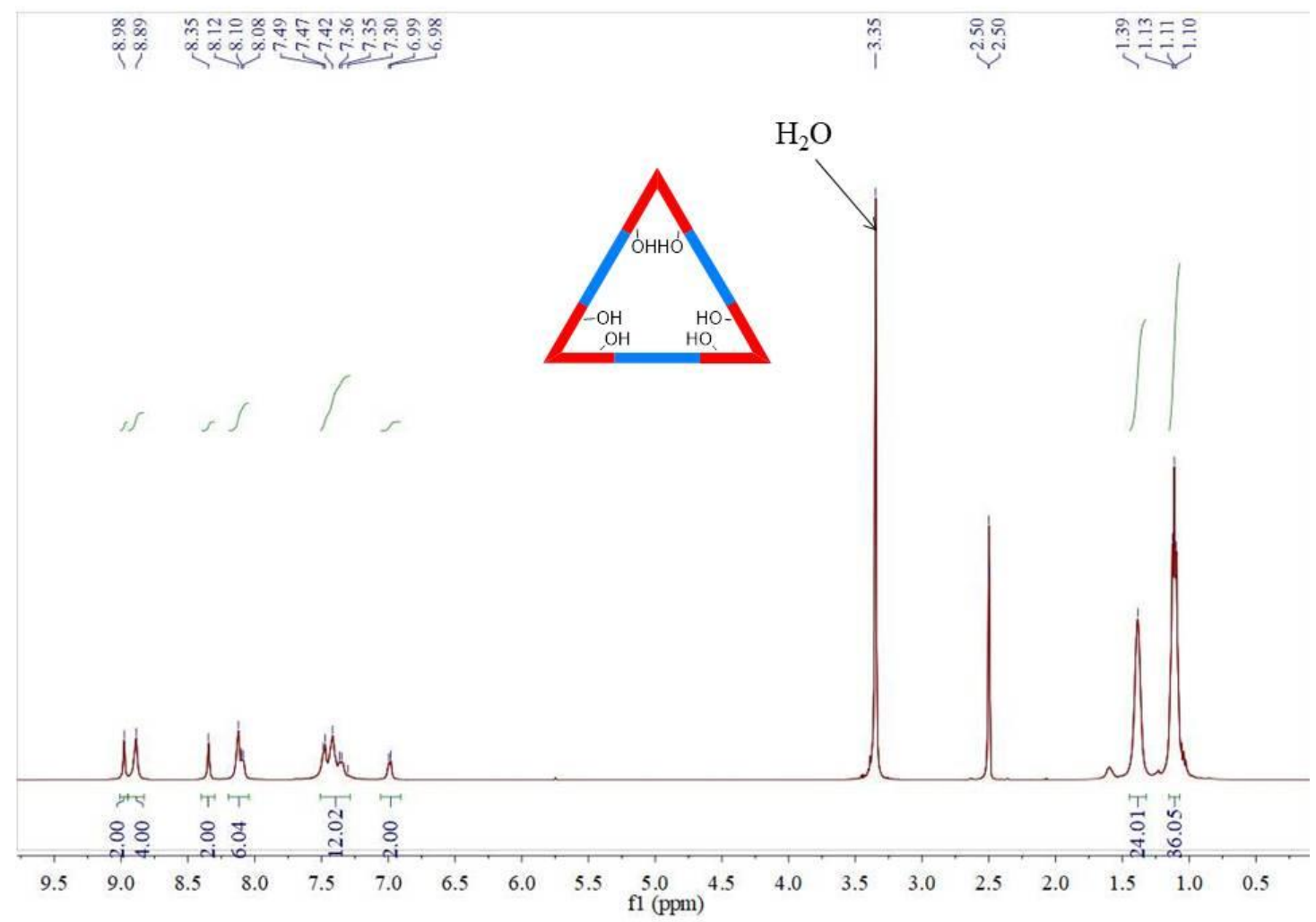

Figure S17. ${ }^{1} \mathrm{H}$ NMR $\left(500 \mathrm{MHz}, d_{6}\right.$-DMSO, $\left.22{ }^{\circ} \mathrm{C}\right)$ spectrum of $(S)-7 \mathbf{b}$.

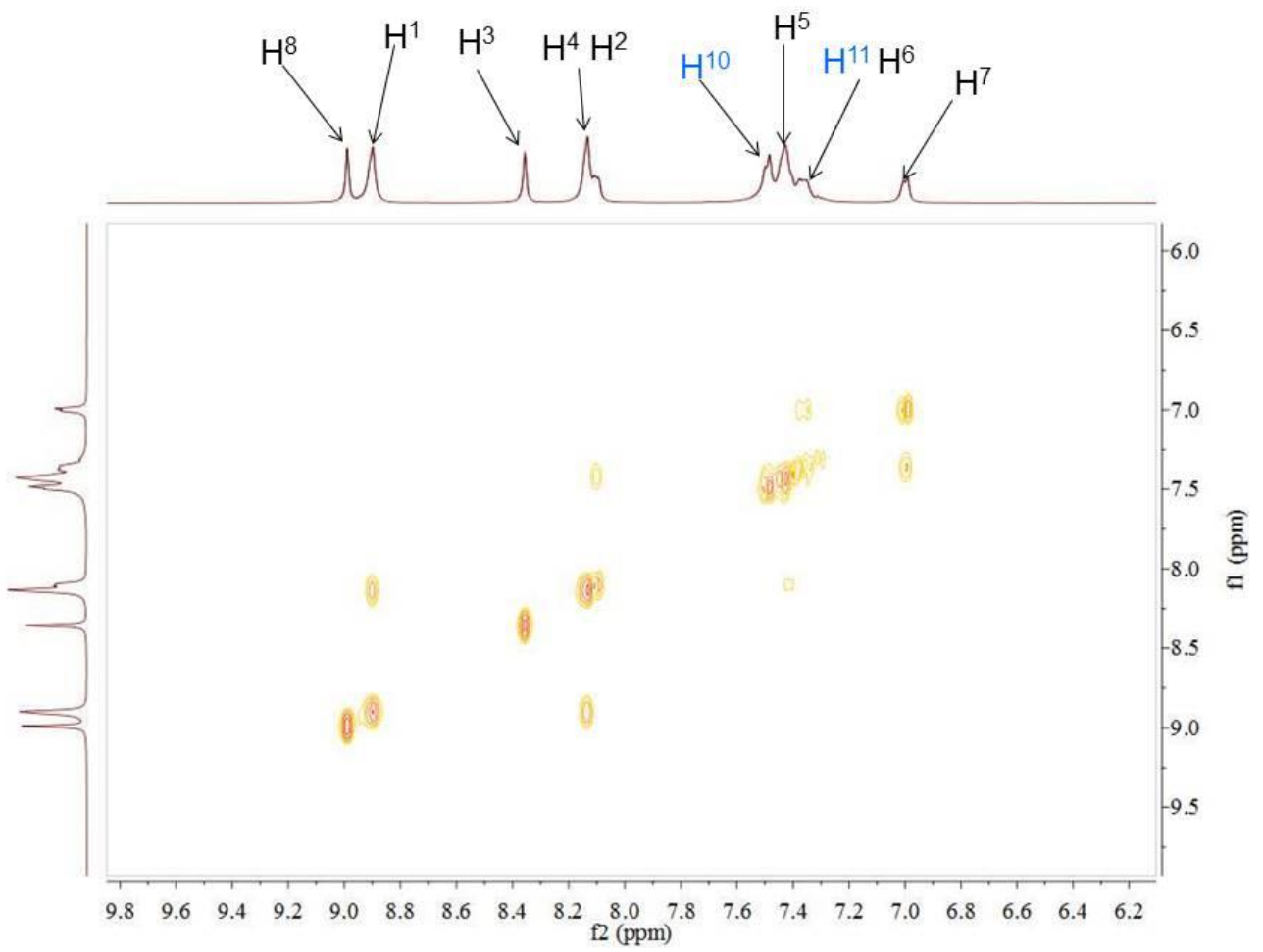

Figure S18. ${ }^{1} \mathrm{H}-{ }^{1} \mathrm{H}$ COSY $\left(500 \mathrm{MHz}, d_{6}\right.$-DMSO, $\left.22^{\circ} \mathrm{C}\right)$ spectrum of $(S)-7 \mathbf{b}$. 


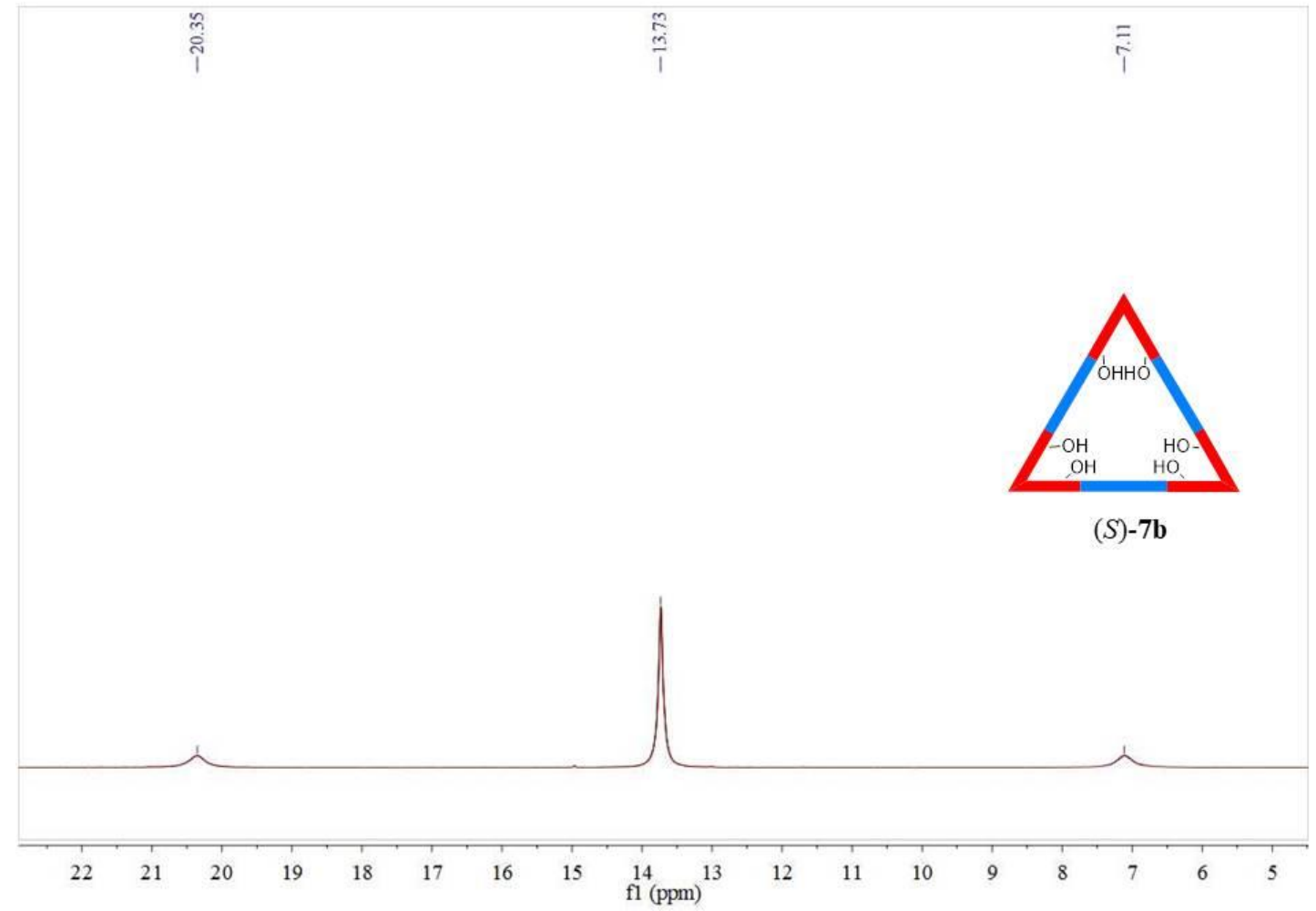

Figure S19. ${ }^{31} \mathrm{P}\left\{{ }^{1} \mathrm{H}\right\}$ NMR $\left(202 \mathrm{MHz}, d_{6}\right.$-DMSO, $\left.22{ }^{\circ} \mathrm{C}\right)$ spectrum of $(S)-7 \mathbf{b}$. 

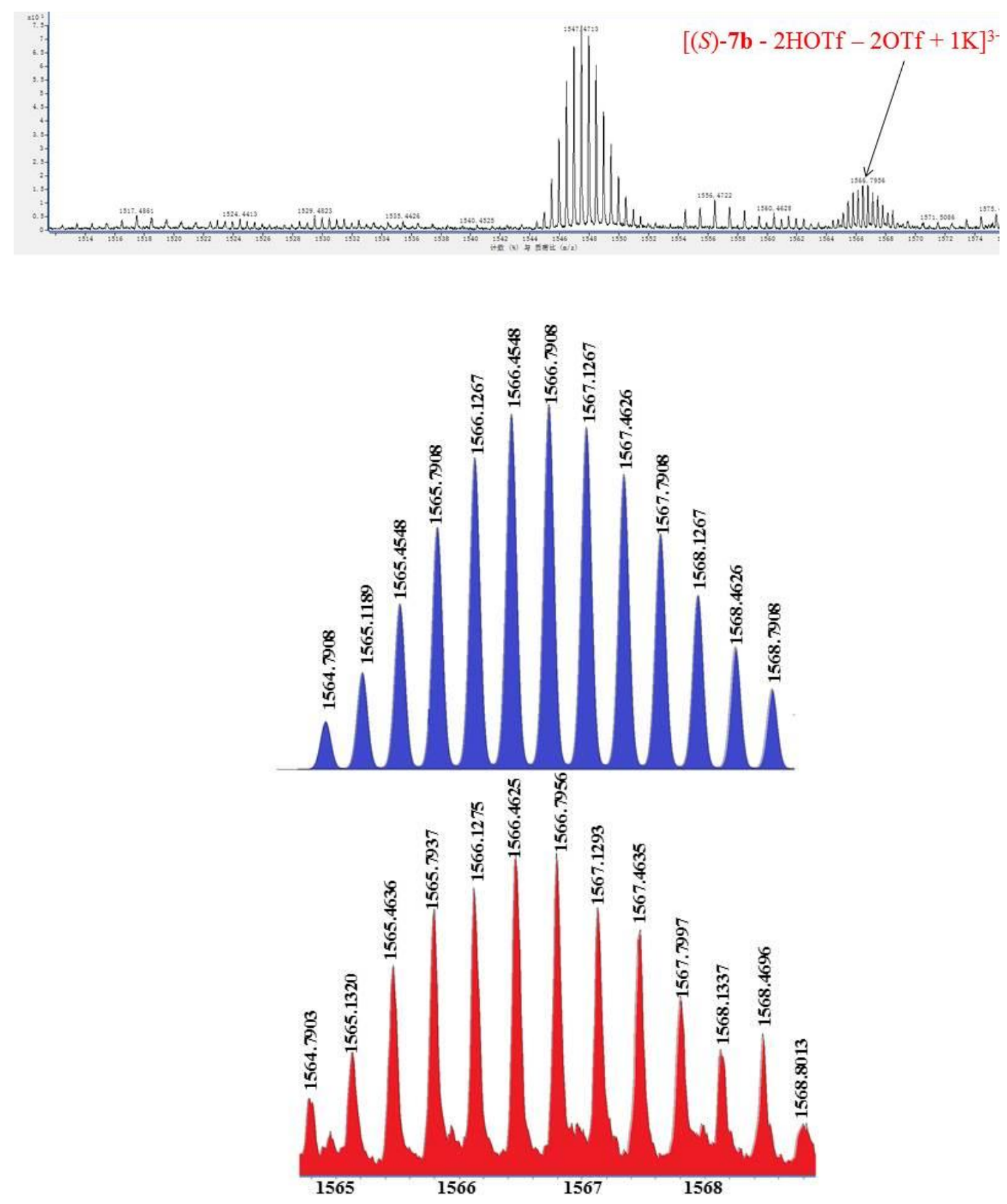

Figure S20. ESI-MS spectrum of $[(S)-7 \mathbf{b}-2 \mathrm{HOTf}-2 \mathrm{OTf}+1 \mathrm{~K}]^{3+}$ at $m / z=1566.4625(\mathrm{red})$ and its simulated spectrum (blue). 


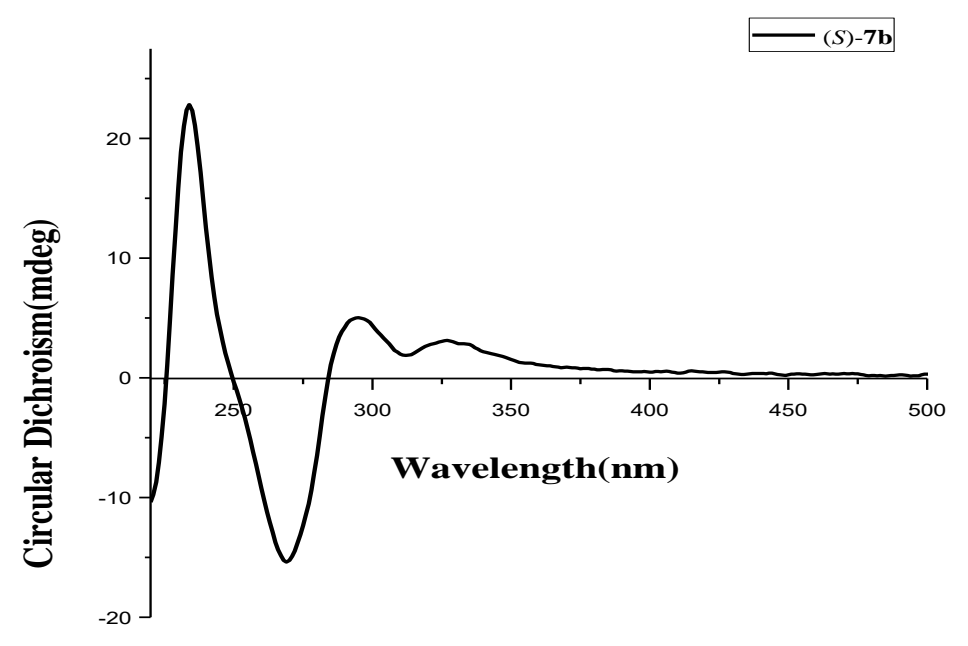

Figure S21. CD spectrum of $(S)$-7b in $\mathrm{CH}_{2} \mathrm{Cl}_{2}$.

8. General procedure for catalytic conjugate addition of $\alpha, \beta$-enones

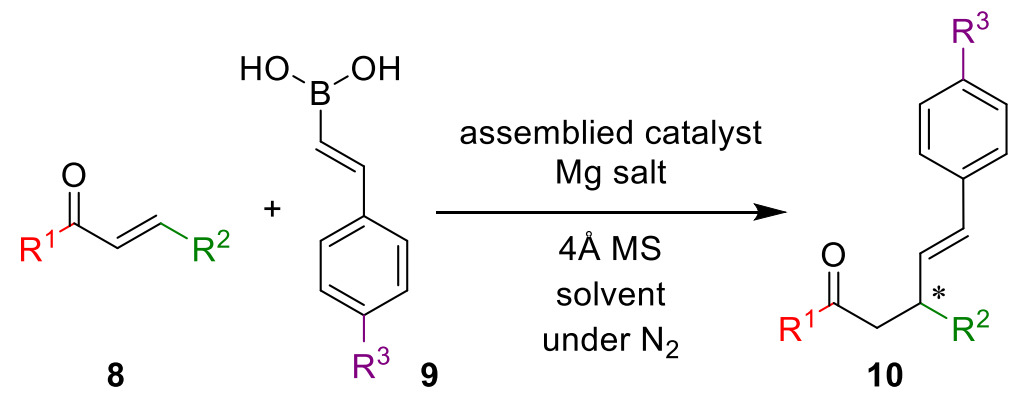

Under a nitrogen atmosphere, a $10 \mathrm{~mL}$ oven-dried Schlenk flask was charged with $\alpha, \beta$-enone $8(0.03 \mathrm{mmol})$, boronic acid $9(0.036 \mathrm{mmol}), \mathrm{Mg}$ salt, $4 \AA \mathrm{MS}$, the self-assemblied catalyst, and anhydrous solvent. The mixture was refluxed and monitored by TLC analysis. After the reaction mixture was cooled to room temperature, the solvent was evaporated. The residue was purified by silica gel column chromatography.

The conversions were determined by integration of the addition products as compared with the integration of the starting enones in ${ }^{1} \mathrm{H}$ NMR spectra of the reaction mixtures. The isolated yields were calculated after separation of the addition products. Enantioselectivities of the adducts were measured by chiral HPLC analysis using a chiral stationary phase column. 
<smiles>O=C(CC(C=Cc1ccccc1)c1ccccc1)c1ccccc1</smiles>

$(3 R, 4 E)-1,3,5$-triphenylpent-4-en-1-one (10a) ${ }^{\mathrm{S} 5, \mathrm{~S} 8}$

According to the general procedure, the reaction of chalcone (8a) $(6.95 \mathrm{mg})$ and (E)-styrylboronic acid (9a) $(5.98 \mathrm{mg})$ for $40 \mathrm{~h}$ gave the adduct $\mathbf{1 0 a}(9.38 \mathrm{mg}, 90 \%, 94 \%$ ee $(R))$. The spectral data were consistent with the literature. ${ }^{\mathrm{S}, \mathrm{S} 8} \mathrm{Mp} 81.3-83.3{ }^{\circ} \mathrm{C} .{ }^{1} \mathrm{H}$ NMR $\left(500 \mathrm{MHz}, \mathrm{CDCl}_{3}, 22{ }^{\circ} \mathrm{C}\right) \delta 7.94(\mathrm{~d}, J=7.3 \mathrm{~Hz}, 2 \mathrm{H}), 7.55(\mathrm{t}, J=7.4 \mathrm{~Hz}, 1 \mathrm{H}), 7.45(\mathrm{t}, J=7.7$ $\mathrm{Hz}, 2 \mathrm{H}), 7.32-7.29(\mathrm{~m}, 7 \mathrm{H}), 7.25-7.15(\mathrm{~m}, 3 \mathrm{H}), 6.44-6.37$ (m, 2H), 4.33-4.29 (m, 1H), 3.55$3.45(\mathrm{~m}, 2 \mathrm{H}) .{ }^{13} \mathrm{C} \mathrm{NMR}\left(125 \mathrm{MHz}, \mathrm{CDCl}_{3}, 22{ }^{\circ} \mathrm{C}\right) \delta 198.15,143.30,137.19,137.15,133.07$, $132.59,130.08,128.68,128.61,128.45,128.09,127.77,127.24,126.64,126.24,44.51,43.94$. ESI-TOF-MS $(\mathrm{m} / \mathrm{z})$ Calcd. for $\mathrm{C}_{23} \mathrm{H}_{21} \mathrm{O}[\mathrm{M}+\mathrm{H}]^{+}:$313.1587; Found: 313.1580. $[\alpha]_{\mathrm{D}}^{20}=+8(c=$ 0.05 in $\mathrm{CHCl}_{3}$ ) for a sample with $94 \%$ ee. The absolute configuration was determined by HPLC (Chiralpak IC, hexane/2-propanol $=98.5 / 1.5$, flow rate $=1.0 \mathrm{~mL} / \mathrm{min}$, UV detection at $\lambda=254 \mathrm{~nm}) ; t_{\mathrm{R}}=7.671 \min (S$, minor $), 8.335 \min (R$, major $)$.<smiles>CCC[C@H](/C=C/c1ccccc1)CC(C)=O</smiles>

$(R)$-4-styrylheptan-2-one (10b) $)^{\mathrm{S} 6}$

According to the general procedure, the reaction of $(E)$-hept-3-en-2-one $(\mathbf{8 b})(3.74 \mathrm{mg})$ and (E)-styrylboronic acid (9a) $(5.98 \mathrm{mg})$ for $72 \mathrm{~h}$ gave the adduct $\mathbf{1 0 b}(4.26 \mathrm{mg}, 60 \%, 87 \%$ ee $(R))$. The spectral data were consistent with the literature. ${ }^{\mathrm{S} 6}{ }^{1} \mathrm{H} \mathrm{NMR}\left(500 \mathrm{MHz}, \mathrm{CDCl}_{3}\right.$, $\left.22{ }^{\circ} \mathrm{C}\right) \delta 7.34-7.27(\mathrm{~m}, 4 \mathrm{H}), 7.20(\mathrm{t}, J=7.2 \mathrm{~Hz}, 1 \mathrm{H}), 6.38(\mathrm{~d}, J=15.9 \mathrm{~Hz}, 1 \mathrm{H}), 6.02-5.97(\mathrm{~m}$, 1H), 2.76-2.72 (m, 1H), $2.52(\mathrm{~d}, J=6.9 \mathrm{~Hz}, 2 \mathrm{H}), 2.13(\mathrm{~s}, 3 \mathrm{H}), 1.37-1.25(\mathrm{~m}, 4 \mathrm{H}), 0.90(\mathrm{t}, J=$ $7.0 \mathrm{~Hz}, 3 \mathrm{H}) .{ }^{13} \mathrm{C} \mathrm{NMR}\left(125 \mathrm{MHz}, \mathrm{CDCl}_{3,} 22{ }^{\circ} \mathrm{C}\right) \delta 208.11,137.35,133.19,130.22,128.49$, 127.12, 126.09, 49.52, 38.76, 37.30, 30.70, 20.35, 14.02. ESI-TOF-MS $(\mathrm{m} / z)$ Calcd. for $\mathrm{C}_{15} \mathrm{H}_{21} \mathrm{O}[\mathrm{M}+\mathrm{H}]^{+}:$217.1587; Found: 217.1594. $[\alpha]_{\mathrm{D}}^{20}=-8.44\left(c=0.05\right.$ in $\left.\mathrm{CHCl}_{3}\right)$ for a sample with $87 \%$ ee. The absolute configuration was determined by HPLC (Chiralpak AD-H, 
hexane $/ 2$-propanol $=99.5 / 0.5,1.0 \mathrm{~mL} / \mathrm{min}, \lambda=245 \mathrm{~nm}) ; t_{\mathrm{R}}=8.238 \mathrm{~min}(S$, minor $), 9.176 \mathrm{~min}$ $(R$, major $)$.<smiles>CCCCCC(C=Cc1ccccc1)CC(C)=O</smiles>

(E)-4-styrylnonan-2-one (10c)

According to the general procedure, the reaction of $(E)$-non-3-en-2-one $(\mathbf{8 c})(3.74 \mathrm{mg})$ and (E)-styrylboronic acid (9a) (5.98 mg) at for $72 \mathrm{~h}$ gave the adduct 10c $(3.26 \mathrm{mg}, 40 \%, 87 \% \mathrm{ee})$. ${ }^{1} \mathrm{H}$ NMR $\left(500 \mathrm{MHz}, \mathrm{CDCl}_{3}, 22{ }^{\circ} \mathrm{C}\right) \delta 7.34-7.27(\mathrm{~m}, 4 \mathrm{H}), 7.20(\mathrm{t}, J=7.2 \mathrm{~Hz}, 1 \mathrm{H}), 6.38(\mathrm{~d}, J=$ $15.8 \mathrm{~Hz}, 1 \mathrm{H}), 6.02-5.97(\mathrm{~m}, 1 \mathrm{H}), 2.74-2.70(\mathrm{~m}, 1 \mathrm{H}), 2.52(\mathrm{~d}, J=6.9 \mathrm{~Hz}, 2 \mathrm{H}), 2.13(\mathrm{~s}, 3 \mathrm{H})$, $1.33-1.27(\mathrm{~m}, 8 \mathrm{H}), 0.87(\mathrm{t}, J=6.7 \mathrm{~Hz}, 3 \mathrm{H}) .{ }^{13} \mathrm{C} \mathrm{NMR}\left(125 \mathrm{MHz}, \mathrm{CDCl}_{3}, 22{ }^{\circ} \mathrm{C}\right) \delta 208.13$, $137.39,133.28,130.21,128.50,127.12,126.11,49.55,39.01,35.10,31.82,30.70,26.89$, 22.59, 14.08. ESI-TOF-MS $(\mathrm{m} / \mathrm{z})$ Calcd. For $\mathrm{C}_{17} \mathrm{H}_{25} \mathrm{O}[\mathrm{M}+\mathrm{H}]^{+}:$: 245.1900; Found: 245.1898. $[\alpha]_{\mathrm{D}}^{20}=-15.33\left(c=0.05\right.$ in $\left.\mathrm{CHCl}_{3}\right)$ for a sample with $87 \%$ ee. The absolute configuration was determined by HPLC (Chiralpak AD-H, hexane/2-propanol = 99.5/0.5, $1.0 \mathrm{~mL} / \mathrm{min}, \lambda=245$ $\mathrm{nm}) ; t_{\mathrm{R}}=7.816 \min$ (minor), $8.292 \min$ (major).<smiles>CC(=O)C[C@H](C=Cc1ccccc1)c1ccccc1</smiles>

(4R,5E)-4,6-diphenylhex-5-en-2-one (10d) $)^{\mathrm{S} 7, \mathrm{~S} 8}$

According to the general procedure, the reaction of $(E)$-4-phenylbut-3-en-2-one $(\mathbf{8 d})(4.88$ $\mathrm{mg}$ ) and (E)-styrylboronic acid (9a) $(5.98 \mathrm{mg})$ for $40 \mathrm{~h}$ gave the adduct $10 \mathrm{~d}(5.43 \mathrm{mg}, 65 \%$, 94\% ee $(R))$. The spectral data were consistent with the literature. ${ }^{\mathrm{S}, \mathrm{S} 8}{ }^{1} \mathrm{H}$ NMR $(500 \mathrm{MHz}$, $\left.\mathrm{CDCl}_{3}, 22{ }^{\circ} \mathrm{C}\right){ }^{1} \mathrm{H}$ NMR $\left(500 \mathrm{MHz}, \mathrm{CDCl}_{3}\right) \delta$ 7.26-7.20 (m, 7H), 7.17-7.11 (m, 3H), 6.33$6.23(\mathrm{~m}, 2 \mathrm{H}), 4.03-3.99(\mathrm{~m}, 1 \mathrm{H}), 2.89-2.85(\mathrm{~m}, 2 \mathrm{H}), 2.04(\mathrm{~s}, 3 \mathrm{H}) .{ }^{13} \mathrm{C}$ NMR (125 MHz, $\left.\mathrm{CDCl}_{3}\right) \delta 206.90,142.97,137.11,132.38,130.02,128.72,128.50,127.66,127.34,126.71$, 126.26, 49.44, 43.98, 30.76. ESI-TOF-MS $(\mathrm{m} / \mathrm{z})$ Calcd. For $\mathrm{C}_{18} \mathrm{H}_{19} \mathrm{O}[\mathrm{M}+\mathrm{H}]^{+}$: 251.1430; Found: $251.1433 .[\alpha]_{\mathrm{D}}^{20}=+15.33\left(c=0.05\right.$ in $\left.\mathrm{CHCl}_{3}\right)$ for a sample with $94 \%$ ee. The absolute 
configuration was determined by HPLC (Chiralpak AD-H, hexane/2-propanol = 98.5/1.5, 1.0 $\mathrm{mL} / \min , \lambda=245 \mathrm{~nm}) ; t_{\mathrm{R}}=12.881 \min (R$, major $), 13.830 \min (S$, minor $)$.<smiles>COc1ccc(C(C=Cc2ccccc2)CC(C)=O)cc1</smiles>

(E)-4-(4-methoxyphenyl)-6-phenylhex-5-en-2-one (10e) ${ }^{\text {S9 }}$

According to the general procedure, the reaction of (E)-4-(4-methoxyphenyl)but-3-en-2-one (8e) $(5.52 \mathrm{mg})$ and $(E)$-styrylboronic acid $(\mathbf{9 a})(5.98 \mathrm{mg})$ for $40 \mathrm{~h}$ gave the adduct $10 \mathrm{e}(5.43$ $\mathrm{mg}, 65 \%, 96 \%$ ee). The spectral data were consistent with the literature. ${ }^{59}{ }^{1} \mathrm{H}$ NMR $(500 \mathrm{MHz}$, $\left.\mathrm{CDCl}_{3}, 22{ }^{\circ} \mathrm{C}\right) \delta 7.25-7.19(\mathrm{~m}, 4 \mathrm{H}), 7.12-7.09(\mathrm{~m}, 3 \mathrm{H}), 6.80-6.77(\mathrm{~m}, 2 \mathrm{H}), 6.30-6.21(\mathrm{~m}$, 2H), 3.98-3.94 (m, 1H), $3.72(\mathrm{~s}, 3 \mathrm{H}), 2.89-2.80(\mathrm{~m}, 2 \mathrm{H}), 2.03$ (s, 3H). ${ }^{13} \mathrm{C}$ NMR (125 MHz, $\left.\mathrm{CDCl}_{3}, 22{ }^{\circ} \mathrm{C}\right) \delta 207.13,158.31,137.15,134.92,132.71,129.68,128.62,128.49,127.28$, 126.22, 114.09, 55.27, 49.58, 43.17, 30.78. ESI-TOF-MS (m/z) Calcd. for $\mathrm{C}_{19} \mathrm{H}_{21} \mathrm{O}_{2}[\mathrm{M}+\mathrm{H}]^{+}$: 281.1536; Found: 281.1541. $[\alpha]_{\mathrm{D}}^{20}=+24.7\left(c=0.05\right.$ in $\left.\mathrm{CHCl}_{3}\right)$ for a sample with $96 \%$ ee. The absolute configuration was determined by HPLC (Chiralpak AD-H, hexane/2-propanol = 98.5/1.5, $1.0 \mathrm{~mL} / \mathrm{min}, \lambda=245 \mathrm{~nm}$ ); $t_{\mathrm{R}}=16.908 \mathrm{~min}$ (minor), 18.677 min (major).<smiles>CC(=O)CC(C=Cc1ccccc1)c1ccc(Cl)cc1</smiles>

(E)-4-(4-chlorophenyl)-6-phenylhex-5-en-2-one (10f)

According to the general procedure, the reaction of (E)-4-(4-chlorophenyl)but-3-en-2-one (8f) $(6.03 \mathrm{mg})$ and $(E)$-styrylboronic acid $(\mathbf{9 a})(5.98 \mathrm{mg})$ for $72 \mathrm{~h}$ gave the adduct $\mathbf{1 0 f}(5.52 \mathrm{mg}$, 59\%, 95\% ee ). ${ }^{1} \mathrm{H}$ NMR (500 MHz, $\left.\mathrm{CDCl}_{3}, 22{ }^{\circ} \mathrm{C}\right) \delta 7.32-7.26(\mathrm{~m}, 6 \mathrm{H}), 7.20-7.18(\mathrm{~m}, 3 \mathrm{H})$, 6.37-6.25 (m, 2H), 4.09-4.04 (m, 1H), 2.98-2.87 (m, 2H), 2.11 (s, 3H). ${ }^{13} \mathrm{C}$ NMR (125 MHz, $\left.\mathrm{CDCl}_{3}, 22{ }^{\circ} \mathrm{C}\right) \delta 206.47,141.44,136.87,132.41,131.80,130.33,129.07,128.81,128.54$, 127.49, 126.24, 49.22, 43.15, 30.75. ESI-TOF-MS $(\mathrm{m} / \mathrm{z})$ Calcd. for $\mathrm{C}_{18} \mathrm{H}_{17} \mathrm{ClNaO}[\mathrm{M}+\mathrm{Na}]^{+}$: 
307.0860; Found: 307.0861. $[\alpha]_{\mathrm{D}}^{20}=+16.32\left(c=0.05\right.$ in $\left.\mathrm{CHCl}_{3}\right)$ for a sample with $95 \%$ ee. The absolute configuration was determined by HPLC (Chiralpak AD-H, hexane/2-propanol = 99.4/0.6, $1.0 \mathrm{~mL} / \mathrm{min}, \lambda=245 \mathrm{~nm}$ ); $t_{\mathrm{R}}=20.382 \mathrm{~min}$ (minor), $21.570 \mathrm{~min}$ (major).<smiles>CC(=O)C[C](C=Cc1ccccc1)c1ccc(Cl)cc1Cl</smiles>

(E)-4-(2,4-dichlorophenyl)-6-phenylhex-5-en-2-one (10g)

According to the general procedure, the reaction of $(E)$-4-(2,4-dichlorophenyl)but-3-en-2-one $(\mathbf{8 g})(6.03 \mathrm{mg})$ and $(E)$-styrylboronic acid $(\mathbf{9 a})(5.98 \mathrm{mg})$ for $72 \mathrm{~h}$ gave the adduct $\mathbf{1 0 g}(5.70$ $\mathrm{mg}, 60 \%, 95 \%$ ee $).{ }^{1} \mathrm{H}$ NMR $\left(500 \mathrm{MHz}, \mathrm{CDCl}_{3}, 22{ }^{\circ} \mathrm{C}\right) \delta 7.40(\mathrm{~d}, J=1.6 \mathrm{~Hz}, 1 \mathrm{H}), 7.33-7.27$ (m, 4H), 7.23-7.20 (m, 3H), 6.39-6.24 (m, 2H), 4.57-4.52 (m, 1H), 3.00-2.89 (m, 2H), 2.16 (s, 3H). ${ }^{13} \mathrm{C}$ NMR $\left(125 \mathrm{MHz}, \mathrm{CDCl}_{3}, 22{ }^{\circ} \mathrm{C}\right) \delta 205.94,138.99,136.76,134.39,132.90,131.19$, 129.86, 129.77, 129.44, 128.55, 127.60, 127.40, 126.30, 48.25, 39.70, 30.22. ESI-TOF-MS $(m / z)$ Calcd. for $\mathrm{C}_{18} \mathrm{H}_{17} \mathrm{Cl}_{2} \mathrm{O}[\mathrm{M}+\mathrm{H}]^{+}: 319.0651$; Found: $319.0629 .[\alpha]_{\mathrm{D}}^{20}=-5.33(c=0.05$ in $\mathrm{CHCl}_{3}$ ) for a sample with $95 \%$ ee. The absolute configuration was determined by HPLC $($ Chiralpak OD-H, hexane/2-propanol $=98.5 / 1.5,1.0 \mathrm{~mL} / \mathrm{min}, \lambda=245 \mathrm{~nm}) ; t_{\mathrm{R}}=12.943 \mathrm{~min}$ (minor), $17.198 \min$ (major).<smiles>COc1cc(C(C=Cc2ccccc2)CC(C)=O)ccc1O</smiles>

(E)-4-(4-hydroxy-3-methoxyphenyl)-6-phenylhex-5-en-2-one (10h)

According to the general procedure, the reaction of (E)-4-(2,4-dichlorophenyl)but-3-en-2-one (8h) $(6.03 \mathrm{mg})$ and $(E)$-styrylboronic acid $(\mathbf{9 a})(5.98 \mathrm{mg})$ for $40 \mathrm{~h}$ gave the adduct $\mathbf{1 0 h}(6.13$ $\mathrm{mg}, 62 \%, 94 \%$ ee $).{ }^{1} \mathrm{H}$ NMR $\left(500 \mathrm{MHz}, \mathrm{CDCl}_{3}, 22{ }^{\circ} \mathrm{C}\right) \delta 7.26-7.19(\mathrm{~m}, 4 \mathrm{H}), 7.13(\mathrm{t}, J=7.2$ $\mathrm{Hz}, 1 \mathrm{H}), 6.81-6.79(\mathrm{~m}, 1 \mathrm{H}), 6.69-6.68(\mathrm{~m}, 2 \mathrm{H}), 6.30-6.21$ (m, 2H), 5.44 (br, 1H), 3.96-3.91 $(\mathrm{m}, 1 \mathrm{H}), 3.81(\mathrm{~s}, 3 \mathrm{H}), 2.85-2.83(\mathrm{~m}, 2 \mathrm{H}), 2.04(\mathrm{~s}, 3 \mathrm{H}) .{ }^{13} \mathrm{C} \mathrm{NMR}\left(125 \mathrm{MHz}, \mathrm{CDCl}_{3,}, 22{ }^{\circ} \mathrm{C}\right) \delta$ 
207.16, 146.55, 144.33, 137.13, 134.86, 132.63, 129.70, 128.51, 127.32, 126.24, 119.97, $114.49,110.52,55.95,49.62,43.68,30.82$. ESI-TOF-MS $(\mathrm{m} / \mathrm{z})$ Calcd. for $\mathrm{C}_{19} \mathrm{H}_{20} \mathrm{NaO}[\mathrm{M}+$ $\mathrm{Na}]^{+}:$319.1305; Found: 319.1310. $[\alpha]_{\mathrm{D}}^{20}=+7.56\left(c=0.05\right.$ in $\left.\mathrm{CHCl}_{3}\right)$ for a sample with $94 \%$ ee. The absolute configuration was determined by HPLC (Chiralpak AD-H, hexane/2-propanol = 98.5/1.5, $1.0 \mathrm{~mL} / \mathrm{min}, \lambda=245 \mathrm{~nm}$ ); $t_{\mathrm{R}}=84.089 \mathrm{~min}$ (major), $96.538 \mathrm{~min}$ (minor).<smiles>COc1ccc(C(=O)C[C@H](/C=C/c2ccccc2)c2ccccc2)cc1</smiles>

(3R,4E)-1-(4-methoxyphenyl)-3,5-diphenylpent-4-en-1-one (10i) $)^{\mathrm{S} 8 \mathrm{~S} 10}$

According to the general procedure, the reaction of (E)-1-(4-methoxyphenyl)-3-phenylprop-2-en-1-one (8i) $(7.95 \mathrm{mg})$ and (E)-styrylboronic acid (9) $(5.98 \mathrm{mg})$ for $40 \mathrm{~h}$ gave the adduct $10 \mathrm{i}(9.71 \mathrm{mg}, 85 \%, 90 \%$ ee $(R))$. The spectral data were consistent with the literature. ${ }^{\mathrm{S} 8 \mathrm{~S} 10} \mathrm{Mp} 107.7-109.7{ }^{\circ} \mathrm{C} .{ }^{1} \mathrm{H}$ NMR $\left(500 \mathrm{MHz}, \mathrm{CDCl}_{3}\right.$, $\left.22{ }^{\circ} \mathrm{C}\right) \delta 7.84(\mathrm{~d}, J=8.9 \mathrm{~Hz}, 2 \mathrm{H}), 7.23-7.16(\mathrm{~m}, 7 \mathrm{H}), 7.16-7.06(\mathrm{~m}, 3 \mathrm{H}), 6.82(\mathrm{~d}, J=8.9 \mathrm{~Hz}$, 2H), 6.35-6.26 (m, 2H), 4.22-4.18 (m, 1H), 3.77 (s, 3H), 3.40-3.30 (m, 2H). ${ }^{13} \mathrm{C}$ NMR $(125$ $\left.\mathrm{MHz}, \mathrm{CDCl}_{3}, 22{ }^{\circ} \mathrm{C}\right) \delta 196.69,163.48,143.46,137.27,132.77,130.40,130.27,129.99$, 128.66, 128.44, 127.79, 127.20, 126.59, 126.25, 113.75, 55.48, 44.15, 44.09. ESI-TOF-MS $(m / z)$ Calcd. for $\mathrm{C}_{24} \mathrm{H}_{23} \mathrm{O}_{2}[\mathrm{M}+\mathrm{H}]^{+}:$343.1693; Found: 343.1697. $[\alpha]_{\mathrm{D}}^{20}=+7.47(c=0.05$ in $\mathrm{CHCl}_{3}$ ) for a sample with $90 \%$ ee. The absolute configuration was determined by HPLC $($ Chiralpak IC, hexane/2-propanol $=98.5 / 1.5,1.0 \mathrm{~mL} / \mathrm{min}, \lambda=245 \mathrm{~nm}) ; t_{\mathrm{R}}=6.973 \mathrm{~min}(S$, minor), $7.397 \min (R$, major).<smiles>O=C(C[C](C=Cc1ccccc1)c1ccccc1)c1ccc(O)cc1</smiles>

(E)-1-(4-hydroxyphenyl)-3,5-diphenylpent-4-en-1-one (10j)

According to the general procedure, the reaction of 
(E)-1-(4-hydroxyphenyl)-3-phenylprop-2-en-1-one $(\mathbf{8 j})(7.48 \mathrm{mg})$ and $(E)$-styrylboronic acid (9a) $\left(5.98 \mathrm{mg}\right.$ ) for $40 \mathrm{~h}$ gave the adduct $\mathbf{1 0 j}$ (7.89 mg, 72\%, 91\% ee). ${ }^{1} \mathrm{H}$ NMR (500 MHz, $\left.\mathrm{CDCl}_{3}, 22{ }^{\circ} \mathrm{C}\right) \delta 7.81(\mathrm{~d}, J=8.7 \mathrm{~Hz}, 2 \mathrm{H}), 7.24-7.22(\mathrm{~m}, 6 \mathrm{H}), 7.19-7.08(\mathrm{~m}, 4 \mathrm{H}), 6.78(\mathrm{~d}, J=$ $8.7 \mathrm{~Hz}, 2 \mathrm{H}), 6.36-6.28(\mathrm{~m}, 2 \mathrm{H}), 5.76(\mathrm{br}, 1 \mathrm{H}), 4.23-4.19(\mathrm{~m}, 1 \mathrm{H}), 3.41-3.32(\mathrm{~m}, 2 \mathrm{H}) .{ }^{13} \mathrm{C}$ NMR (125 MHz, $\left.\mathrm{CDCl}_{3,22}{ }^{\circ} \mathrm{C}\right) \delta 197.06,160.11,143.35,137.21,132.65,130.77,130.32$, 130.04, 128.68, 128.46, 127.77, 127.24, 126.63, 126.25, 115.39, 44.16. ESI-TOF-MS $(\mathrm{m} / z)$ Calcd. for $\mathrm{C}_{23} \mathrm{H}_{20} \mathrm{NaO}[\mathrm{M}+\mathrm{Na}]^{+}:$351.1356; Found: $351.1355 .[\alpha]_{\mathrm{D}}^{20}=+4.67(c=0.05$ in $\mathrm{CHCl}_{3}$ ) for a sample with $91 \%$ ee. The absolute configuration was determined by HPLC $($ Chiralpak OD-H, hexane/2-propanol $=90 / 10,1.0 \mathrm{~mL} / \mathrm{min}, \lambda=245 \mathrm{~nm}) ; t_{\mathrm{R}}=8.511 \mathrm{~min}$ (minor), 17.174 min (major).<smiles>O=C(C[C](C=Cc1ccccc1)c1ccccc1)c1ccc(F)cc1</smiles>

(E)-1-(4-fluorophenyl)-3,5-diphenylpent-4-en-1-one (10k) $)^{\mathrm{S} 9}$

According to the general procedure, the reaction of (E)-1-(4-fluorophenyl)-3-phenylprop-2-en-1-one $(\mathbf{8 k})(7.55 \mathrm{mg})$ and $(E)$-styrylboronic acid (9a) $(5.98 \mathrm{mg})$ for $40 \mathrm{~h}$ gave the adduct 10k $(9.26 \mathrm{mg}, 84 \%, 95 \% \mathrm{ee})$. The spectral data were consistent with the literature. ${ }^{\mathrm{S} 9}{ }^{1} \mathrm{H}$ NMR $\left(500 \mathrm{MHz}, \mathrm{CDCl}_{3}, 22{ }^{\circ} \mathrm{C}\right) \delta 7.91-7.88(\mathrm{~m}, 2 \mathrm{H})$, 7.25-7.19 (m, 7H), 7.17-7.10 (m, 3H), 7.06-7.02 (m, 2H), 6.36-6.29 (m, 2H), 4.23-4.19 (m, 1H), 3.44-3.35 (m, 2H). ${ }^{13} \mathrm{C}$ NMR $\left(125 \mathrm{MHz}, \mathrm{CDCl}_{3}, 22{ }^{\circ} \mathrm{C}\right) \delta 196.59,165.75\left(\mathrm{~d}, J_{\mathrm{F}-\mathrm{C}}=254\right.$ $\mathrm{Hz}), 143.17,137.13,133.58\left(\mathrm{~d}, J_{\mathrm{F}-\mathrm{C}}=1.3 \mathrm{~Hz}\right), 132.43,130.74\left(\mathrm{~d}, J_{\mathrm{F}-\mathrm{C}}=8.8 \mathrm{~Hz}\right), 130.17$, $128.72,128.48,127.75,127.31,126.71,126.25,115.72\left(\mathrm{~d}, J_{\mathrm{F}-\mathrm{C}}=22.5 \mathrm{~Hz}\right), 44.44,44.00$. ESI-TOF-MS $(\mathrm{m} / \mathrm{z})$ Calcd. for $\mathrm{C}_{23} \mathrm{H}_{20} \mathrm{FO}[\mathrm{M}+\mathrm{H}]^{+}: 331.1493$; Found: $331.1500 .[\alpha]_{\mathrm{D}}^{20}=+6.42$ ( $c=0.05$ in $\mathrm{CHCl}_{3}$ ) for a sample with $95 \%$ ee. The absolute configuration was determined by HPLC (Chiralpak IC, hexane/2-propanol $=98.5 / 1.5,1.0 \mathrm{~mL} / \mathrm{min}, \lambda=245 \mathrm{~nm}) ; t_{\mathrm{R}}=17.757$ $\min$ (minor), $19.226 \min$ (major). 
<smiles>COc1ccc(C(C=Cc2ccccc2)CC(=O)c2ccccc2)cc1</smiles>

(3R,4E)-3-(4-methoxyphenyl)-1,5-diphenylpent-4-en-1-one (10I) ${ }^{\mathrm{S} 8, \mathrm{~S} 11}$

According to the general procedure, the reaction of (E)-3-(4-methoxyphenyl)-1-phenylprop-2-en-1-one (81) $(7.95 \mathrm{mg})$ and (E)-styrylboronic acid (9a) $(5.98 \mathrm{mg})$ for $40 \mathrm{~h}$ gave the adduct $101(9.26 \mathrm{mg}, 98 \%, 95 \%$ ee $(R))$. The spectral data were consistent with the literature. ${ }^{\mathrm{S} 8 \mathrm{~S} 11} \mathrm{Mp} 85.0-87.1^{\circ} \mathrm{C} .{ }^{1} \mathrm{H} \mathrm{NMR}\left(500 \mathrm{MHz}, \mathrm{CDCl}_{3}, 22{ }^{\circ} \mathrm{C}\right)$ $\delta 7.84(\mathrm{~d}, J=7.4 \mathrm{~Hz}, 2 \mathrm{H}), 7.45(\mathrm{t}, J=7.4 \mathrm{~Hz}, 1 \mathrm{H}), 7.35(\mathrm{t}, J=7.7 \mathrm{~Hz}, 2 \mathrm{H}), 7.21-7.17(\mathrm{~m}$, 3H), 7.14-7.07 (m, 4H), $6.76(\mathrm{~d}, J=8.6 \mathrm{~Hz}, 2 \mathrm{H}), 6.33-6.25(\mathrm{~m}, 2 \mathrm{H}), 4.18-4.14(\mathrm{~m}, 1 \mathrm{H}), 3.69$ (s, 3H), 3.42-3.33 (m, 2H). ${ }^{13} \mathrm{C}$ NMR (125 MHz, $\left.\mathrm{CDCl}_{3,} 22{ }^{\circ} \mathrm{C}\right) \delta 198.33,158.27,137.27$, $137.20,135.30,133.06,132.95,129.77,128.74,128.62,128.45,128.11,127.20,126.24$, 114.07, 55.28, 44.66, 43.15. ESI-TOF-MS $(\mathrm{m} / \mathrm{z})$ Calcd. for $\mathrm{C}_{24} \mathrm{H}_{23} \mathrm{O}_{2}[\mathrm{M}+\mathrm{H}]^{+}:$343.1693; Found: $343.1691 .[\alpha]_{\mathrm{D}}^{20}=+11.35\left(c=0.05\right.$ in $\left.\mathrm{CHCl}_{3}\right)$ for a sample with $95 \%$ ee. The absolute configuration was determined by HPLC (Chiralpak IC, hexane/2-propanol $=98.5 / 1.5,1.0$ $\mathrm{mL} / \mathrm{min}, \lambda=245 \mathrm{~nm}) ; t_{\mathrm{R}}=12.908 \min (S$, minor $), 13.860 \min (R$, major $)$.<smiles>O=C(CC(=Cc1ccccc1)c1ccc(O)cc1)c1ccccc1</smiles>

(E)-3-(4-hydroxyphenyl)-1,5-diphenylpent-4-en-1-one (10m)

According to the general procedure, the reaction of (E)-3-(4-hydroxyphenyl)-1-phenylprop-2-en-1-one (8m) (7.48 mg) and (E)-styrylboronic acid (9a) $\left(5.98 \mathrm{mg}\right.$ ) for $40 \mathrm{~h}$ gave the adduct 10m (9.97mg, 91\%, 96\% ee). ${ }^{1} \mathrm{H}$ NMR (500 MHz, $\left.\mathrm{CDCl}_{3}, 22{ }^{\circ} \mathrm{C}\right) \delta 7.94(\mathrm{~d}, J=7.2 \mathrm{~Hz}, 2 \mathrm{H}), 7.55(\mathrm{t}, J=7.4 \mathrm{~Hz}, 1 \mathrm{H}), 7.45(\mathrm{t}, J=7.7 \mathrm{~Hz}, 2 \mathrm{H})$, 7.31-7.24 (m, 4H), 7.19-7.17(m, 3H), 6.78 (d, $J=8.6 \mathrm{~Hz}, 2 \mathrm{H}), 6.42-6.34(\mathrm{~m}, 2 \mathrm{H}), 4.72(\mathrm{br}$, $1 \mathrm{H}), 4.26-4.22(\mathrm{~m}, 1 \mathrm{H}), 3.51-3.42(\mathrm{~m}, 2 \mathrm{H}) .{ }^{13} \mathrm{C} \mathrm{NMR}\left(125 \mathrm{MHz}, \mathrm{CDCl}_{3,} 22{ }^{\circ} \mathrm{C}\right) \delta 198.45$, 
$154.18,137.22,137.15,135.41,133.10,132.88,129.78,128.95,128.62,128.46,128.11$, 127.22, $126.23,115.48,44.65,43.17$. ESI-TOF-MS $(\mathrm{m} / \mathrm{z})$ Calcd. for $\mathrm{C}_{23} \mathrm{H}_{20} \mathrm{NaO}[\mathrm{M}+\mathrm{Na}]^{+}$: 351.1356; Found: $351.1351 .[\alpha]_{\mathrm{D}}^{20}=+8.67\left(c=0.05\right.$ in $\left.\mathrm{CHCl}_{3}\right)$ for a sample with $96 \%$ ee. The absolute configuration was determined by HPLC (Chiralpak OD-H, hexane/2-propanol = 90/10, $1.0 \mathrm{~mL} / \mathrm{min}, \lambda=245 \mathrm{~nm}$ ); $t_{\mathrm{R}}=20.854 \mathrm{~min}$ (minor), $28.267 \mathrm{~min}$ (major).<smiles>O=C(C[C@H](C=Cc1ccccc1)c1ccc(Cl)cc1)c1ccccc1</smiles>

(3R,4E)-3-(4-chlorophenyl)-1,5-diphenylpent-4-en-1-one (10n) ${ }^{\mathrm{S} 8, \mathrm{~S} 12}$

According to the general procedure, the reaction of (E)-3-(4-chlorophenyl)-1-phenylprop-2-en-1-one (8n) $(8.10 \mathrm{mg})$ and (E)-styrylboronic acid (9a) $(5.98 \mathrm{mg})$ for $40 \mathrm{~h}$ gave the adduct $10 \mathrm{n}(5.79 \mathrm{mg}, 50 \%, 94 \%$ ee $(R))$. The spectral data were consistent with the literature. ${ }^{\mathrm{s}, \mathrm{S} 12}{ }^{1} \mathrm{H}$ NMR $\left(500 \mathrm{MHz}, \mathrm{CDCl}_{3}, 22{ }^{\circ} \mathrm{C}\right): \delta 7.93(\mathrm{~d}, J=7.2$ Hz, 2H), 7.56 (t, $J=7.4 \mathrm{~Hz}, 1 \mathrm{H}), 7.45(\mathrm{t}, J=7.7 \mathrm{~Hz}, 2 \mathrm{H}), 7.31-7.26(\mathrm{~m}, 7 \mathrm{H}), 7.24-7.18(\mathrm{~m}$, 2H), 6.40-6.34 (m, 2H), 4.34-4.24 (m, 1H), 3.53-3.43 (m, 2H). ${ }^{13} \mathrm{C} \mathrm{NMR}\left(125 \mathrm{MHz}, \mathrm{CDCl}_{3}\right.$, $\left.22{ }^{\circ} \mathrm{C}\right) \delta 197.79,141.72,136.99,136.96,133.22,132.33,132.08,130.38,129.18,128.78$, $128.67,128.51,128.06,127.42,126.25,44.30,43.23$. ESI-TOF-MS $(\mathrm{m} / \mathrm{z})$ Calcd. for $\mathrm{C}_{23} \mathrm{H}_{20} \mathrm{ClO}[\mathrm{M}+\mathrm{H}]^{+}: 347.1197$; Found: $347.1201 .[\alpha]_{\mathrm{D}}^{20}=+21.33\left(c=0.05\right.$ in $\left.\mathrm{CHCl}_{3}\right)$ for a sample with $94 \%$ ee. The absolute configuration was determined by HPLC (Chiralpak IC, hexane $/ 2$-propanol $=98.5 / 1.5,1.0 \mathrm{~mL} / \mathrm{min}, \lambda=245 \mathrm{~nm}) ; t_{\mathrm{R}}=9.487 \mathrm{~min}(S$, minor $), 10.293$ $\min (R$, major).<smiles>CC(=O)CC(=Cc1ccccc1)c1cccs1</smiles>

(E)-6-phenyl-4-(thiophen-2-yl)hex-5-en-2-one (10o $)^{\mathrm{S} 13}$

According to the general procedure, the reaction of (E)-4-(thiophen-2-yl)but-3-en-2-one (8o $(5.08 \mathrm{mg})$ and $(E)$-styrylboronic acid $(\mathbf{9 a})(5.98 \mathrm{mg})$ for $72 \mathrm{~h}$ gave the adduct $\mathbf{1 0 0}(5.56 \mathrm{mg}$, 
$65 \%, 96 \%$ ee). The spectral data were consistent with the literature. ${ }^{\mathrm{S} 13}{ }^{1} \mathrm{H}$ NMR $(500 \mathrm{MHz}$, $\left.\mathrm{CDCl}_{3}, 22{ }^{\circ} \mathrm{C}\right) \delta 7.28-7.26(\mathrm{~m}, 2 \mathrm{H}), 7.23-7.20(\mathrm{~m}, 2 \mathrm{H}), 7.16-7.10(\mathrm{~m}, 2 \mathrm{H}), 6.88-6.87(\mathrm{~m}$, $1 \mathrm{H}), 6.81(\mathrm{~d}, J=3.4 \mathrm{~Hz}, 1 \mathrm{H}), 6.39(\mathrm{~d}, J=15.8 \mathrm{~Hz}, 1 \mathrm{H}), 6.25-6.20(\mathrm{~m}, 1 \mathrm{H}), 4.33-4.29(\mathrm{~m}$, 1H), 2.96-2.86 (m, 2H), 2.08 (s, 3H). ${ }^{13} \mathrm{C}$ NMR (125 MHz, $\left.\mathrm{CDCl}_{3}, 22{ }^{\circ} \mathrm{C}\right) \delta 206.25,146.77$, $136.84,131.54,130.62,128.53,127.52,126.88,126.37,124.01,123.79,50.26,39.26,30.76$. ESI-TOF-MS $(\mathrm{m} / z)$ Calcd. for $\mathrm{C}_{16} \mathrm{H}_{17} \mathrm{OS}[\mathrm{M}+\mathrm{H}]^{+}:$257.0995; Found: $257.0991 .[\alpha]_{\mathrm{D}}^{20}=+6.96$ ( $c=0.05$ in $\mathrm{CHCl}_{3}$ ) for a sample with $96 \%$ ee. The absolute configuration was determined by HPLC (Chiralpak OD-H, hexane/2-propanol $=98.5 / 1.5,1.0 \mathrm{~mL} / \mathrm{min}, \lambda=245 \mathrm{~nm}$ ); $t_{\mathrm{R}}=$ $11.827 \mathrm{~min}$ (minor), $15.967 \mathrm{~min}$ (major).<smiles>CC(=O)C[C@@H](/C=C/c1ccccc1)c1ccco1</smiles>

(E)-4-(furan-2-yl)-6-phenylhex-5-en-2-one (10p) ${ }^{\mathrm{S} 13}$

According to the general procedure, the reaction of $(E)$-4-(furan-2-yl)but-3-en-2-one $(\mathbf{8 p}$ $(4.54 \mathrm{mg})$ and $(E)$-styrylboronic acid $(\mathbf{9 a})(5.98 \mathrm{mg})$ for $72 \mathrm{~h}$ gave the adduct $\mathbf{1 0 p}(4.49 \mathrm{mg}$, $56 \%, 94 \%$ ee). The spectral data were consistent with the literature. ${ }^{\mathrm{S} 13}{ }^{1} \mathrm{H}$ NMR $(500 \mathrm{MHz}$, $\left.\mathrm{CDCl}_{3}, 22^{\circ} \mathrm{C}\right) \delta 7.28-7.26(\mathrm{~m}, 3 \mathrm{H}), 7.22(\mathrm{t}, J=7.6 \mathrm{~Hz}, 2 \mathrm{H}), 7.14(\mathrm{t}, J=7.2 \mathrm{~Hz}, 1 \mathrm{H}), 6.37(\mathrm{~d}$, $J=15.9 \mathrm{~Hz}, 1 \mathrm{H}), 6.23-6.22(\mathrm{~m}, 1 \mathrm{H}), 6.19-6.15(\mathrm{~m}, 1 \mathrm{H}), 6.01(\mathrm{~d}, J=3.2 \mathrm{~Hz}, 1 \mathrm{H}), 4.12-4.08$ (m, 1H), 2.97-2.76 (m, 2H), $2.08(\mathrm{~s}, 3 \mathrm{H}) .{ }^{13} \mathrm{C}$ NMR (125 MHz, $\left.\mathrm{CDCl}_{3}, 22{ }^{\circ} \mathrm{C}\right) \delta 205.30$, 154.72, 140.50, 135.86, 130.32, 128.13, 127.49, 126.47, 125.31, 109.24, 104.41, 46.22, 36.76, 29.50. ESI-TOF-MS $(m / z)$ Calcd. for $\mathrm{C}_{16} \mathrm{H}_{17} \mathrm{O}_{2}[\mathrm{M}+\mathrm{H}]^{+}:$241.1223; Found: 241.1221. $[\alpha]_{\mathrm{D}}^{20}=$ $-42.67\left(c=0.05\right.$ in $\left.\mathrm{CHCl}_{3}\right)$ for a sample with $94 \%$ ee. The absolute configuration was determined by HPLC (Chiralpak OD-H, hexane/2-propanol $=98.5 / 1.5,1.0 \mathrm{~mL} / \mathrm{min}, \lambda=245$ $\mathrm{nm}) ; t_{\mathrm{R}}=9.358 \min$ (minor), $11.491 \min$ (major). 
<smiles>COc1ccc(C(C=Cc2ccc(F)cc2)CC(=O)c2ccccc2)cc1</smiles>

(E)-5-(4-fluorophenyl)-3-(4-methoxyphenyl)-1-phenylpent-4-en-1-one (10q)

According to the general procedure, the reaction of (E)-3-(4-methoxyphenyl)-1-phenylprop-2-en-1-one $\quad$ (81) $\quad(7.95 \quad \mathrm{mg}) \quad$ and trans-2-(4-fluorophenyl)vinylboronic acid (9b) $(6.64 \mathrm{mg})$ for $40 \mathrm{~h}$ gave the adduct 10q (11.42 mg, 95\%, 93\% ee). ${ }^{1} \mathrm{H}$ NMR $\left(500 \mathrm{MHz}, \mathrm{CDCl}_{3}, 22{ }^{\circ} \mathrm{C}\right) \delta 7.88-7.83(\mathrm{~m}, 2 \mathrm{H}), 7.47(\mathrm{t}, J=7.4$ $\mathrm{Hz}, 1 \mathrm{H}), 7.36$ (t, $J=7.7 \mathrm{~Hz}, 2 \mathrm{H}), 7.18-7.13(\mathrm{~m}, 4 \mathrm{H}), 6.88-6.84(\mathrm{~m}, 2 \mathrm{H}), 6.79$ (t, $J=5.8 \mathrm{~Hz}$, 2H), 6.27-6.18 (m, 2H), 4.18-4.14 (m, 1H), $3.70(\mathrm{~s}, 3 \mathrm{H}), 3.43-3.33(\mathrm{~m}, 2 \mathrm{H}) .{ }^{13} \mathrm{C}$ NMR $(125$ $\left.\mathrm{MHz}, \mathrm{CDCl}_{3}, 22{ }^{\circ} \mathrm{C}\right) \delta 198.28,162.10\left(\mathrm{~d}, J_{\mathrm{F}-\mathrm{C}}=245 \mathrm{~Hz}\right), 158.32,137.16,135.23,133.42(\mathrm{~d}$, $\left.J_{\mathrm{F}-\mathrm{C}}=3.1 \mathrm{~Hz}\right), 133.11,132.76,132.74,128.71,128.65,128.11,127.70\left(\mathrm{~d}, J_{\mathrm{F}-\mathrm{C}}=7.8 \mathrm{~Hz}\right)$, $115.32\left(\mathrm{~d}, J_{\mathrm{F}-\mathrm{C}}=21.4 \mathrm{~Hz}\right), 114.12,55.28,44.64,43.12$. EI-TOF-MS $(\mathrm{m} / \mathrm{z})$ Calcd. for $\mathrm{C}_{24} \mathrm{H}_{21} \mathrm{FO}_{2}[\mathrm{M}]^{+}:$360.1526; Found: 360.1528. $[\alpha]_{\mathrm{D}}^{20}=+2.47\left(c=0.05\right.$ in $\left.\mathrm{CHCl}_{3}\right)$ for a sample with 93\% ee. The absolute configuration was determined by HPLC (Chiralpak AS-H, hexane/2-propanol $=97 / 3,1.0 \mathrm{~mL} / \mathrm{min}, \lambda=245 \mathrm{~nm}$ ); $t_{\mathrm{R}}=18.259 \mathrm{~min}($ minor), $20.928 \mathrm{~min}$ (major).<smiles>COc1ccc(C(C=Cc2ccc(C)cc2)CC(=O)c2ccccc2)cc1</smiles>

(E)-3-(4-methoxyphenyl)-1-phenyl-5-(p-tolyl)pent-4-en-1-one (10r)

According to the general procedure, the reaction of (E)-3-(4-methoxyphenyl)-1-phenylprop-2-en-1-one $\quad(\mathbf{8 1}) \quad(7.95 \quad \mathrm{mg}) \quad$ and trans-2-(4-methylphenyl)vinylboronic acid (9c) $(6.48 \mathrm{mg})$ for $40 \mathrm{~h}$ gave the adduct $10 \mathrm{r}(11.65$ 
$\mathrm{mg}, 98 \%, 94 \%$ ee). ${ }^{1} \mathrm{H} \mathrm{NMR}\left(500 \mathrm{MHz}, \mathrm{CDCl}_{3}, 22{ }^{\circ} \mathrm{C}\right) \delta 7.85(\mathrm{~d}, J=7.4 \mathrm{~Hz}, 2 \mathrm{H}), 7.46(\mathrm{t}, J=$ $7.1 \mathrm{~Hz}, 1 \mathrm{H}), 7.36(\mathrm{t}, J=7.7 \mathrm{~Hz}, 2 \mathrm{H}), 7.17-7.10(\mathrm{~m}, 4 \mathrm{H}), 6.98(\mathrm{~d}, J=8.0 \mathrm{~Hz}, 2 \mathrm{H}), 6.77(\mathrm{~d}, J=$ $8.5 \mathrm{~Hz}, 2 \mathrm{H}), 6.26(\mathrm{~d}, J=3.6 \mathrm{~Hz}, 2 \mathrm{H}), 4.16(\mathrm{~d}, J=4.0 \mathrm{~Hz}, 1 \mathrm{H}), 3.69(\mathrm{~s}, 3 \mathrm{H}), 3.43-3.31(\mathrm{~m}$, 2H), $2.22(\mathrm{~s}, 3 \mathrm{H}) .{ }^{13} \mathrm{C} \mathrm{NMR}\left(125 \mathrm{MHz}, \mathrm{CDCl}_{3}, 22{ }^{\circ} \mathrm{C}\right) \delta 198.41,158.25,137.25,136.97$, $135.46,134.49,133.04,131.94,129.62,129.16,128.76,128.62,128.12,126.15,114.05$, 55.28, 44.74, 43.17, 21.17. EI-TOF-MS (m/z) Calcd. for $\mathrm{C}_{25} \mathrm{H}_{24} \mathrm{O}_{2}[\mathrm{M}]^{+}:$356.1776; Found: 356.1776. $[\alpha]_{\mathrm{D}}^{20}=+2.13\left(c=0.05\right.$ in $\left.\mathrm{CHCl}_{3}\right)$ for a sample with $94 \%$ ee. The absolute configuration was determined by HPLC (Chiralpak OD-H, hexane/2-propanol = 97/3, 1.0 $\mathrm{mL} / \mathrm{min}, \lambda=245 \mathrm{~nm}$ ); $t_{\mathrm{R}}=12.645 \min$ (minor), $14.382 \min$ (major). 
9. ${ }^{1} \mathrm{H}$ and ${ }^{13} \mathrm{C}$ NMR spectra and chiral HPLC spectra of the addition products

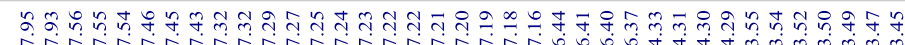

rivis
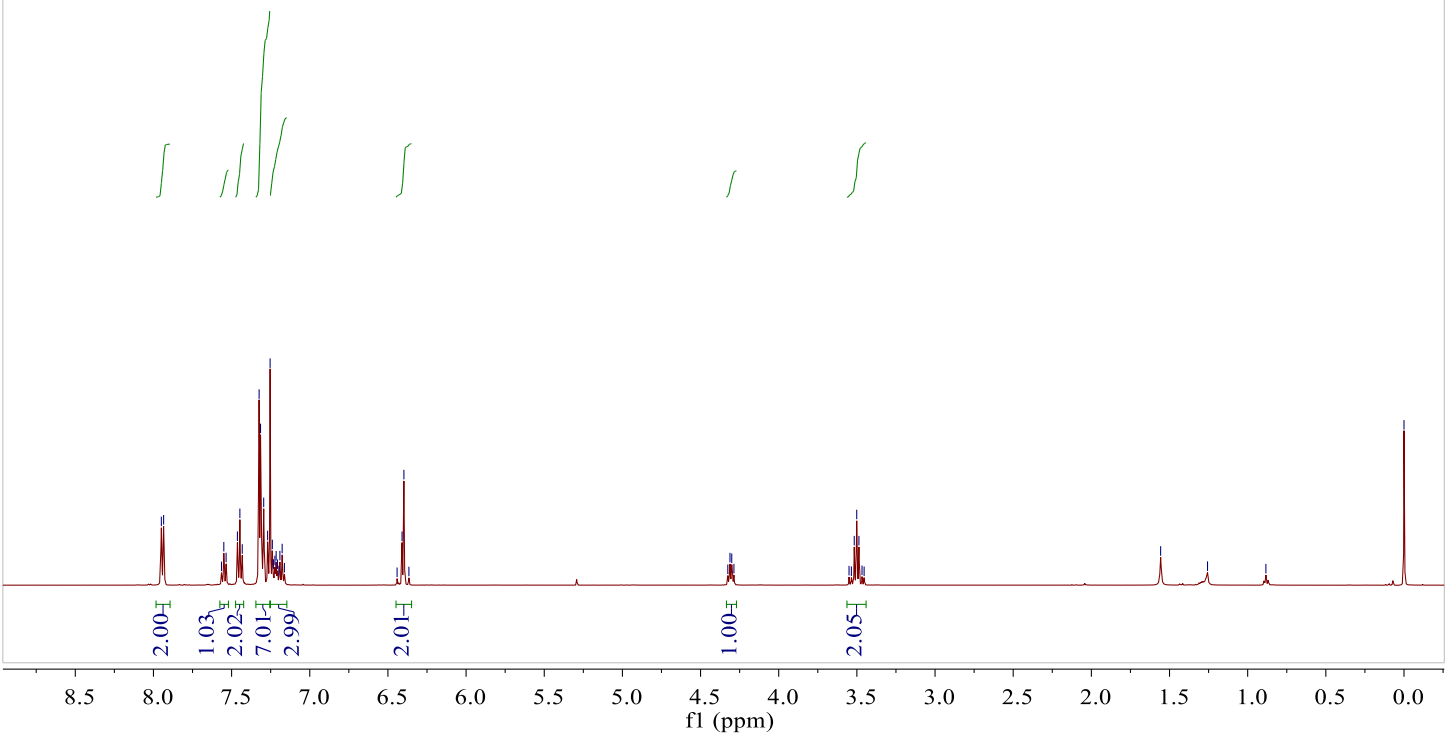

Figure S22. ${ }^{1} \mathrm{H}$ NMR $\left(500 \mathrm{MHz}, \mathrm{CDCl}_{3}, 22{ }^{\circ} \mathrm{C}\right)$ spectrum of $\mathbf{1 0 a}$.

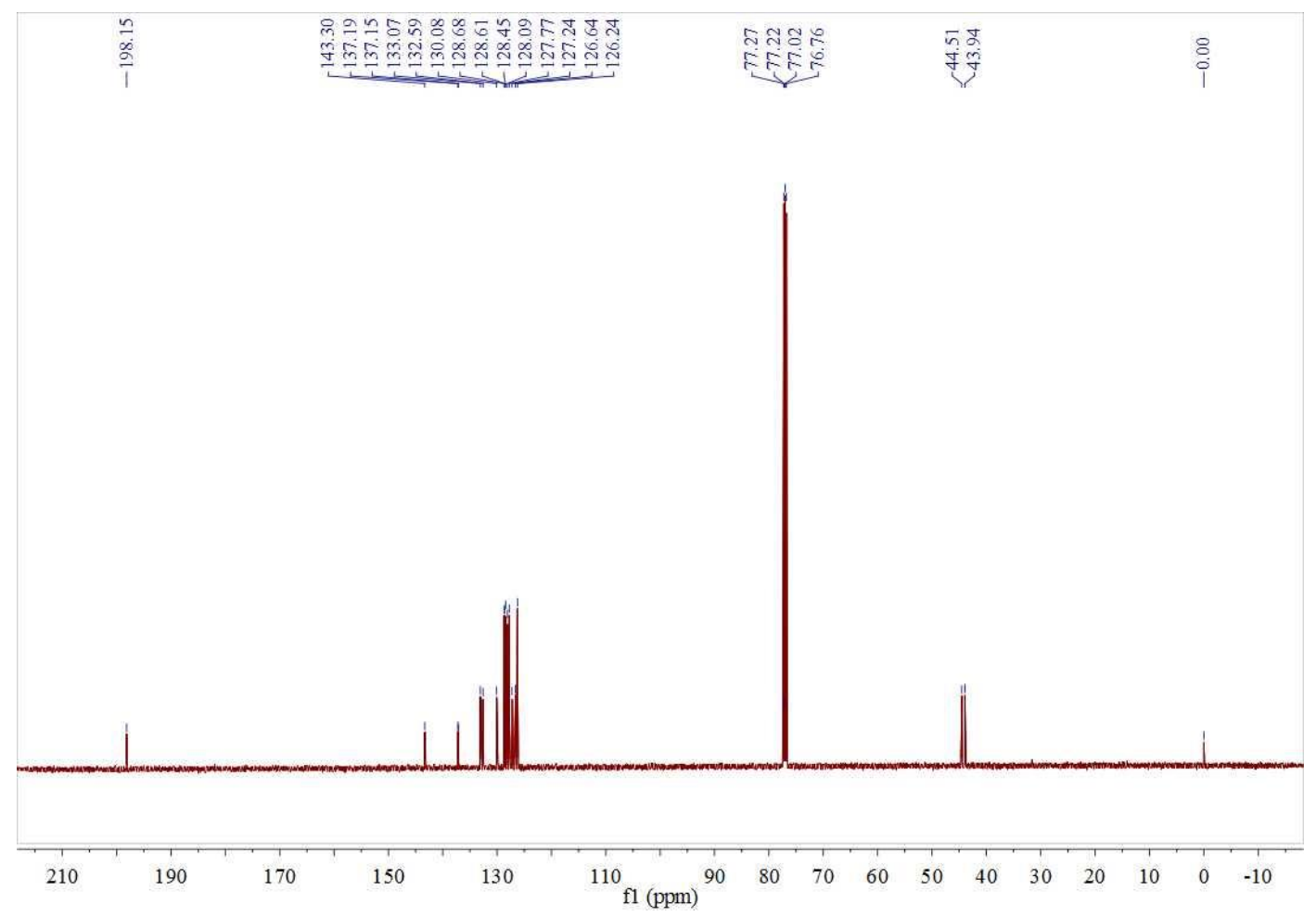

Figure S23. ${ }^{13} \mathrm{C}$ NMR $\left(125 \mathrm{MHz}, \mathrm{CDCl}_{3}, 22{ }^{\circ} \mathrm{C}\right)$ spectrum of 10a. 

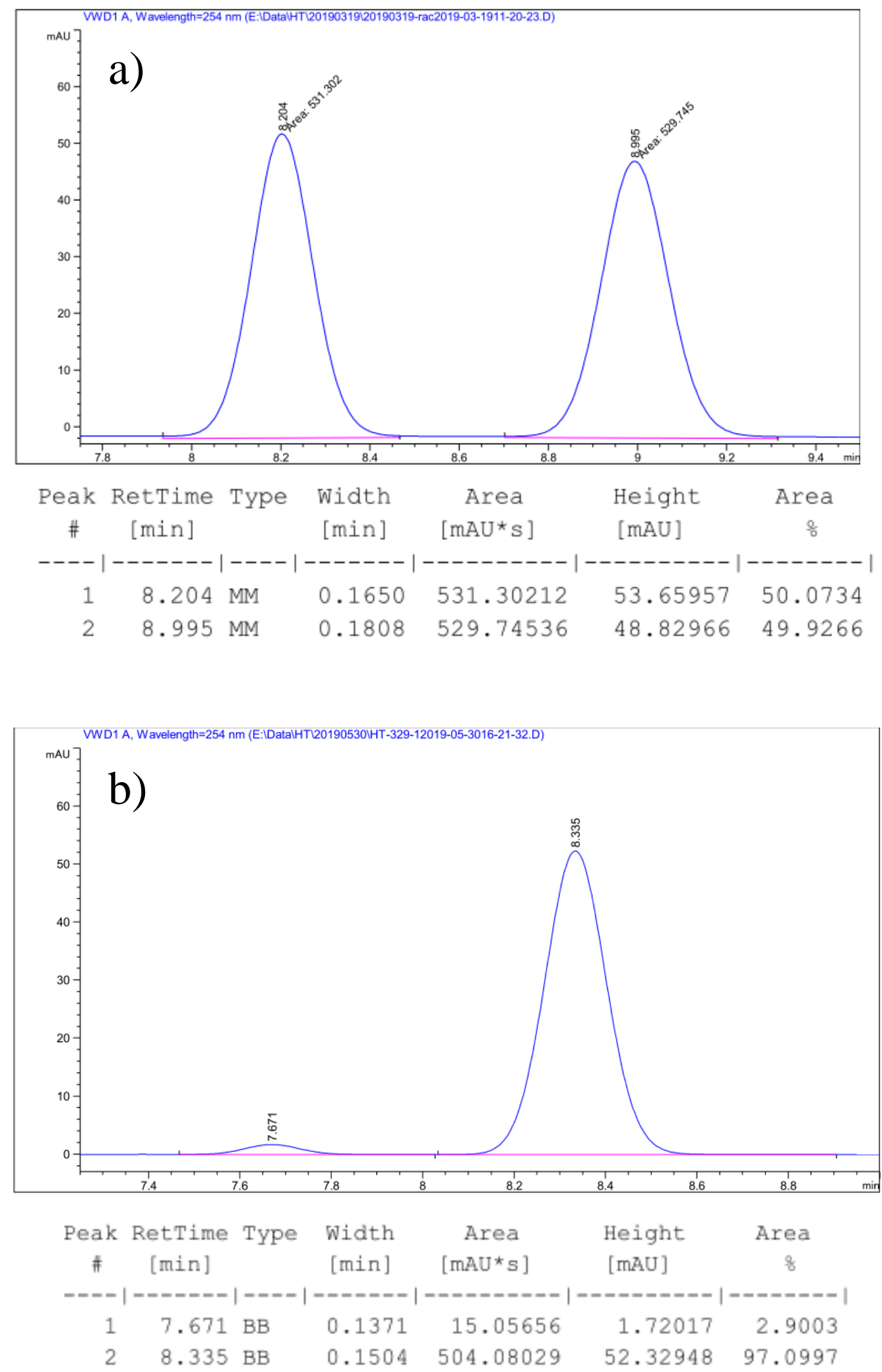

Figure S24. Chiral HPLC analysis of a) racemic 10a and b) (R)-10a (Chiralpak IC, hexane/2-propanol $=98.5 / 1.5,1.0 \mathrm{~mL} / \mathrm{min}, \lambda=254 \mathrm{~nm}$ ). 


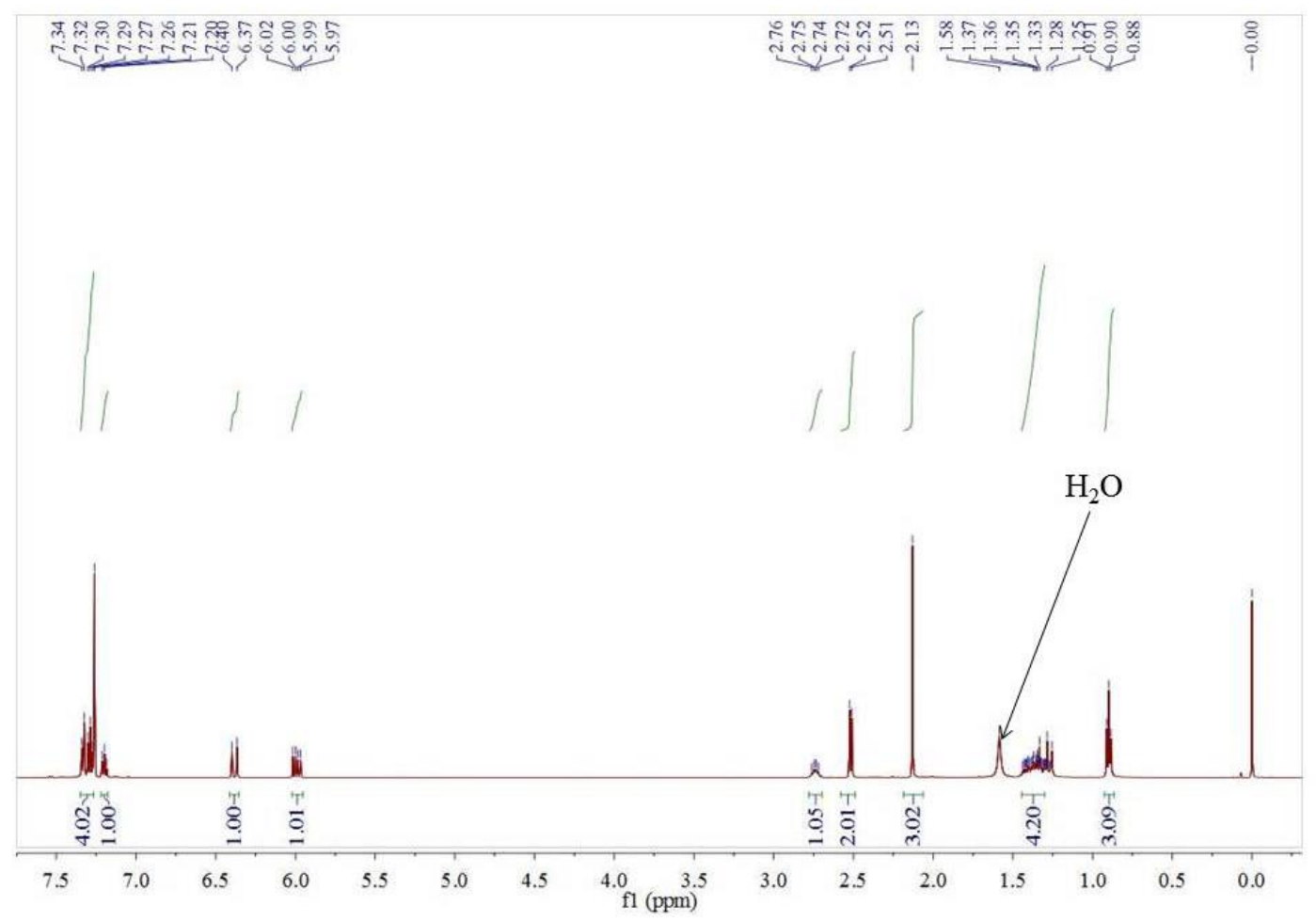

Figure S25. ${ }^{1} \mathrm{H}$ NMR $\left(500 \mathrm{MHz}, \mathrm{CDCl}_{3}, 22{ }^{\circ} \mathrm{C}\right)$ spectrum of $\mathbf{1 0 b}$.

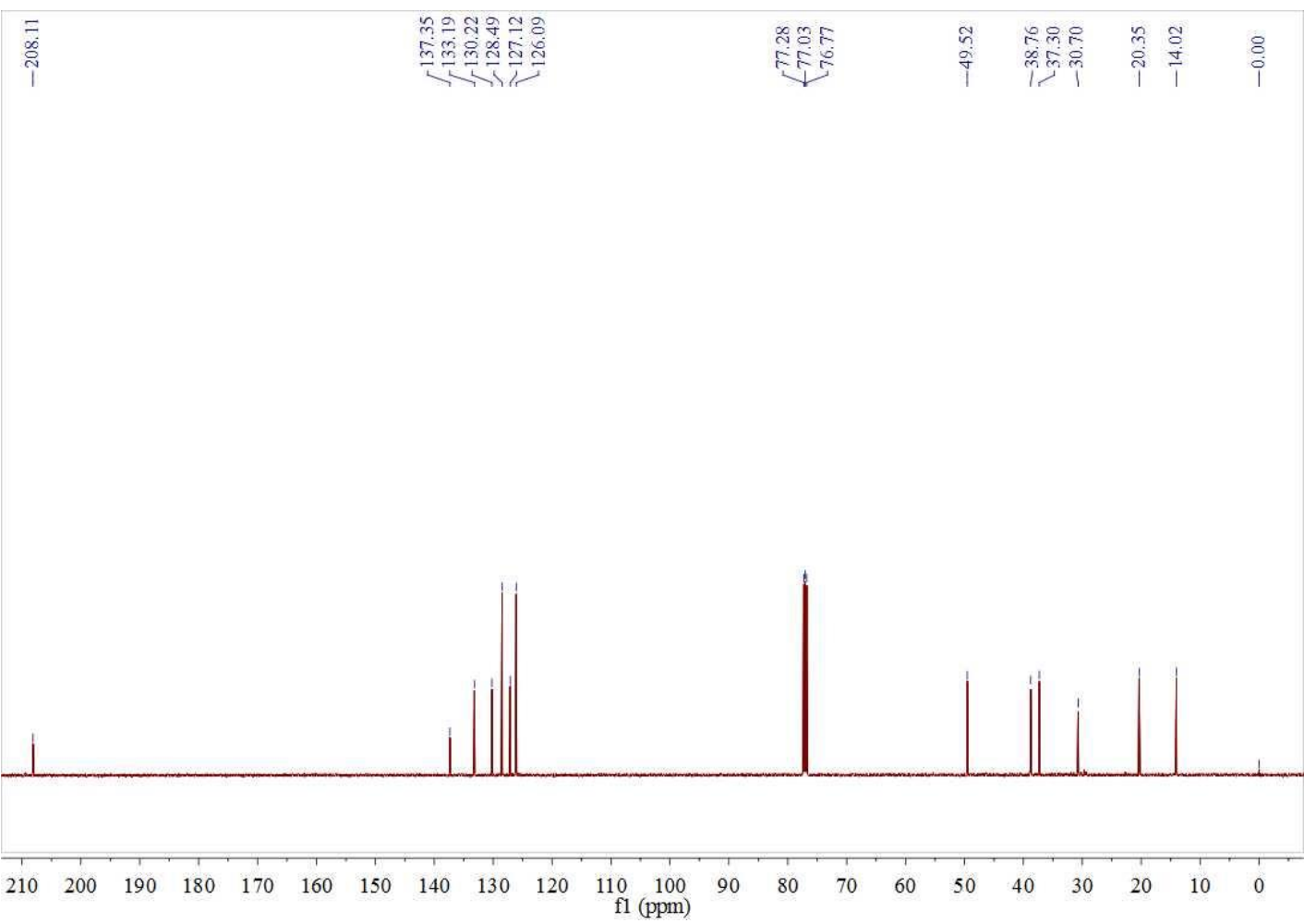

Figure S26. ${ }^{13} \mathrm{C}$ NMR $\left(125 \mathrm{MHz}, \mathrm{CDCl}_{3}, 22{ }^{\circ} \mathrm{C}\right)$ spectrum of $\mathbf{1 0 b}$. 

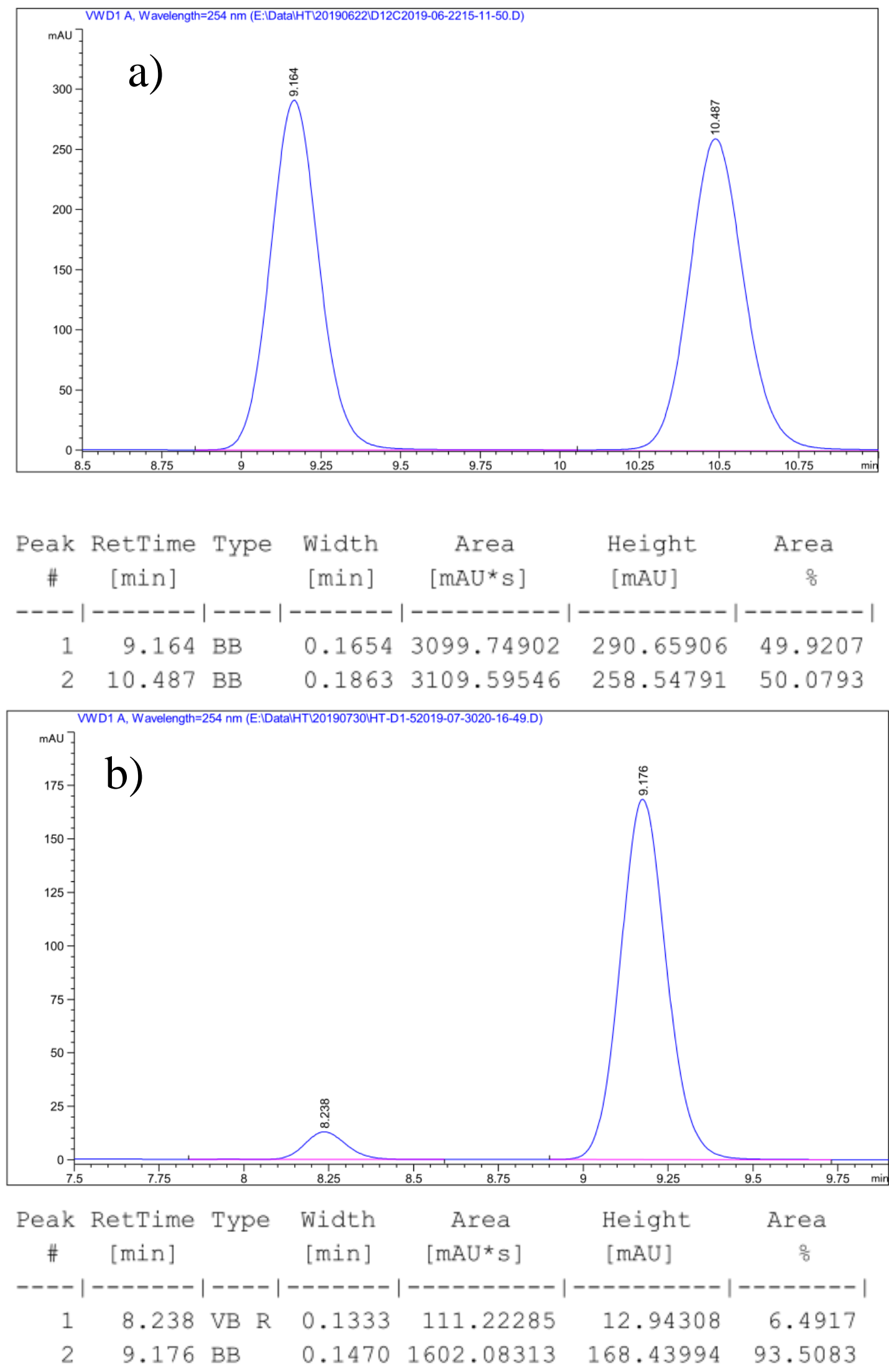

Figure S27. Chiral HPLC analysis of a) racemic 10b and b) $(R)-\mathbf{1 0 b}$ (Chiralpak AD-H hexane/2-propanol $=99.5 / 0.5,1.0 \mathrm{~mL} / \mathrm{min}, \lambda=254 \mathrm{~nm}$ ). 


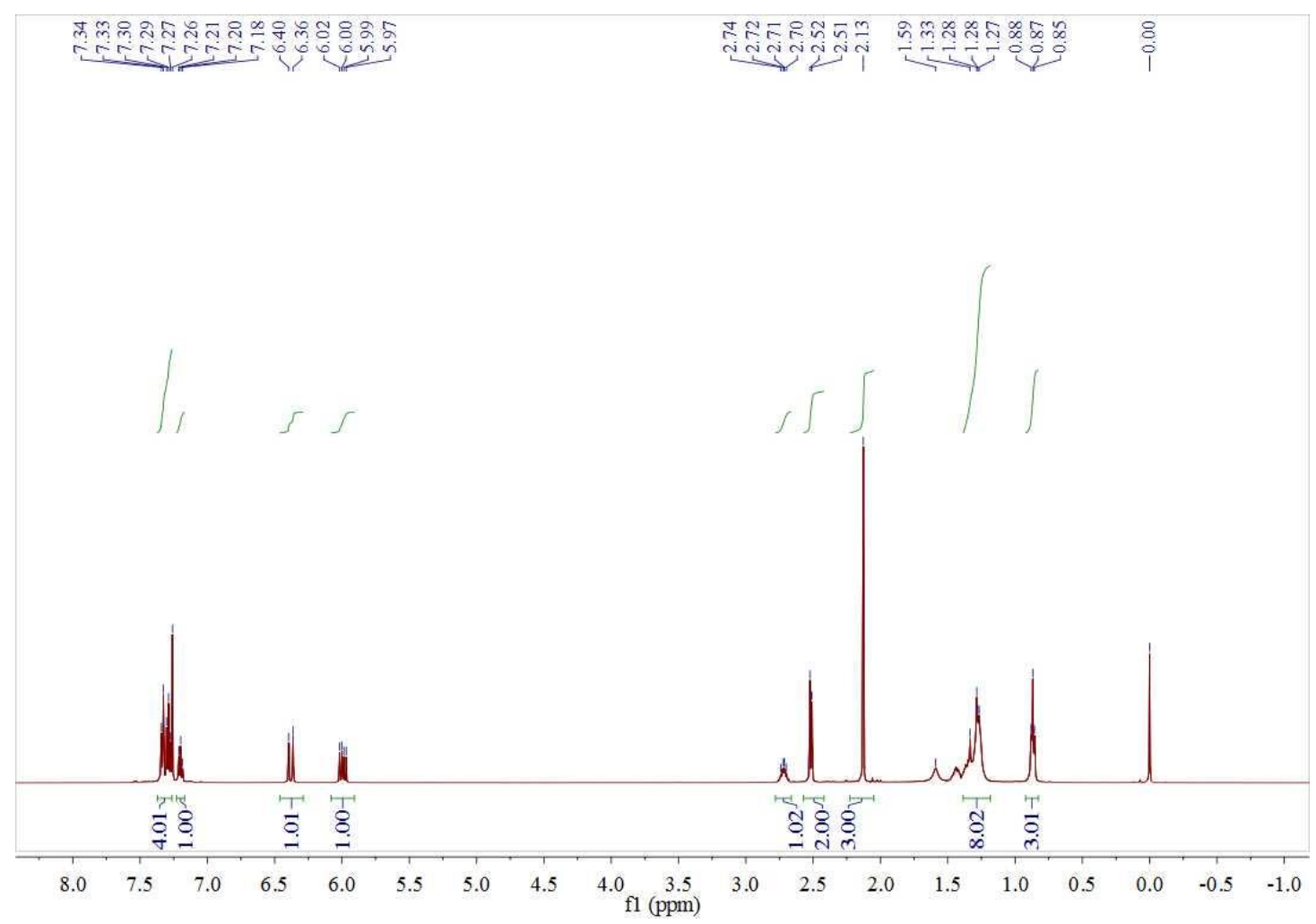

Figure S28. ${ }^{1} \mathrm{H}$ NMR $\left(500 \mathrm{MHz}, \mathrm{CDCl}_{3}, 22{ }^{\circ} \mathrm{C}\right)$ spectrum of $\mathbf{1 0 c}$.

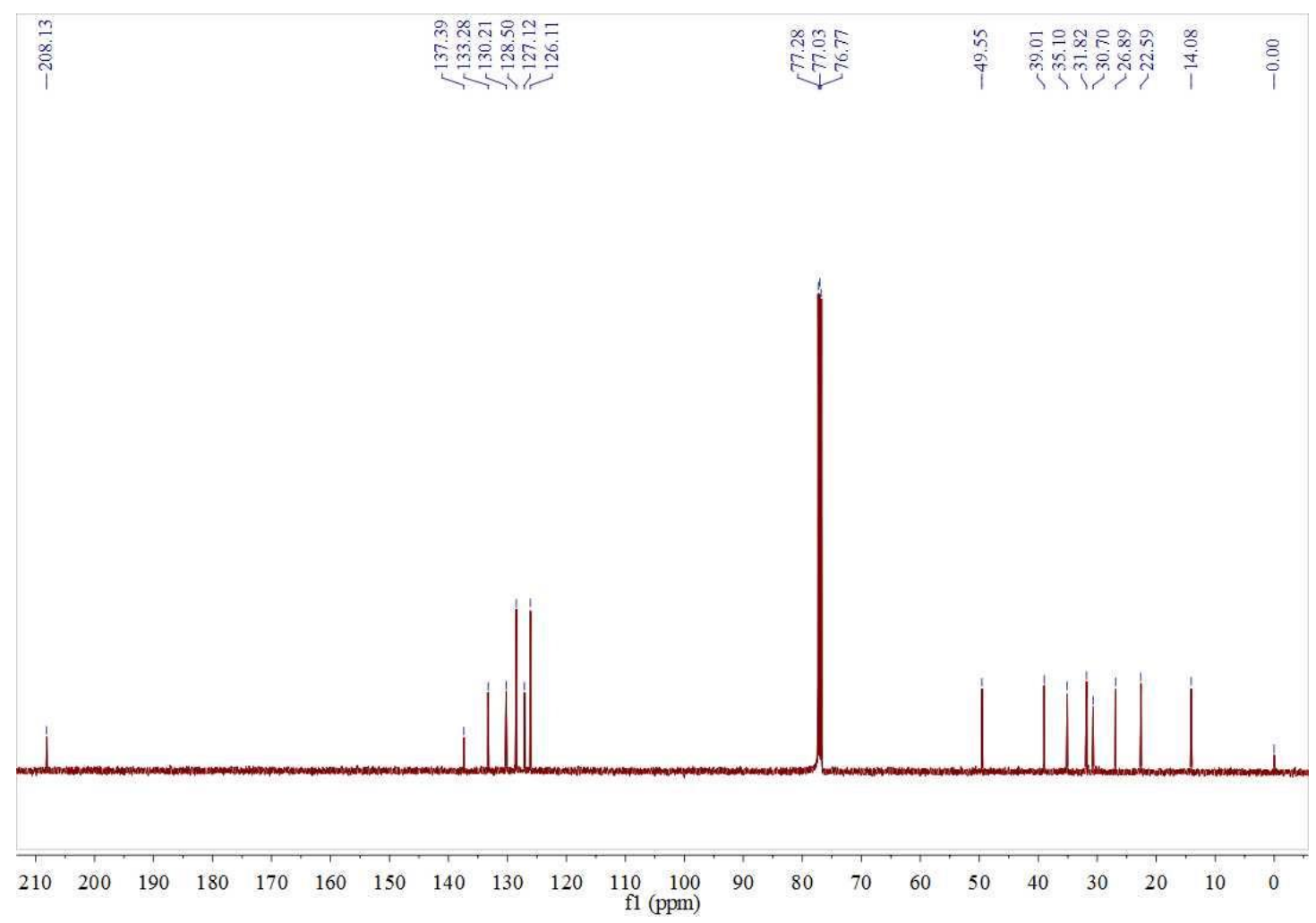

Figure S29. ${ }^{13} \mathrm{C}$ NMR $\left(125 \mathrm{MHz}, \mathrm{CDCl}_{3}, 22{ }^{\circ} \mathrm{C}\right)$ spectrum of $\mathbf{1 0 c}$. 

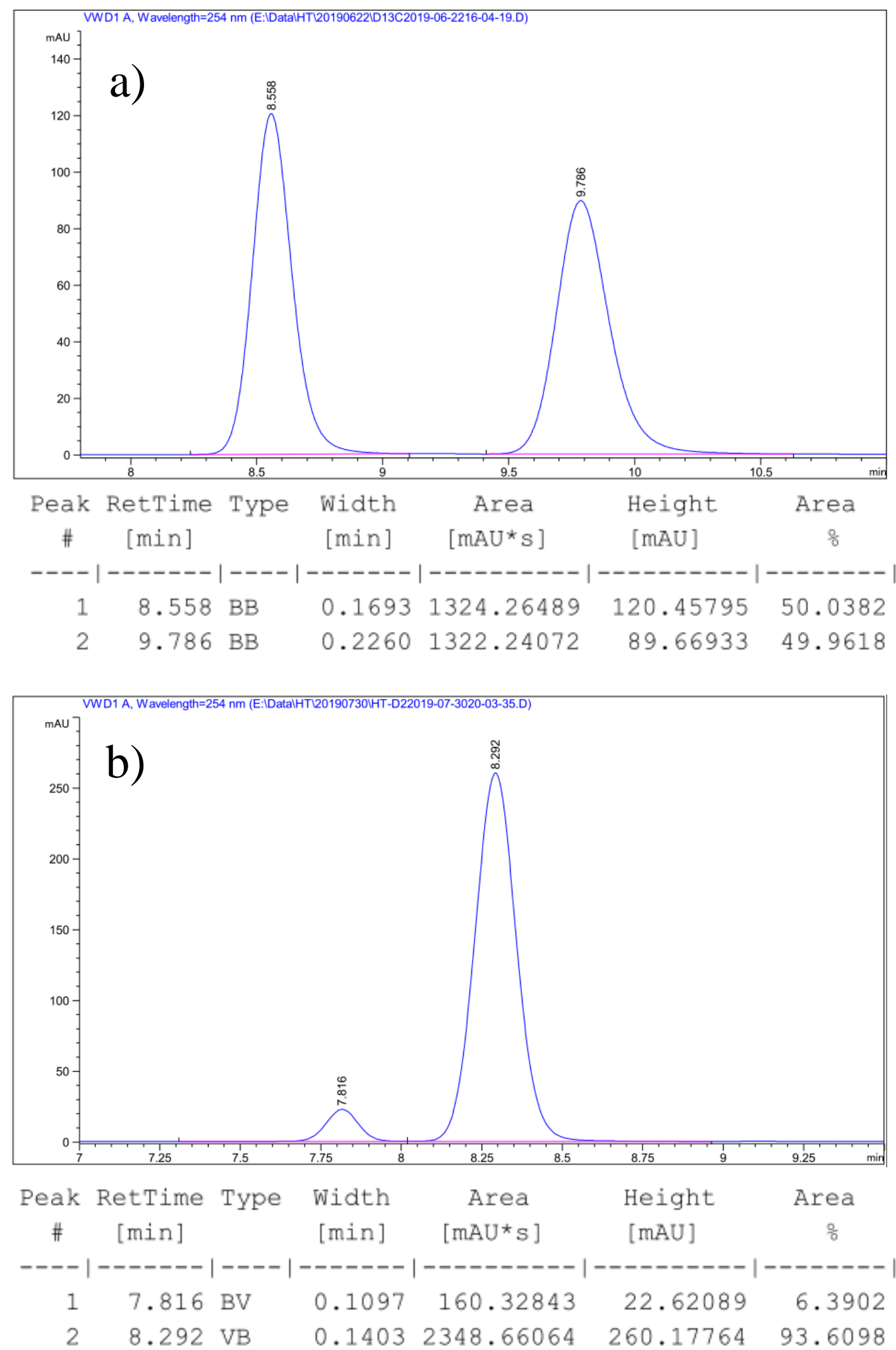

Figure S30. Chiral HPLC analysis of a) racemic 10c and b) (-)-10c (Chiralpak AD-H hexane/2-propanol 99.5/0.5, $1.0 \mathrm{~mL} / \mathrm{min}, \lambda=254 \mathrm{~nm}$ ). 


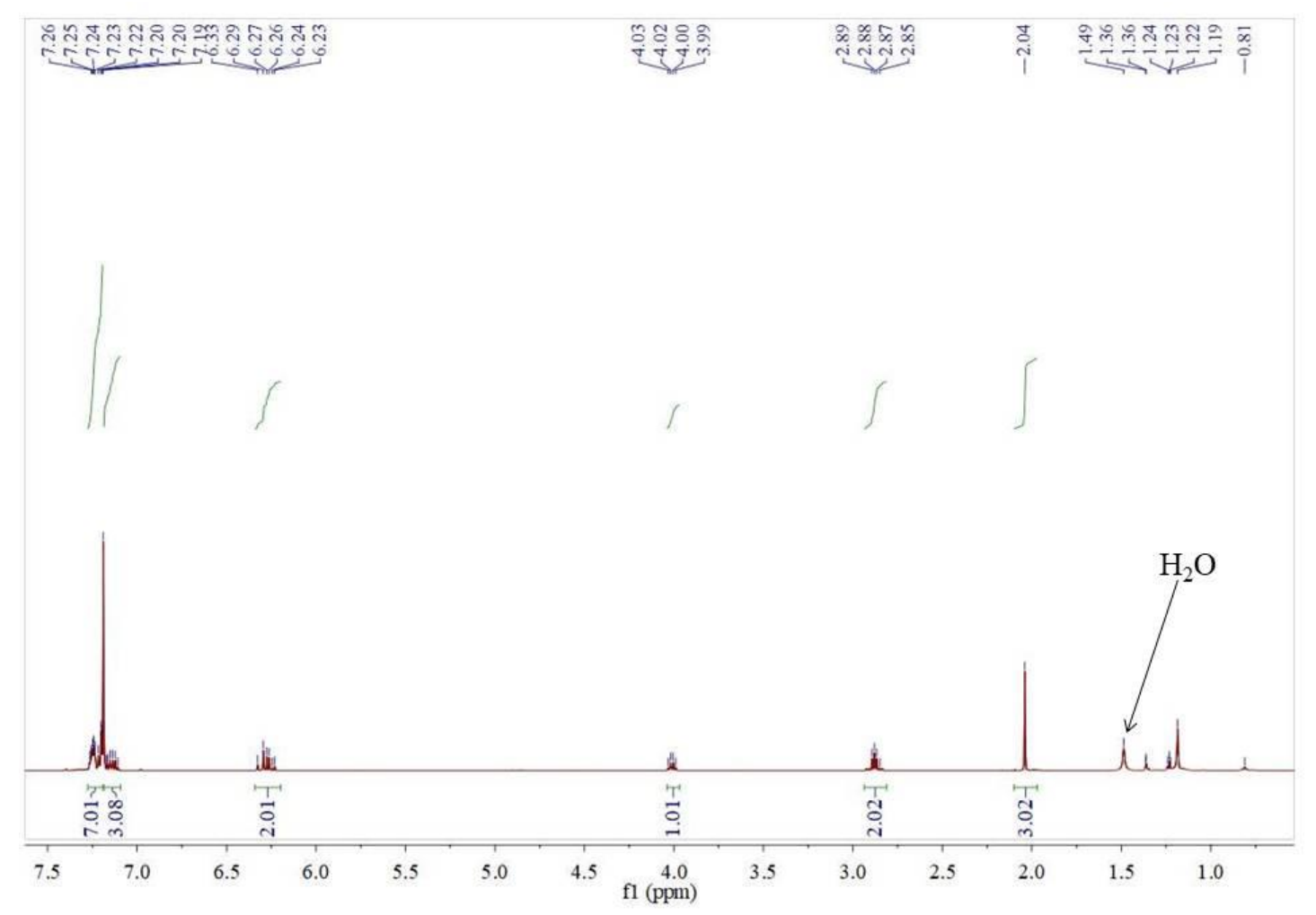

Figure S31. ${ }^{1} \mathrm{H}$ NMR $\left(500 \mathrm{MHz}, \mathrm{CDCl}_{3}, 22{ }^{\circ} \mathrm{C}\right)$ spectrum of $\mathbf{1 0 d}$.

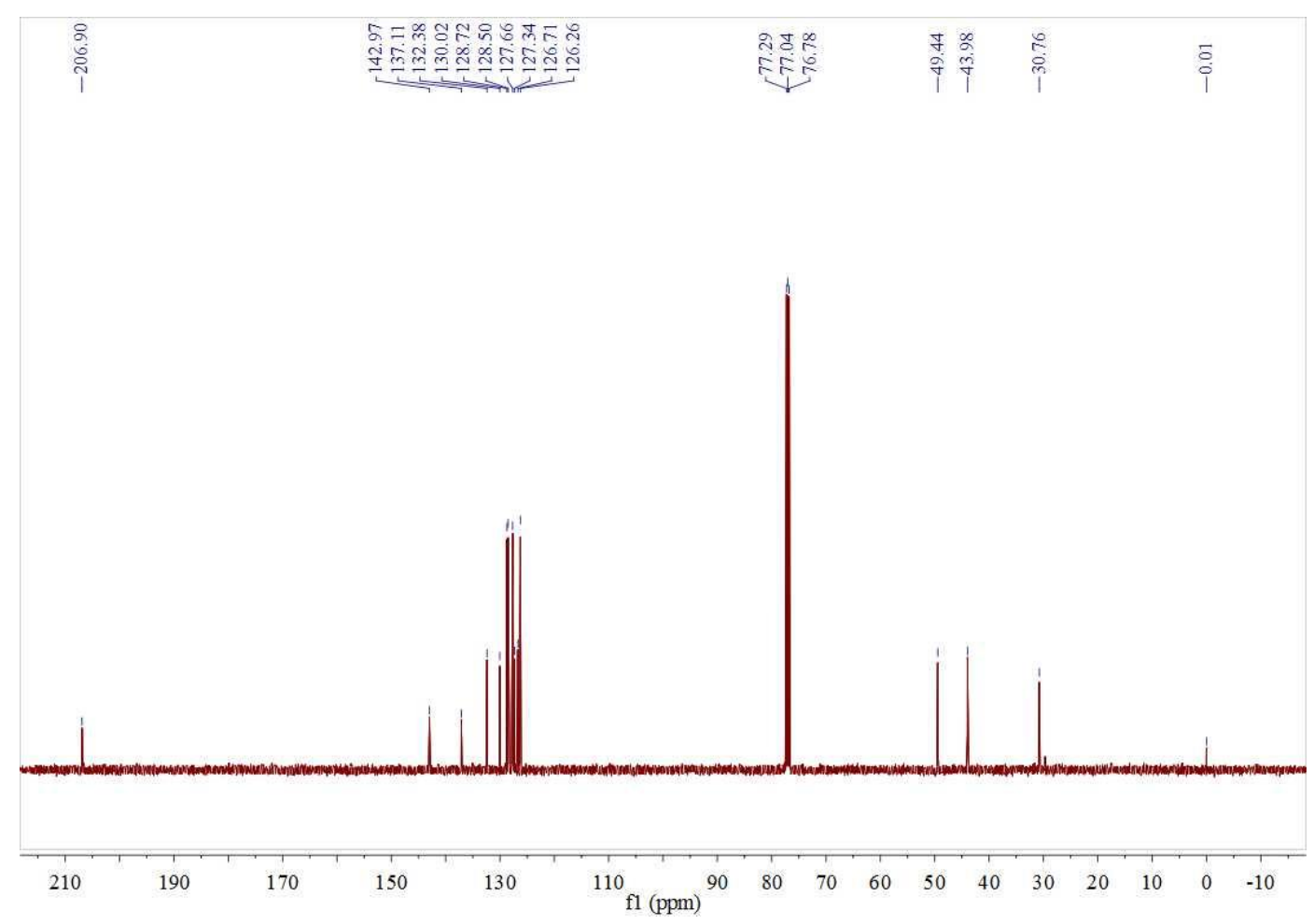

Figure S32. ${ }^{13} \mathrm{C}$ NMR $\left(125 \mathrm{MHz}, \mathrm{CDCl}_{3}, 22{ }^{\circ} \mathrm{C}\right)$ spectrum of $\mathbf{1 0 d}$. 

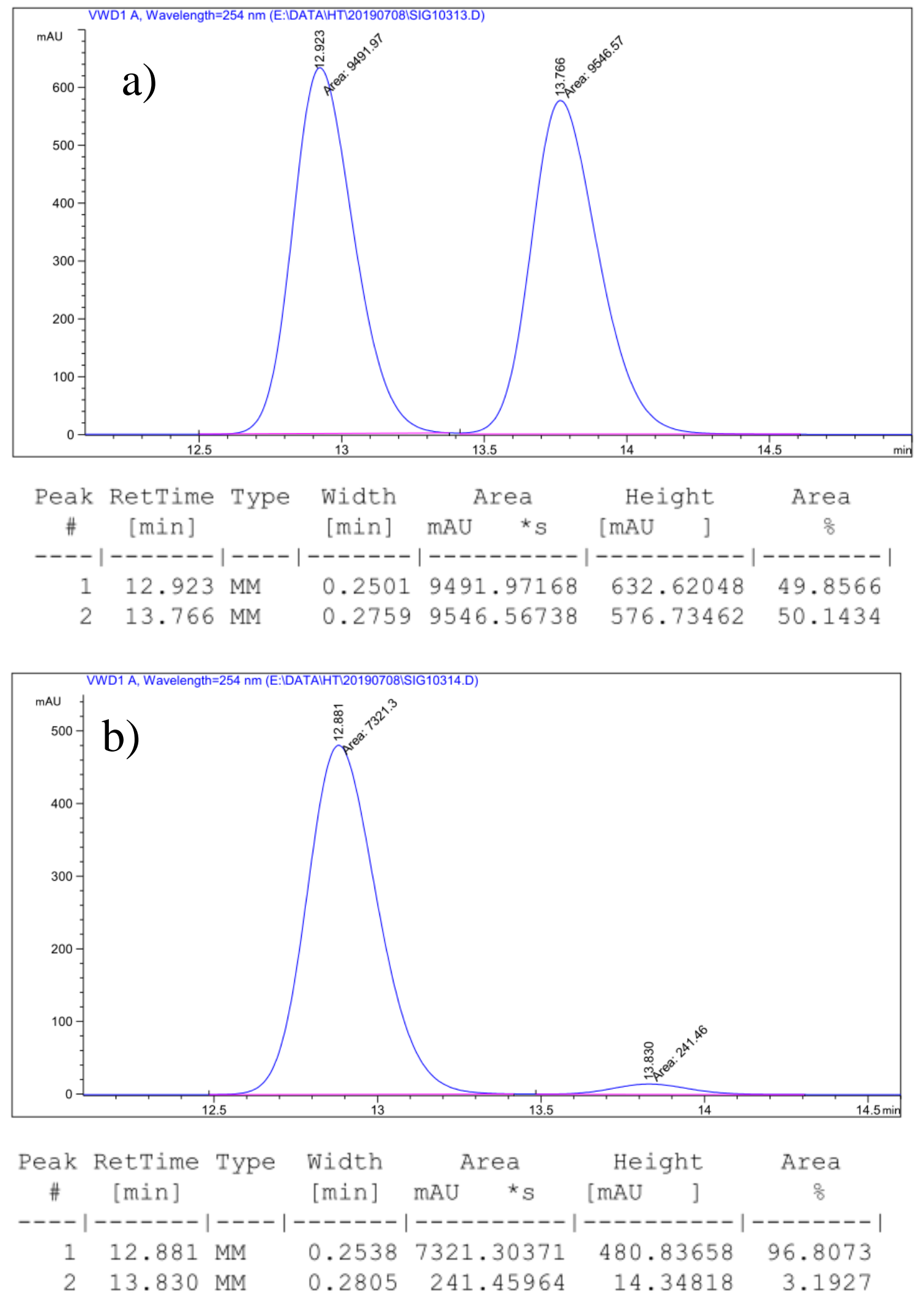

Figure S33. Chiral HPLC analysis of a) racemic 10d and b) $(R)$-10d (Chiralpak AD-H hexane/2-propanol 98.5/1.5, $1.0 \mathrm{~mL} / \mathrm{min}, \lambda=254 \mathrm{~nm}$ ). 


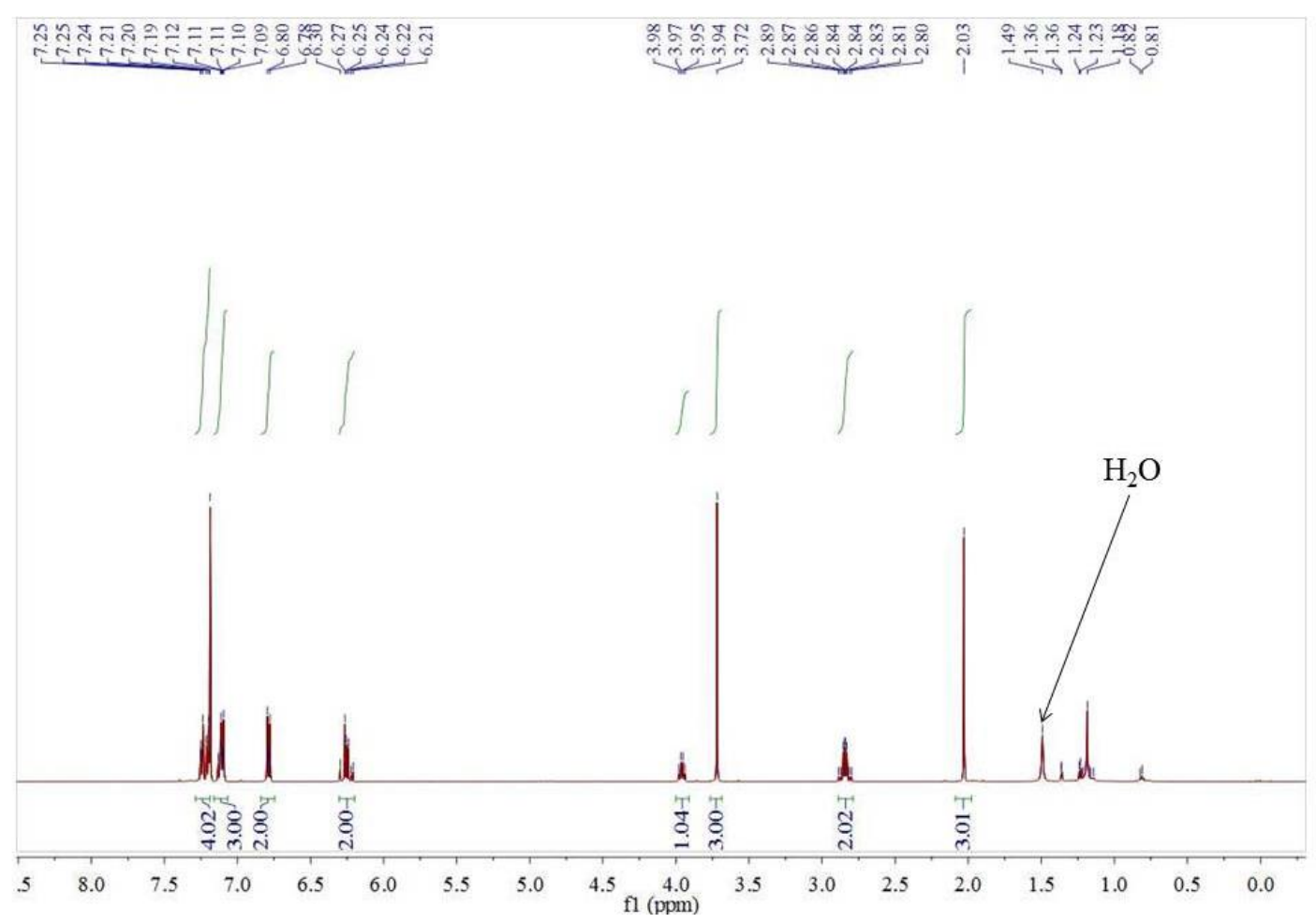

Figure S34. ${ }^{1} \mathrm{H}$ NMR $\left(500 \mathrm{MHz}, \mathrm{CDCl}_{3}, 22{ }^{\circ} \mathrm{C}\right)$ spectrum of $\mathbf{1 0 e}$.

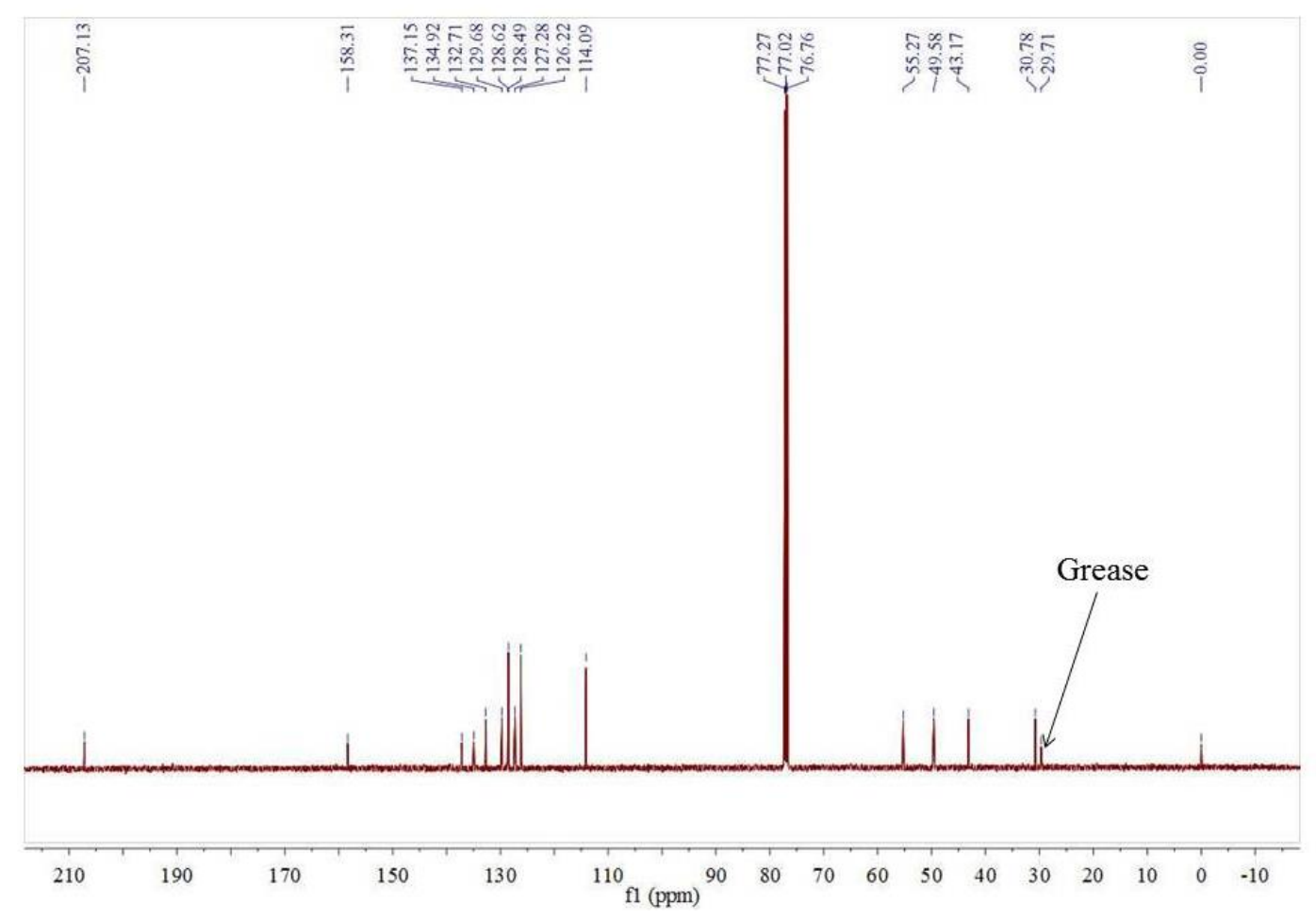

Figure S35. ${ }^{13} \mathrm{C}$ NMR $\left(125 \mathrm{MHz}, \mathrm{CDCl}_{3}, 22{ }^{\circ} \mathrm{C}\right)$ spectrum of $\mathbf{1 0 e}$. 

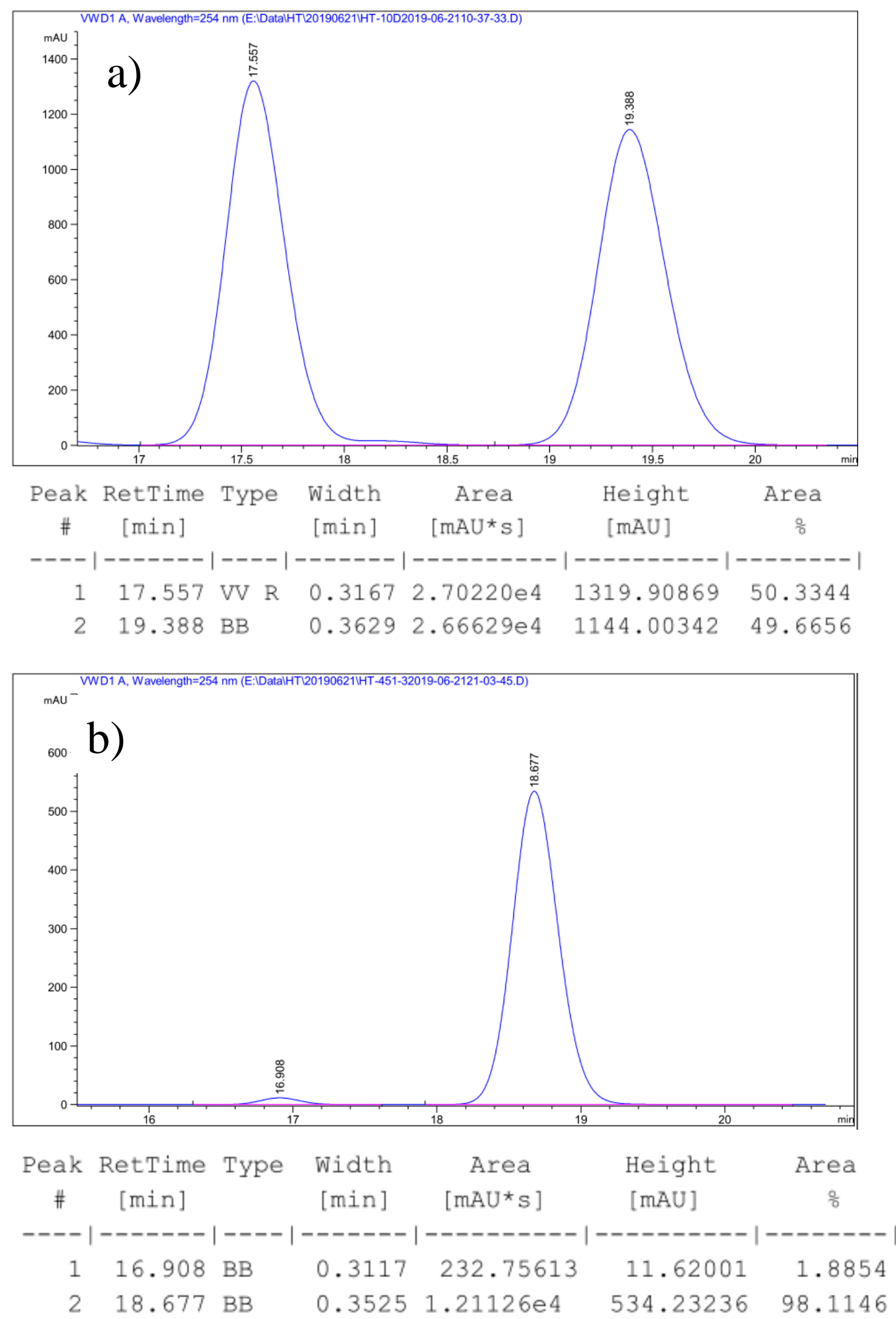

Figure S36. Chiral HPLC analysis of a) racemic 10e and b) (+)-10e (Chiralpak AD-H hexane/2-propanol 98.5/1.5, $1.0 \mathrm{~mL} / \mathrm{min}, \lambda=254 \mathrm{~nm}$ ). 


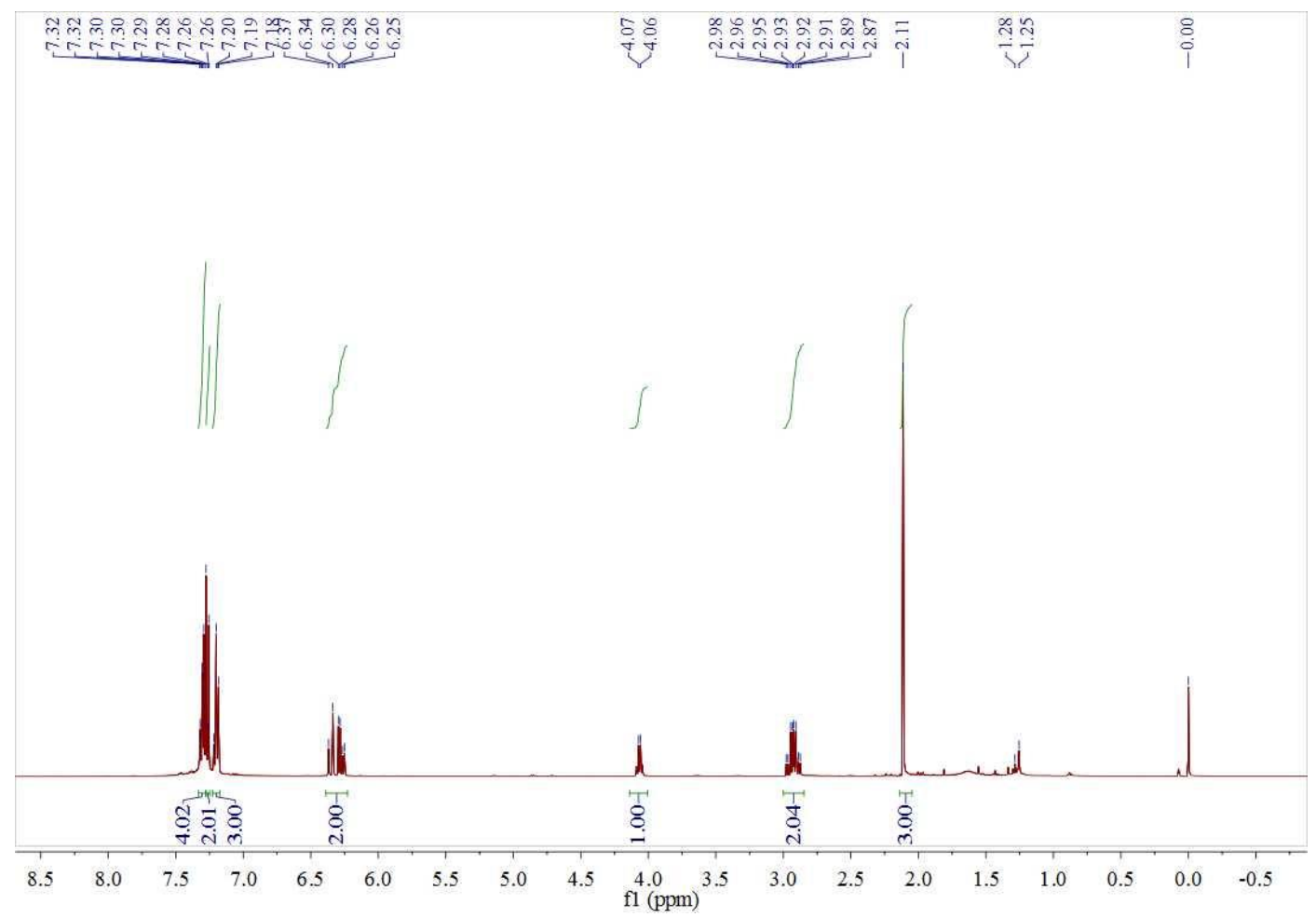

Figure S37. ${ }^{1} \mathrm{H}$ NMR $\left(500 \mathrm{MHz}, \mathrm{CDCl}_{3}, 22{ }^{\circ} \mathrm{C}\right)$ spectrum of $\mathbf{1 0 f}$.

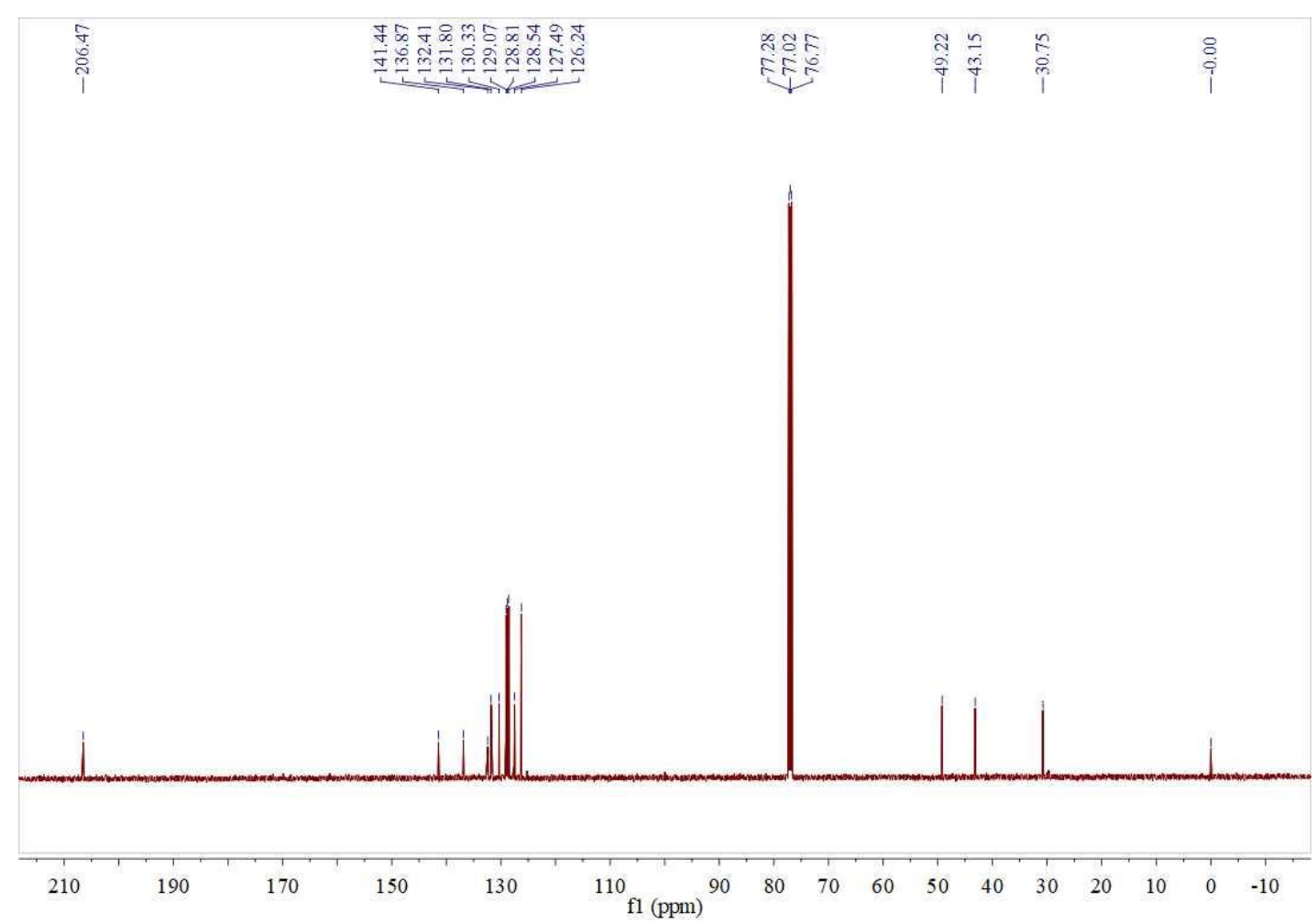

Figure S38. ${ }^{13} \mathrm{C}$ NMR $\left(125 \mathrm{MHz}, \mathrm{CDCl}_{3}, 22{ }^{\circ} \mathrm{C}\right)$ spectrum of $\mathbf{1 0 f}$. 

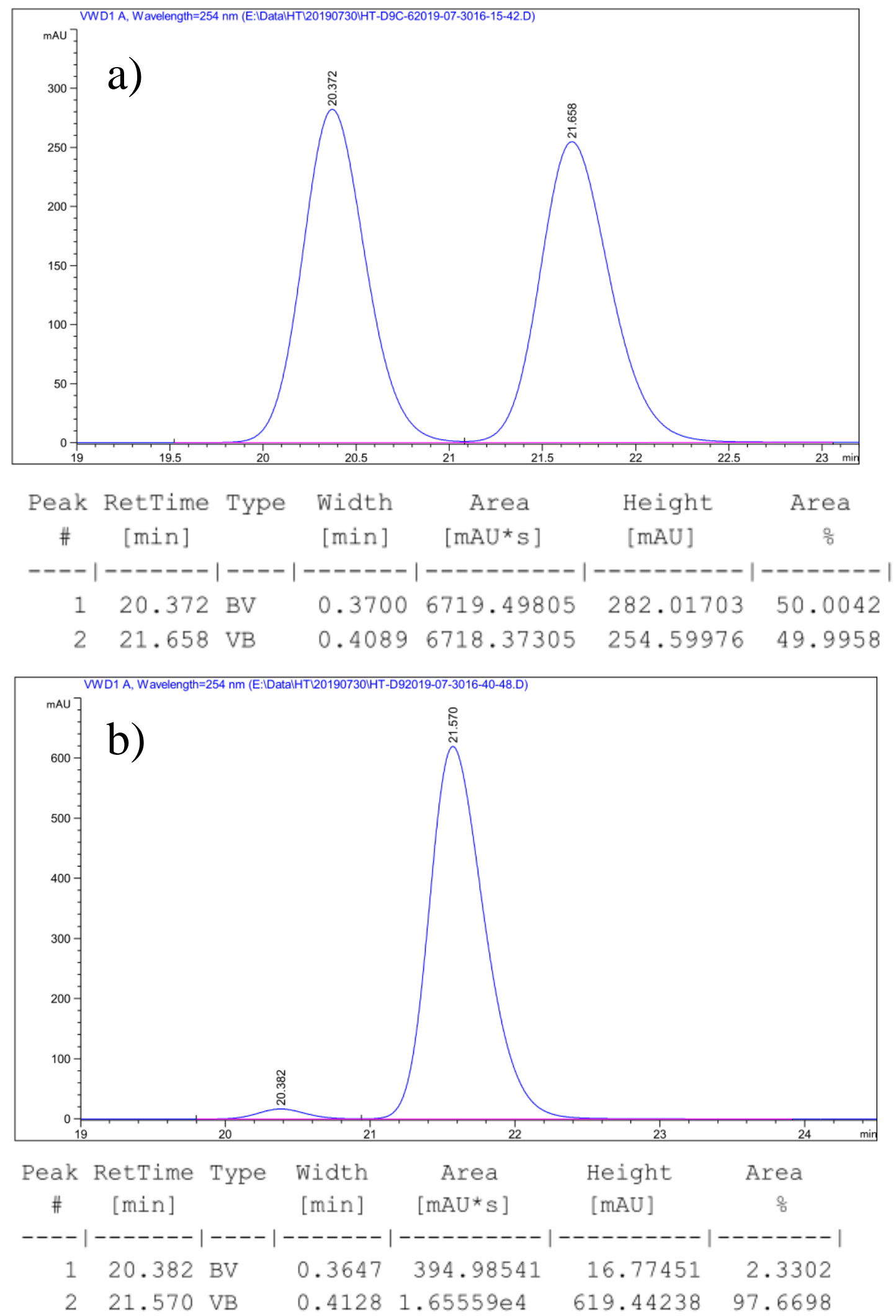

Figure S39. Chiral HPLC analysis of a) racemic 10f and b) (+)-10f (Chiralpak AD-H hexane/2-propanol 99.4/0.6, $1.0 \mathrm{~mL} / \mathrm{min}, \lambda=254 \mathrm{~nm}$ ). 


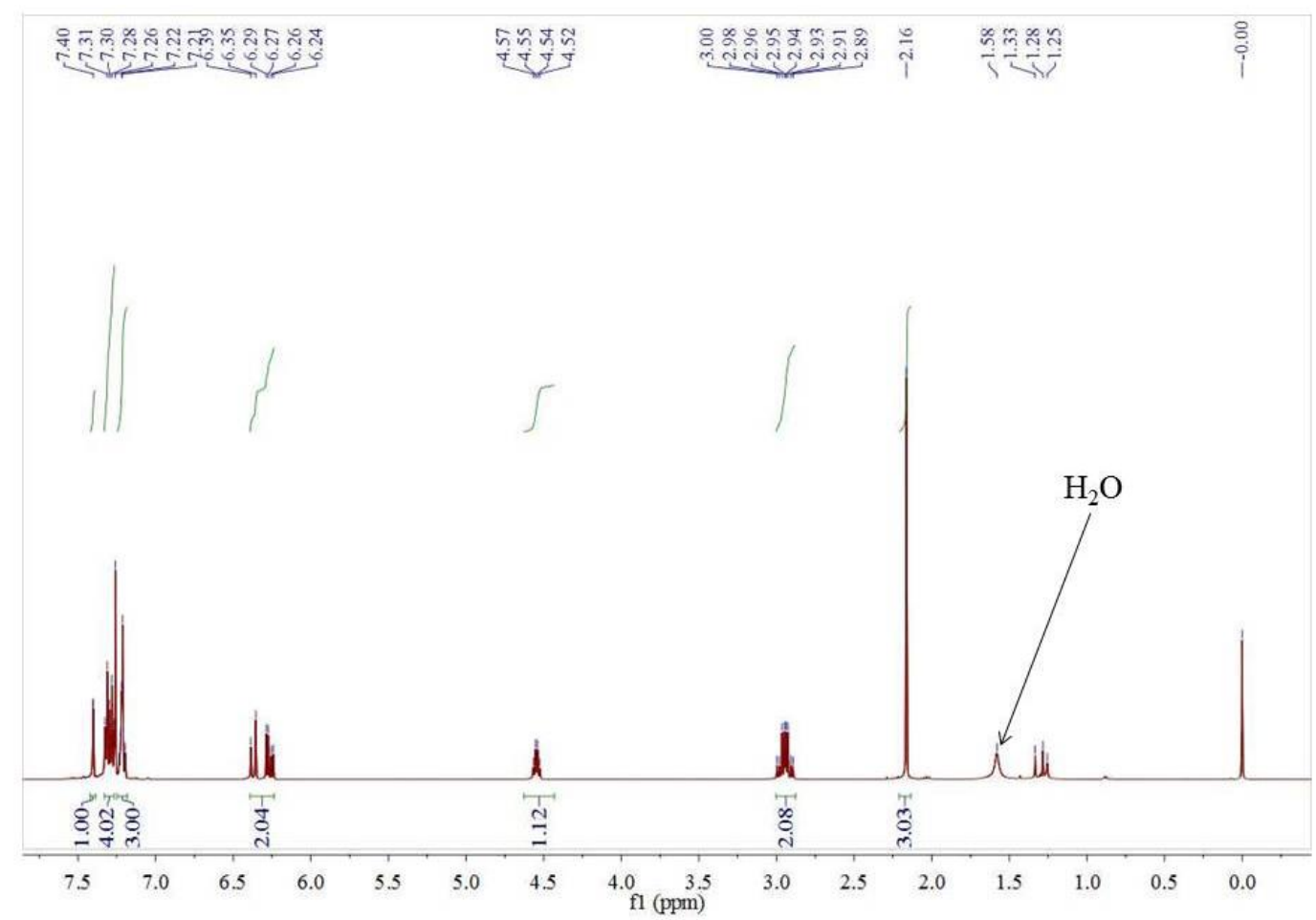

Figure S40. ${ }^{1} \mathrm{H}$ NMR $\left(500 \mathrm{MHz}, \mathrm{CDCl}_{3}, 22{ }^{\circ} \mathrm{C}\right)$ spectrum of $\mathbf{1 0 g}$.

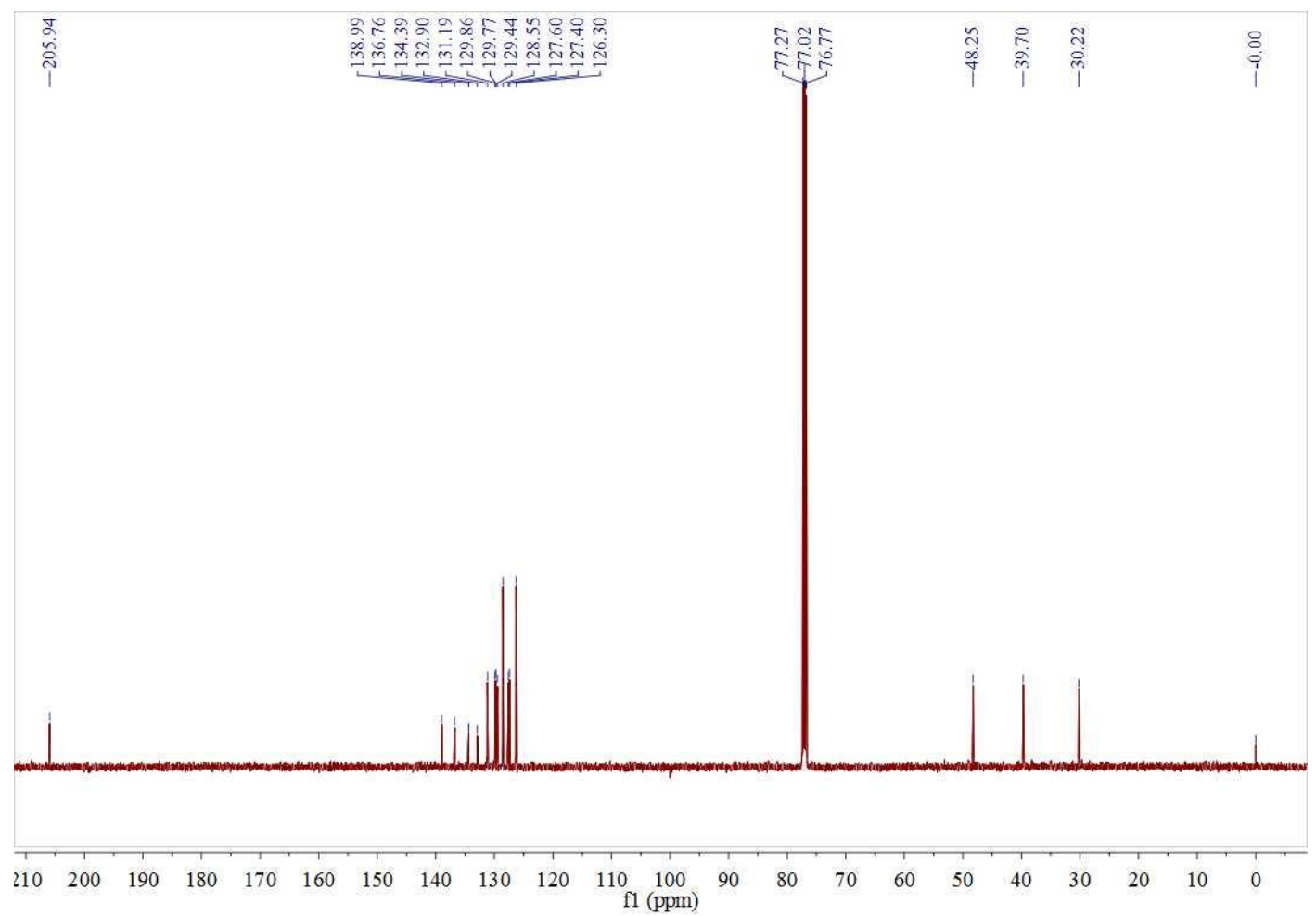

Figure S41. ${ }^{13} \mathrm{C}$ NMR $\left(125 \mathrm{MHz}, \mathrm{CDCl}_{3}, 22{ }^{\circ} \mathrm{C}\right)$ spectrum of $\mathbf{1 0 g}$. 

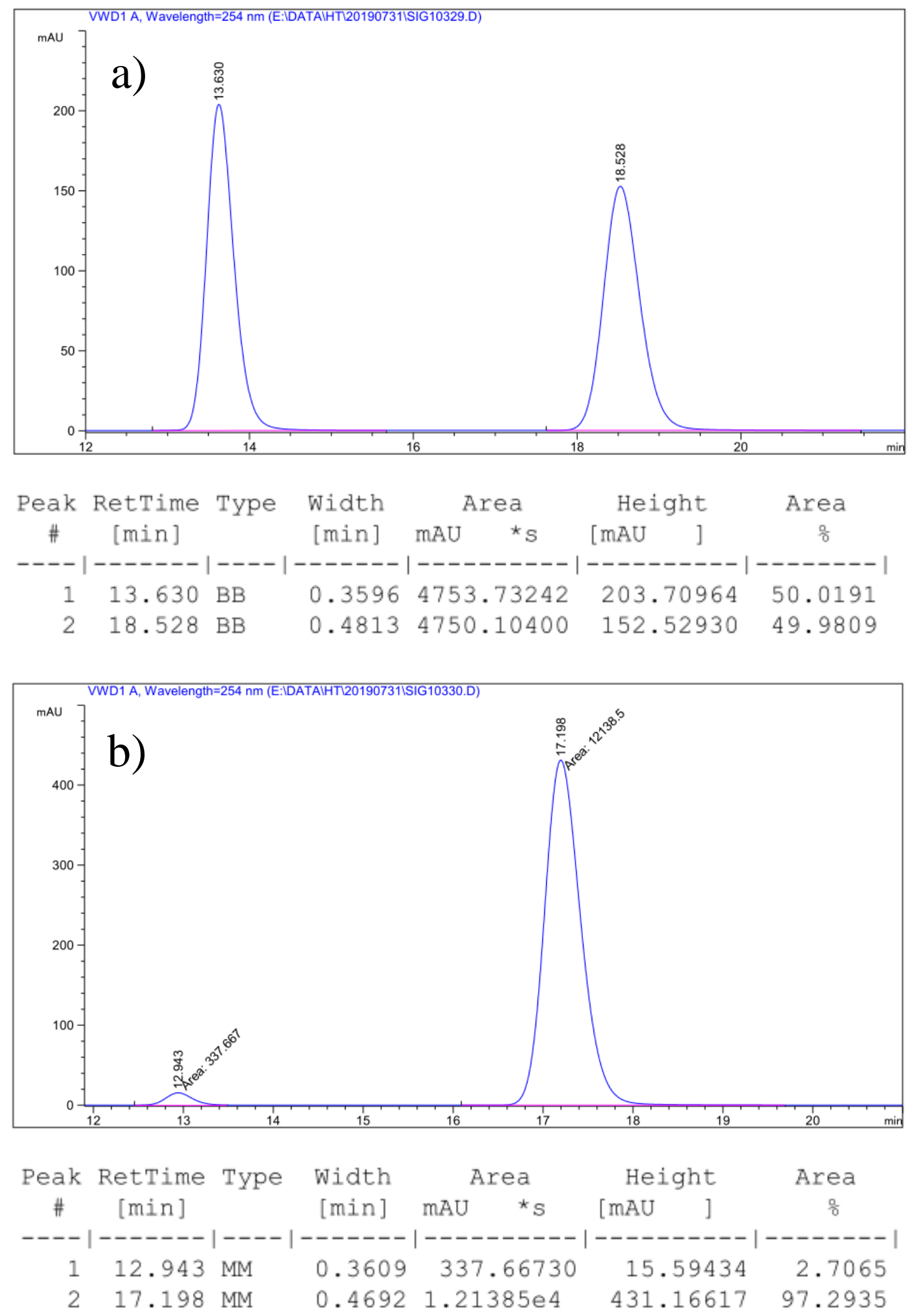

Figure S42. Chiral HPLC analysis of a) racemic $10 \mathrm{~g}$ and b) (-)-10g (Chiralpak OD-H hexane/2-propanol 98.5/1.5, $1.0 \mathrm{~mL} / \mathrm{min}, \lambda=254 \mathrm{~nm}$ ). 


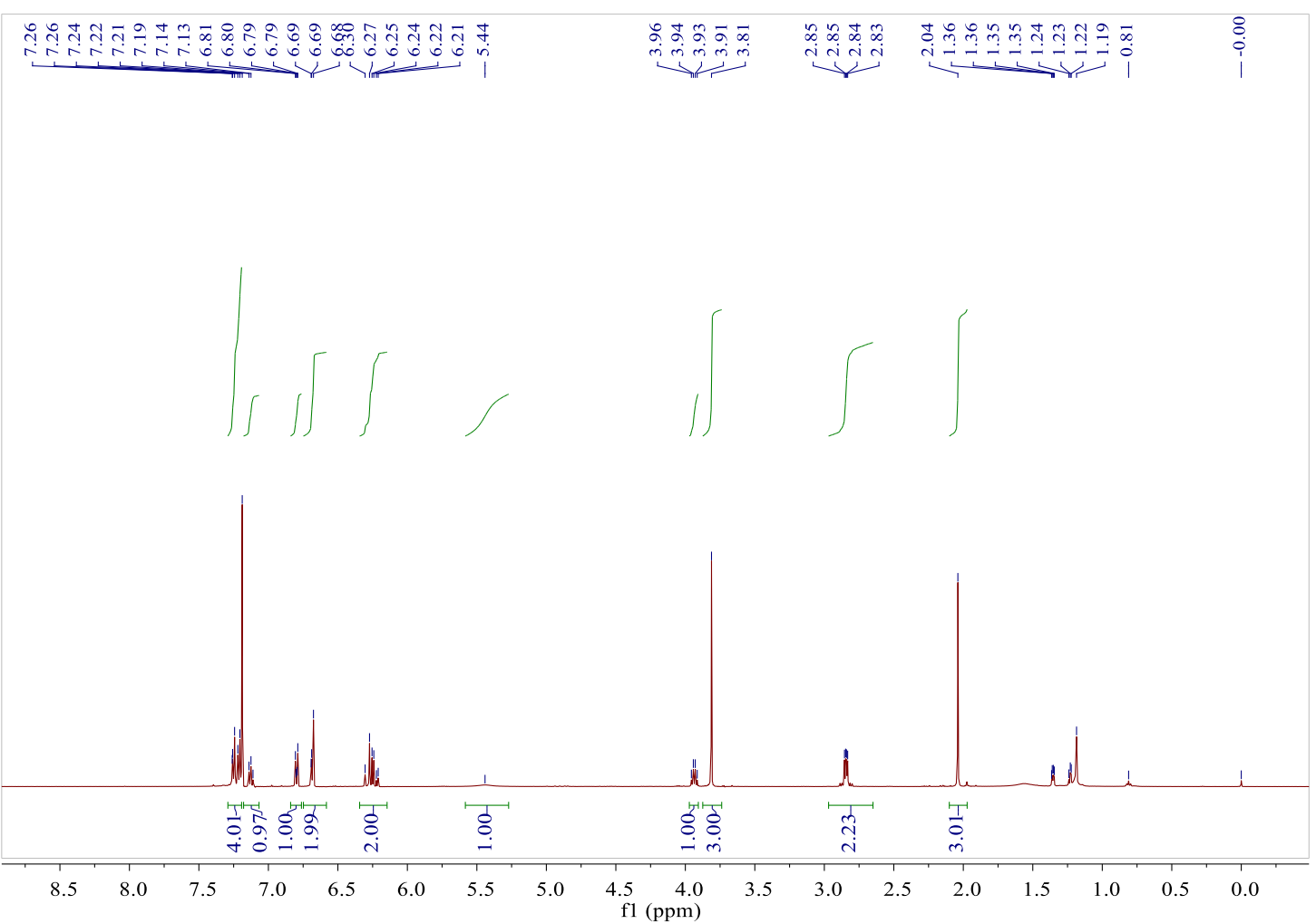

Figure S43. ${ }^{1} \mathrm{H}$ NMR $\left(500 \mathrm{MHz}, \mathrm{CDCl}_{3}, 22{ }^{\circ} \mathrm{C}\right)$ spectrum of $\mathbf{1 0 h}$.

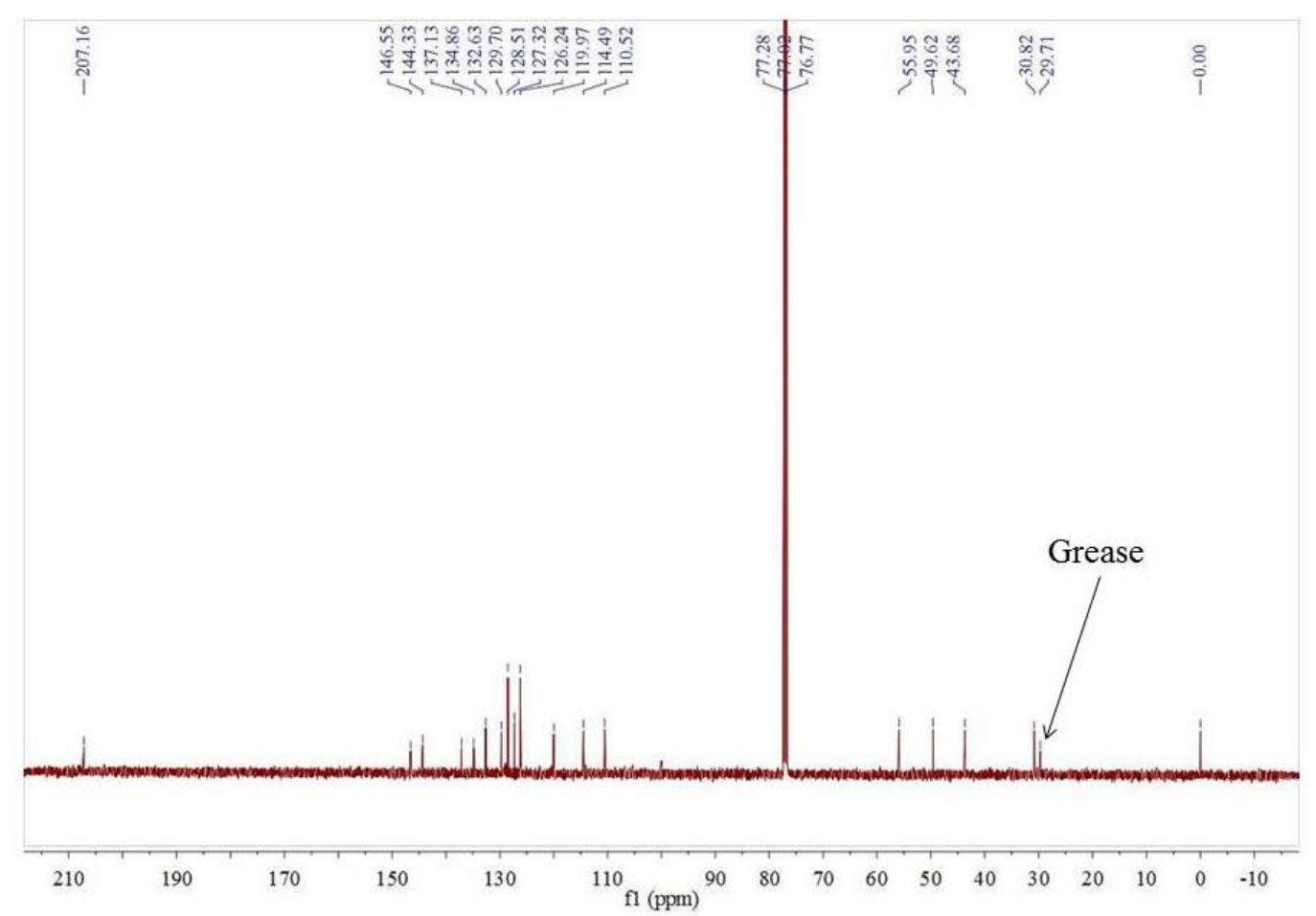

Figure S44. ${ }^{13} \mathrm{C}$ NMR $\left(125 \mathrm{MHz}, \mathrm{CDCl}_{3}, 22{ }^{\circ} \mathrm{C}\right)$ spectrum of $\mathbf{1 0 h}$. 

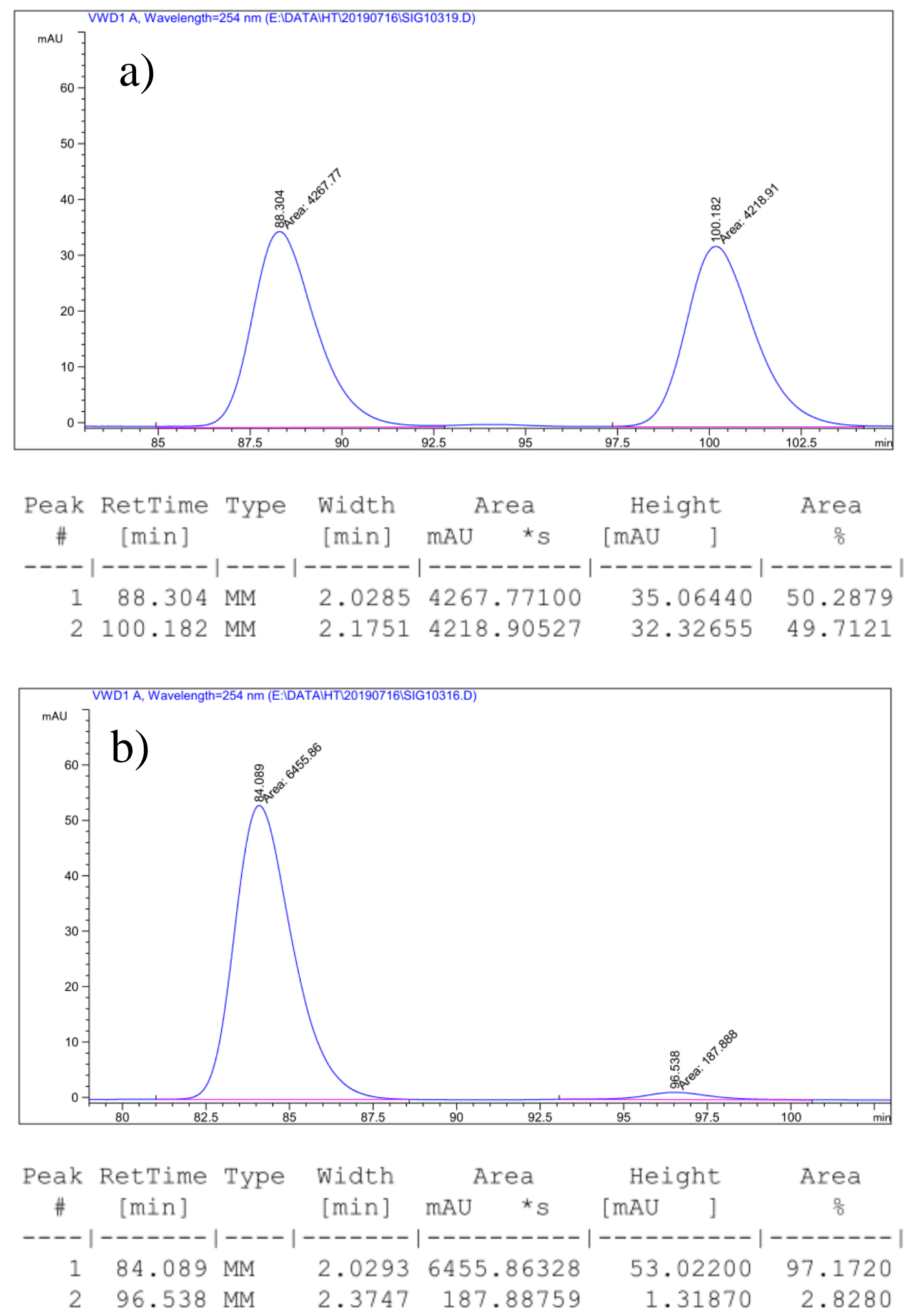

Figure 45. Chiral HPLC analysis of a) racemic 10h and b) (+)-10h (Chiralpak AD-H hexane/2-propanol 98.5/1.5, $1.0 \mathrm{~mL} / \mathrm{min}, \lambda=254 \mathrm{~nm}$ ). 


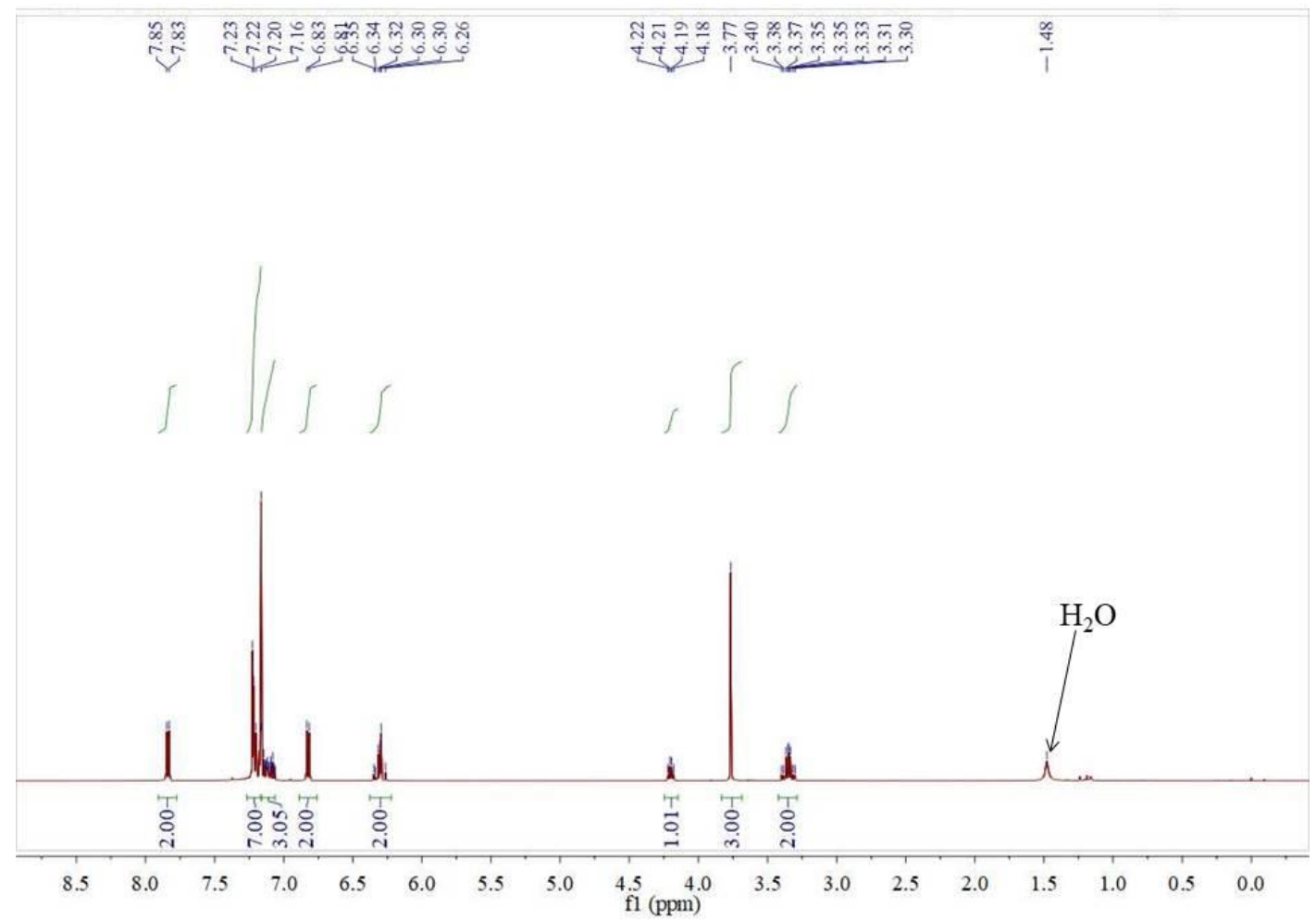

Figure S46. ${ }^{1} \mathrm{H}$ NMR $\left(500 \mathrm{MHz}, \mathrm{CDCl}_{3}, 22^{\circ} \mathrm{C}\right)$ spectrum of $\mathbf{1 0 i}$.

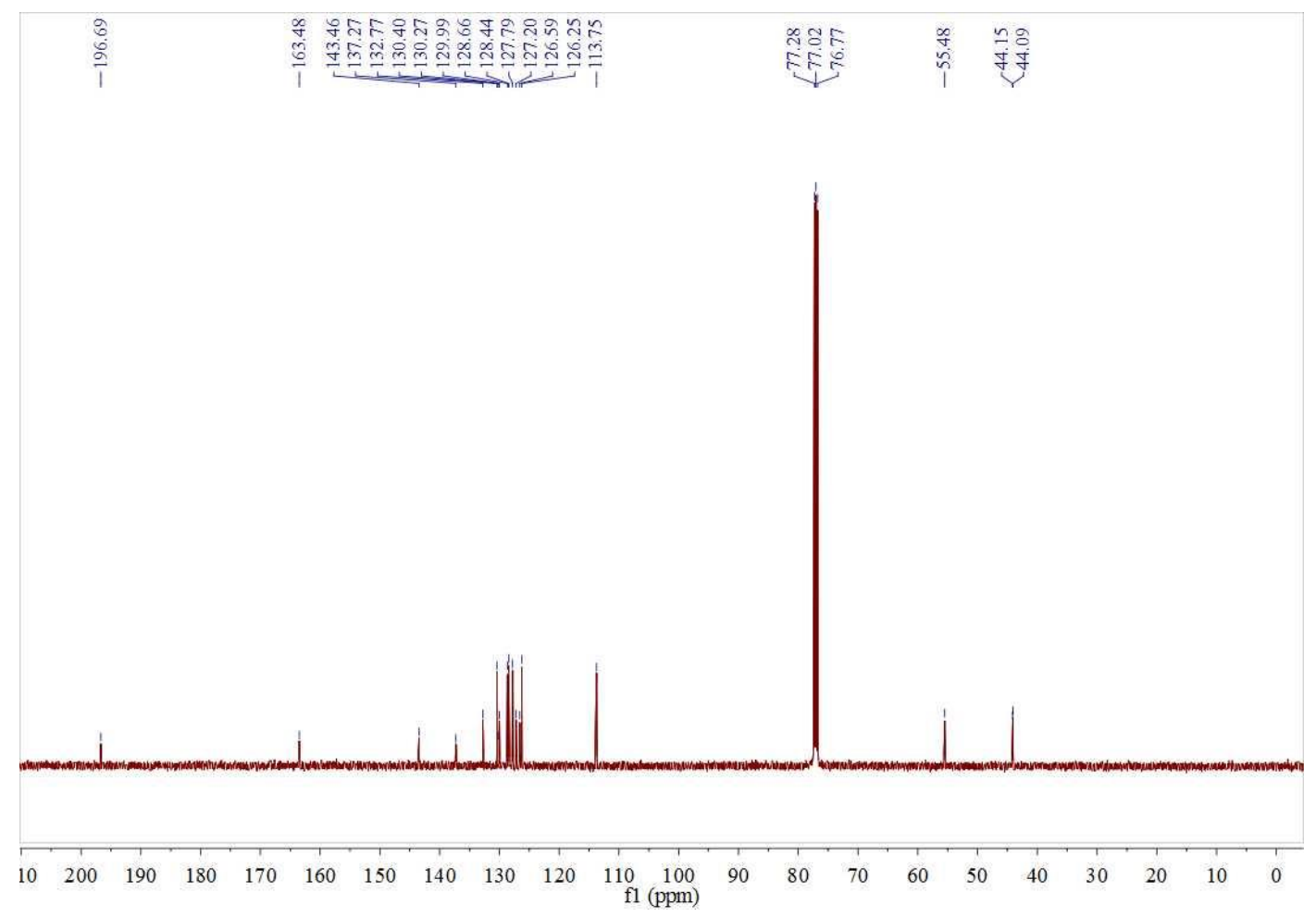

Figure $\mathbf{S 4 7} .{ }^{13} \mathrm{C}$ NMR $\left(125 \mathrm{MHz}, \mathrm{CDCl}_{3}, 22{ }^{\circ} \mathrm{C}\right)$ spectrum of $\mathbf{1 0 i}$. 

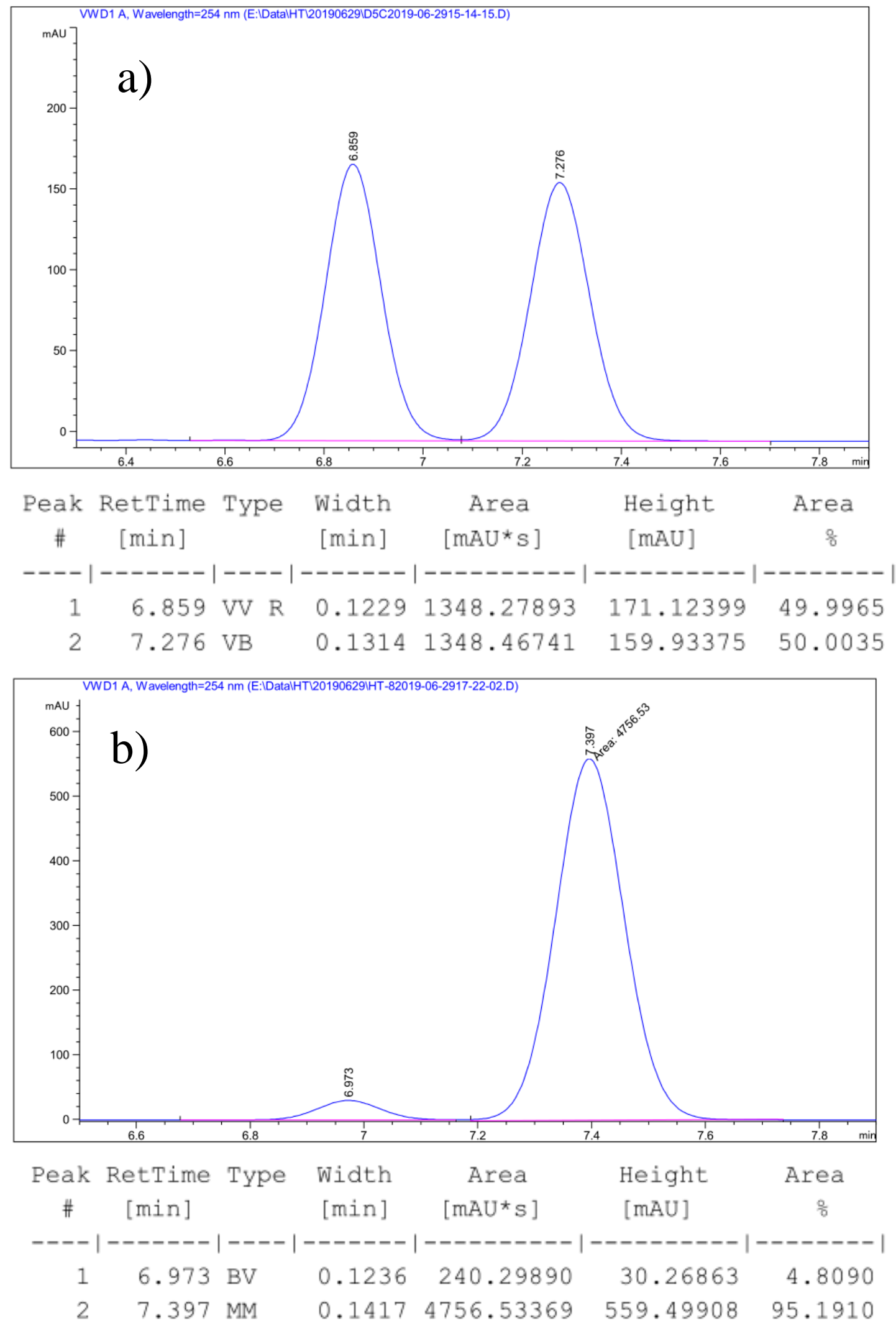

Figure S48. Chiral HPLC analysis of a) racemic 10i and b) (R)-10i (Chiralpak IC, hexane/2-propanol 98.5/1.5, $1.0 \mathrm{~mL} / \mathrm{min}, \lambda=254 \mathrm{~nm}$ ). 


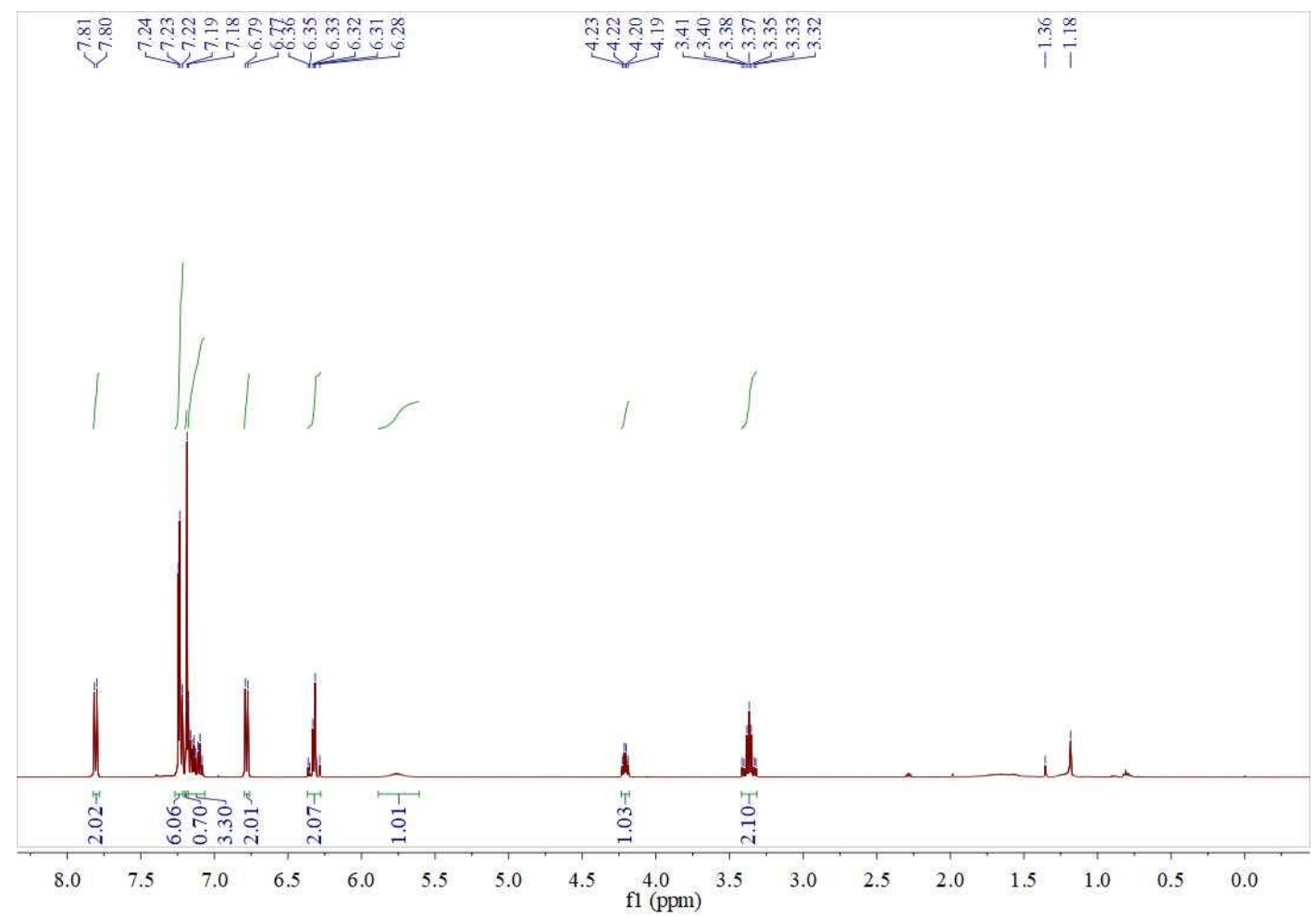

Figure S49. ${ }^{1} \mathrm{H} \mathrm{NMR}\left(500 \mathrm{MHz}, \mathrm{CDCl}_{3}, 22{ }^{\circ} \mathrm{C}\right)$ spectrum of $\mathbf{1 0 j}$.

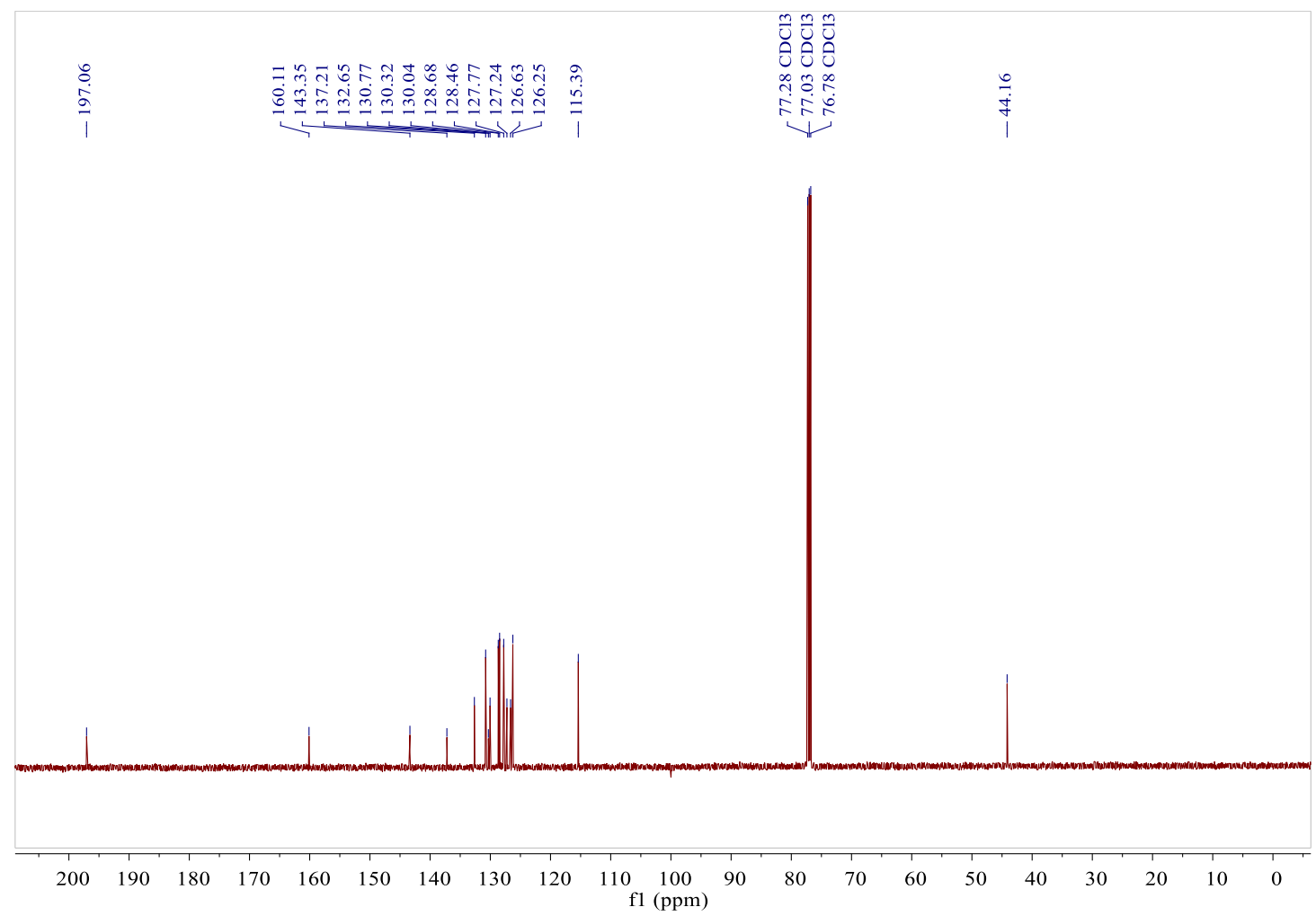

Figure S50. ${ }^{13} \mathrm{C}$ NMR $\left(125 \mathrm{MHz}, \mathrm{CDCl}_{3}, 22{ }^{\circ} \mathrm{C}\right)$ spectrum of $\mathbf{1 0 j}$. 

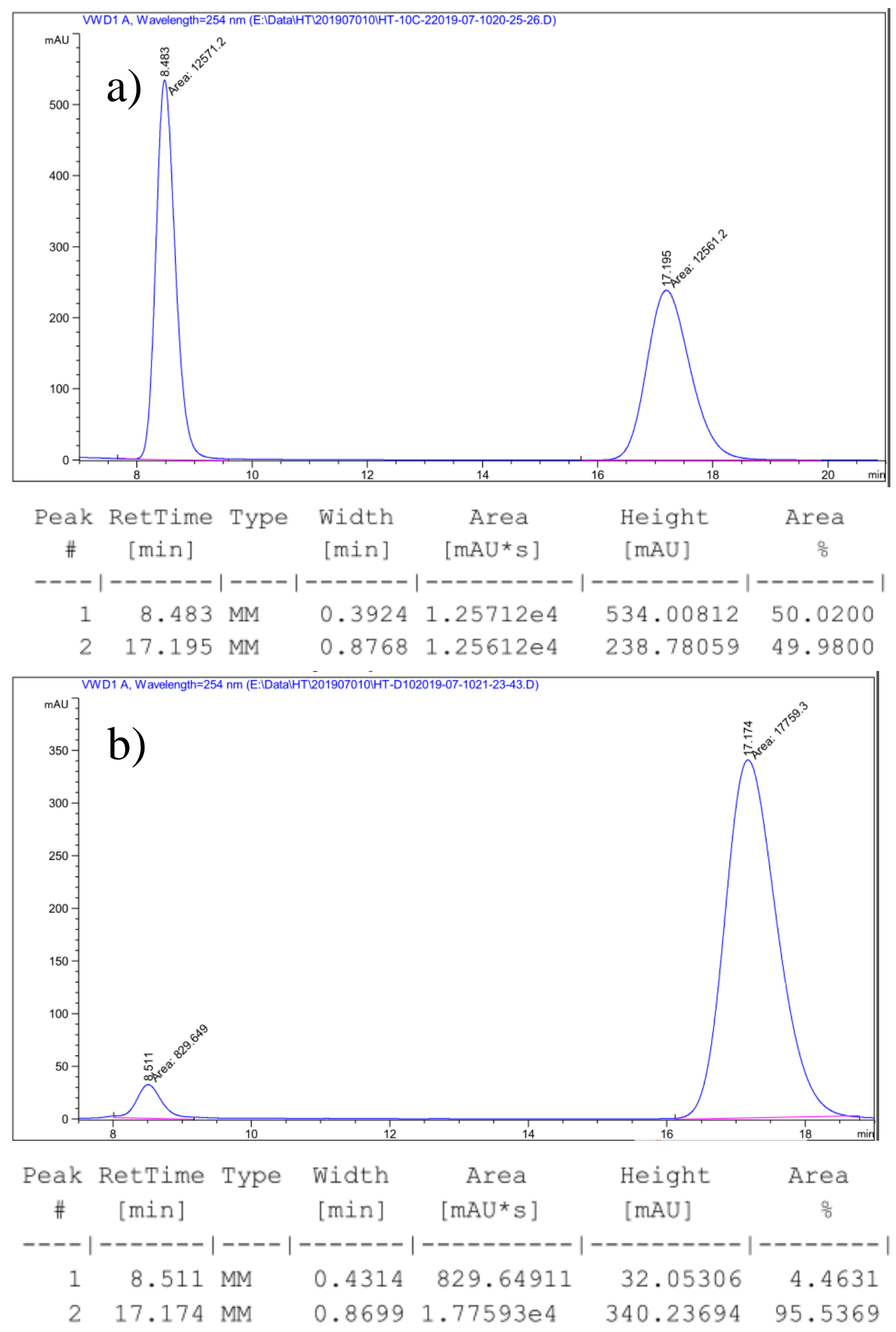

Figure S51. Chiral HPLC analysis of a) racemic 10j and b) (+)-10j (Chiralpak OD-H hexane/2-propanol 90/10, $1.0 \mathrm{~mL} / \mathrm{min}, \lambda=245 \mathrm{~nm}$ ). 


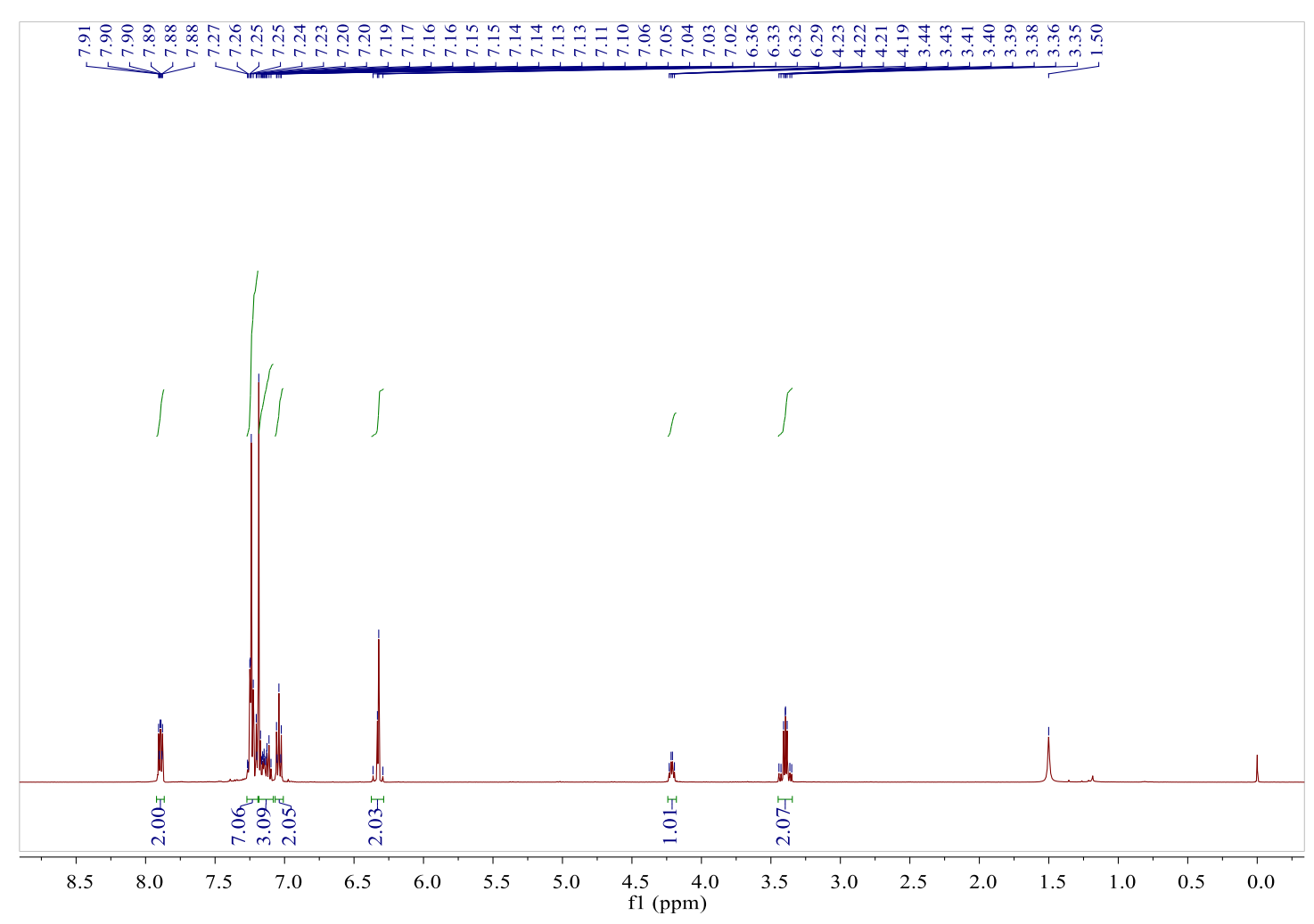

Figure S52. ${ }^{1} \mathrm{H}$ NMR $\left(500 \mathrm{MHz}, \mathrm{CDCl}_{3}, 22{ }^{\circ} \mathrm{C}\right)$ spectrum of $\mathbf{1 0 k}$.

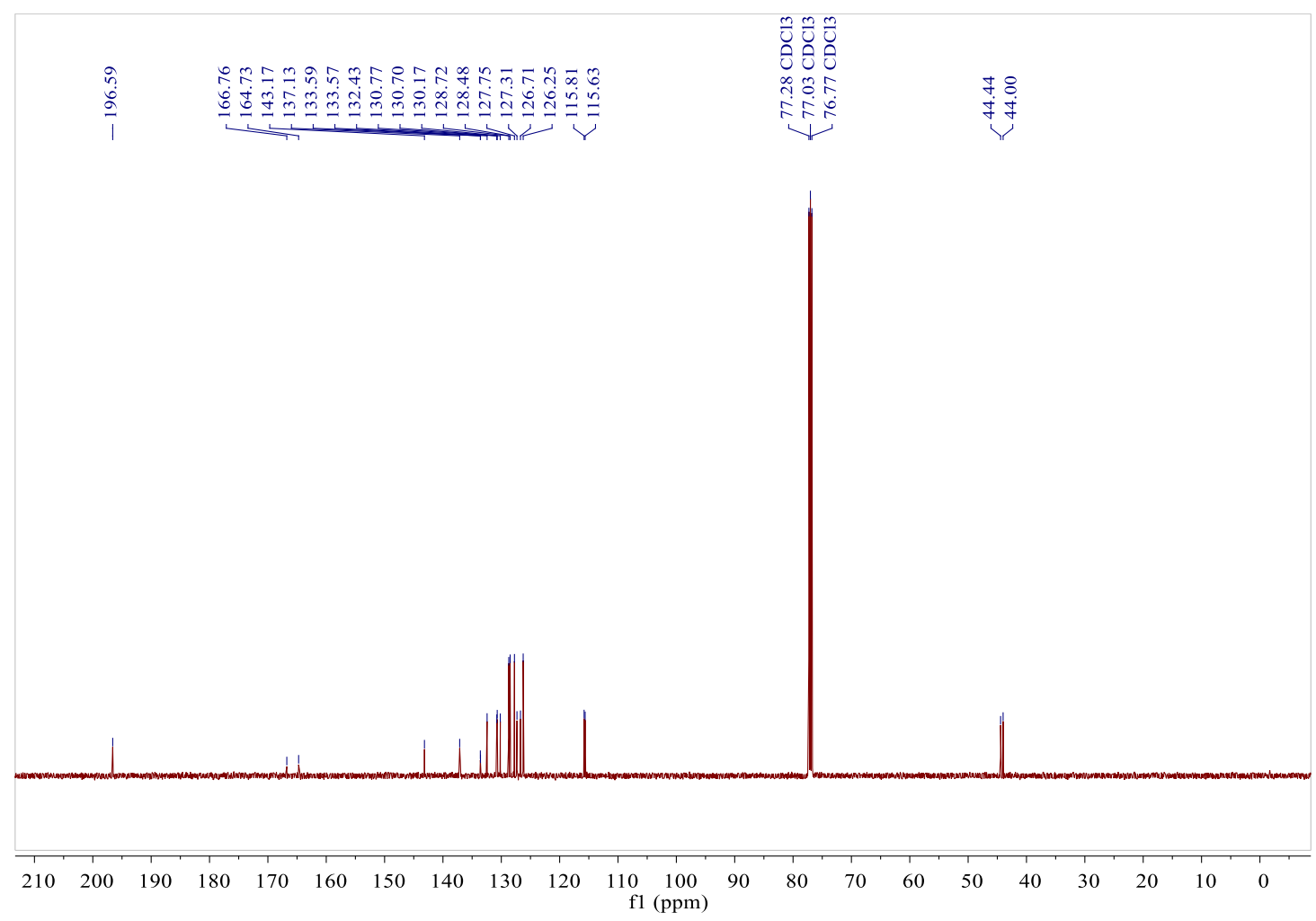

Figure S53. ${ }^{13} \mathrm{C}$ NMR $\left(125 \mathrm{MHz}, \mathrm{CDCl}_{3}, 22{ }^{\circ} \mathrm{C}\right)$ spectrum of $\mathbf{1 0 k}$. 

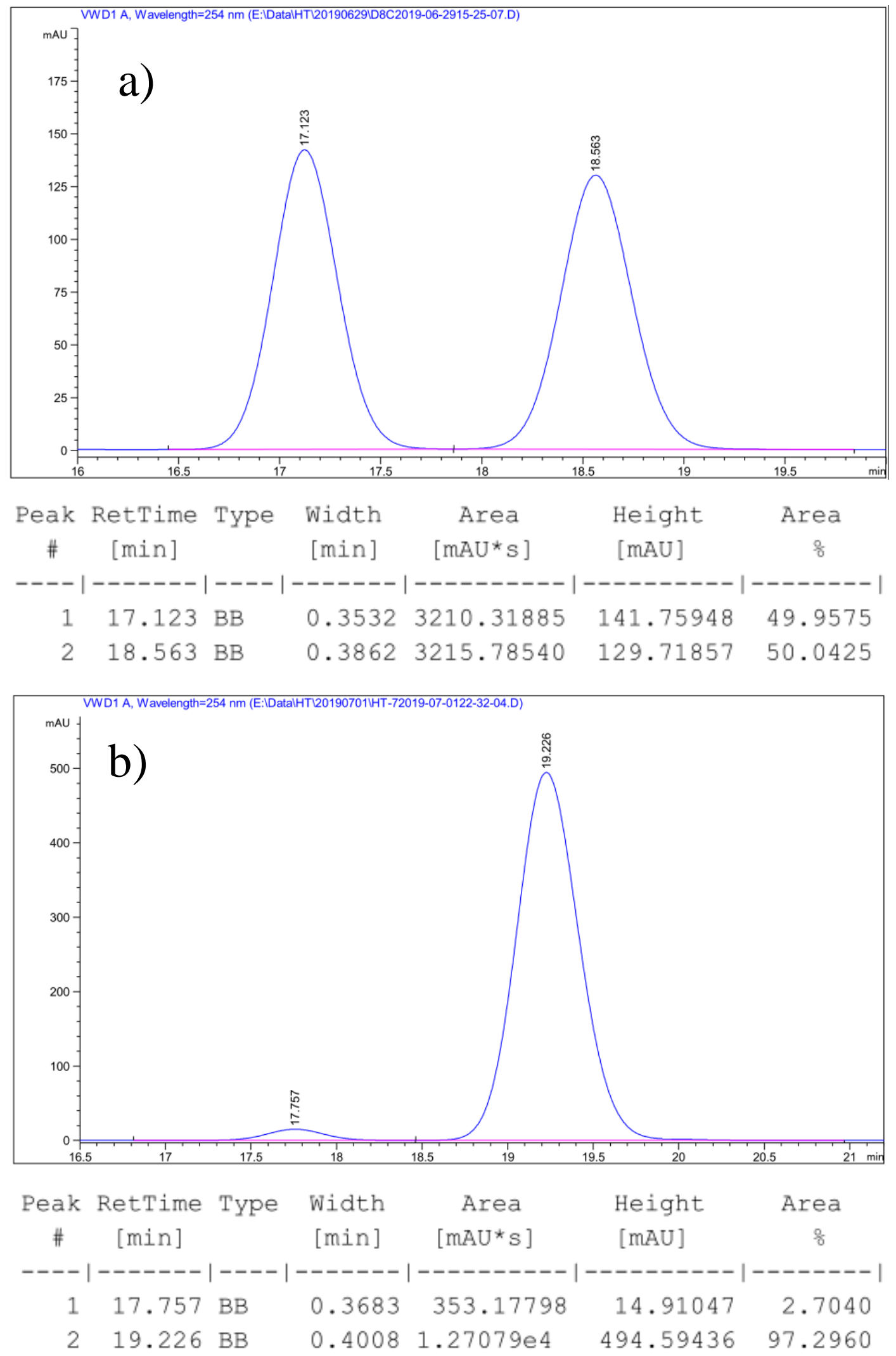

Figure S54. Chiral HPLC analysis of a) racemic 10k and b) (+)-10k. (Chiralpak IC, hexane/2-propanol 98.5/1.5, $1.0 \mathrm{~mL} / \mathrm{min}, \lambda=254 \mathrm{~nm}$ ). 


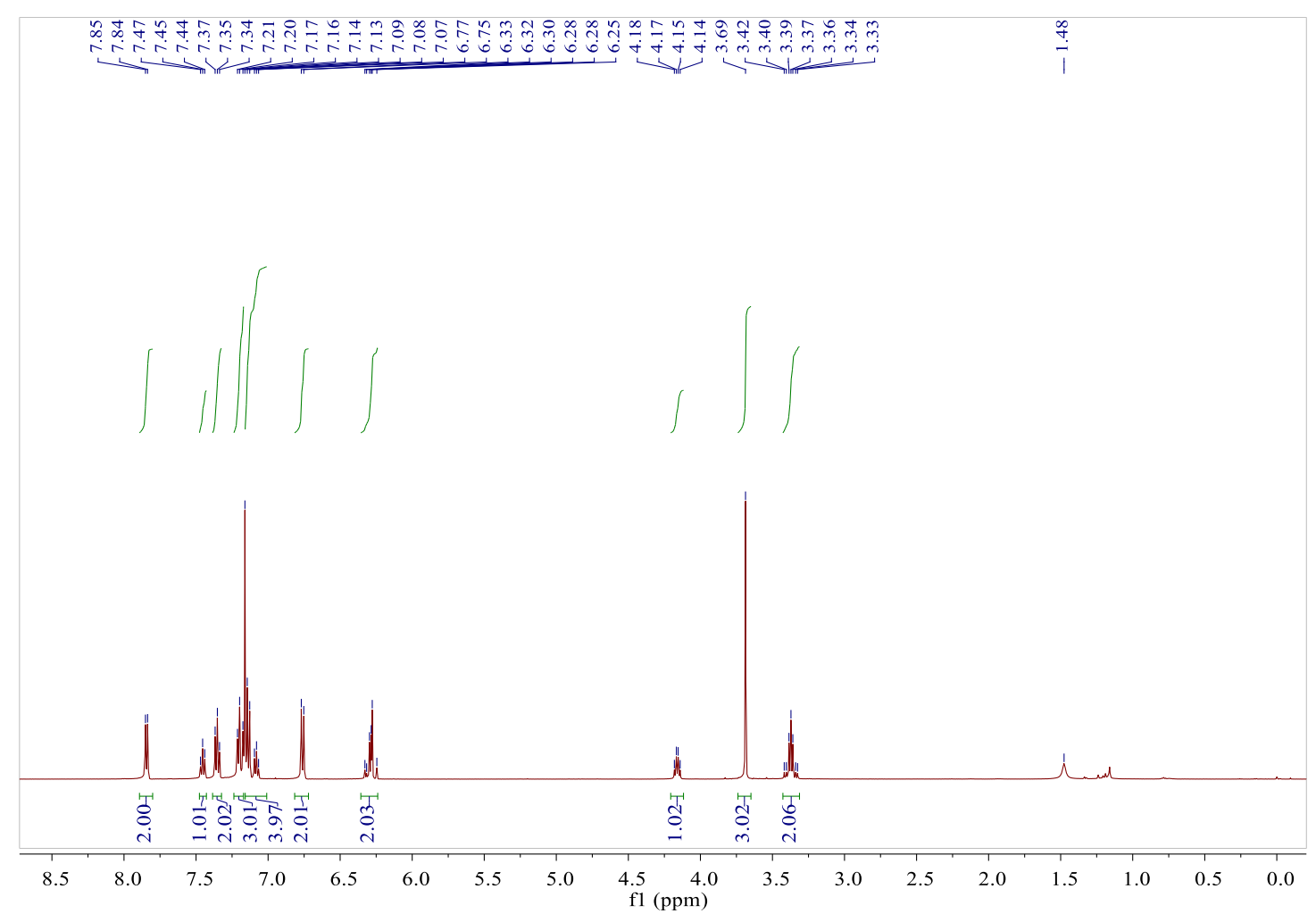

Figure S55. ${ }^{1} \mathrm{H}$ NMR $\left(500 \mathrm{MHz}, \mathrm{CDCl}_{3}, 22{ }^{\circ} \mathrm{C}\right)$ spectrum of $\mathbf{1 0 1}$.

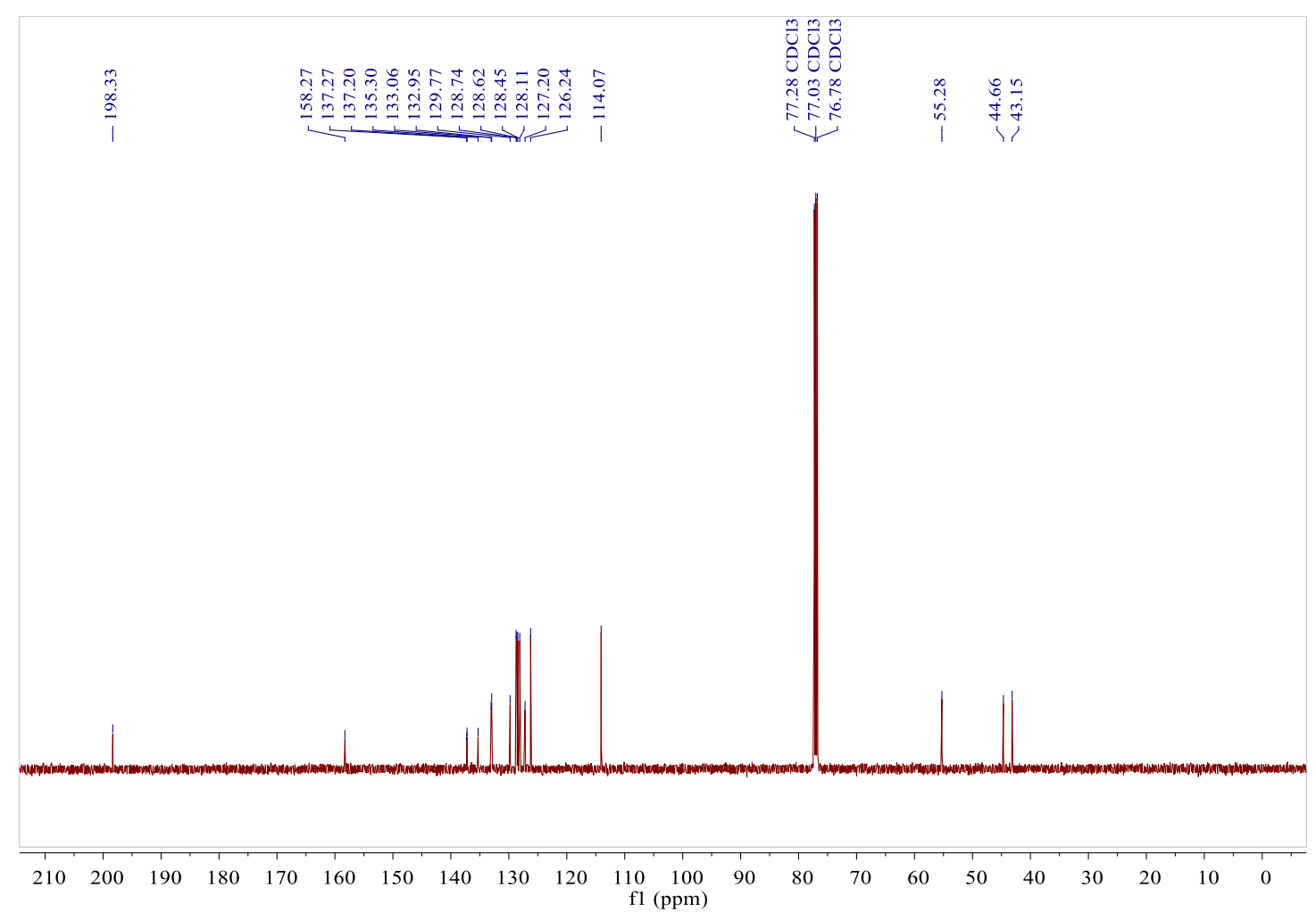

Figure S56. ${ }^{13} \mathrm{C}$ NMR $\left(125 \mathrm{MHz}, \mathrm{CDCl}_{3}, 22{ }^{\circ} \mathrm{C}\right)$ spectrum of $\mathbf{1 0 1}$. 

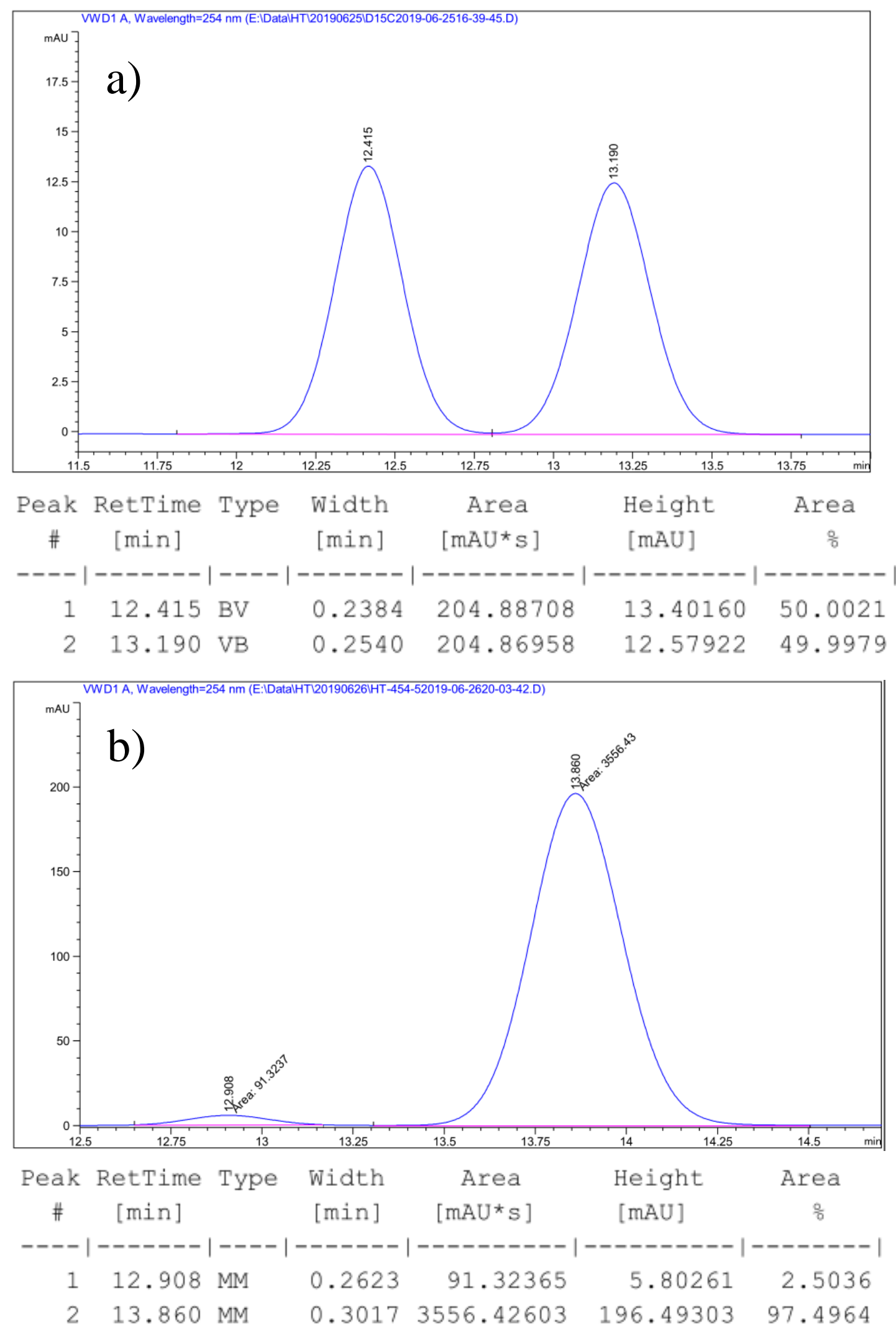

Figure S57. Chiral HPLC analysis of a) racemic 101 and b) $(R)-101$ (Chiralpak IC, hexane/2-propanol 98.5/1.5, $1.0 \mathrm{~mL} / \mathrm{min}, \lambda=254 \mathrm{~nm}$ ). 


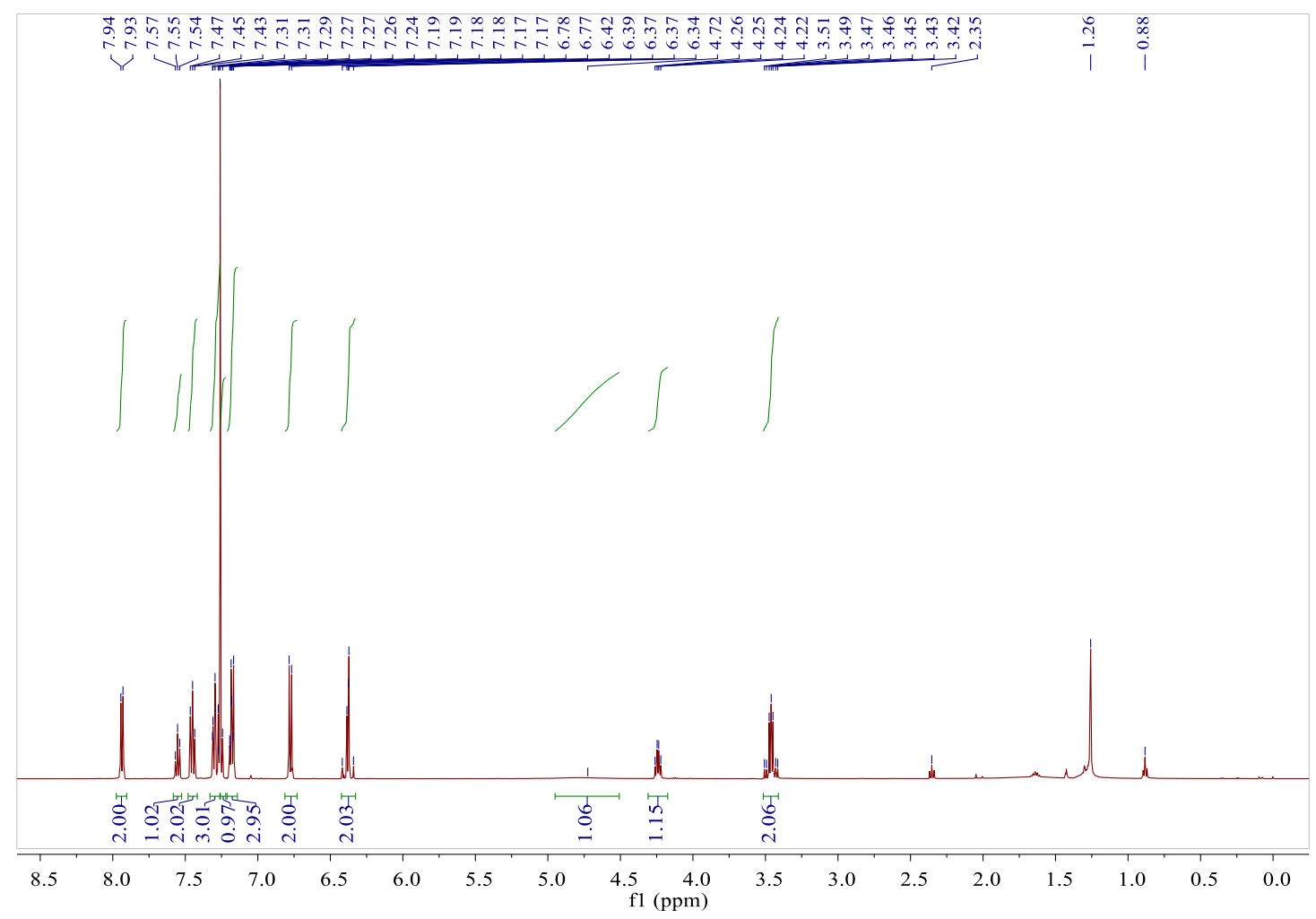

Figure S58. ${ }^{1} \mathrm{H}$ NMR $\left(500 \mathrm{MHz}, \mathrm{CDCl}_{3}, 22{ }^{\circ} \mathrm{C}\right)$ spectrum of $\mathbf{1 0 m}$.

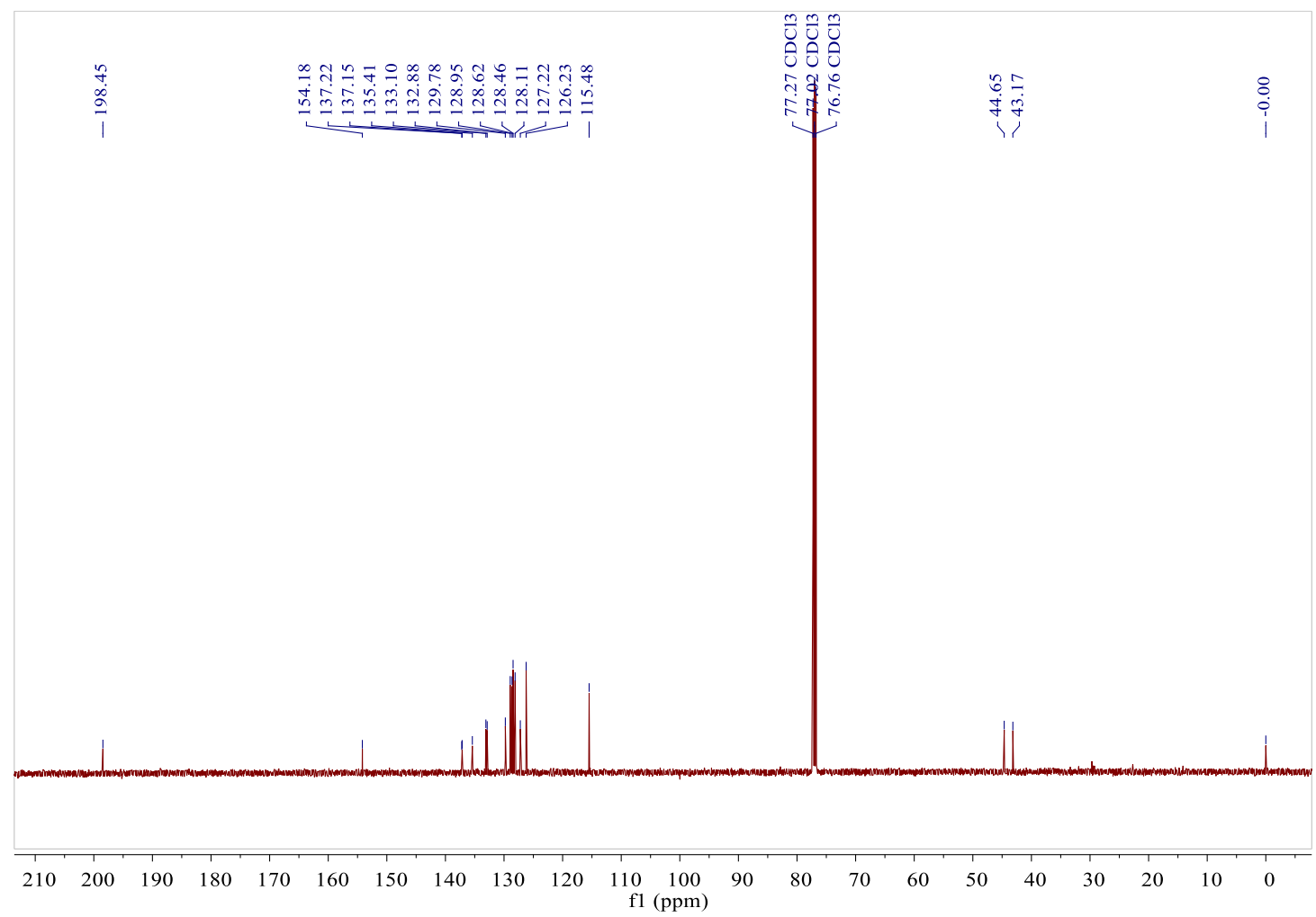

Figure S59. ${ }^{13} \mathrm{C}$ NMR $\left(125 \mathrm{MHz}, \mathrm{CDCl}_{3}, 22{ }^{\circ} \mathrm{C}\right)$ spectrum of $\mathbf{1 0 m}$. 

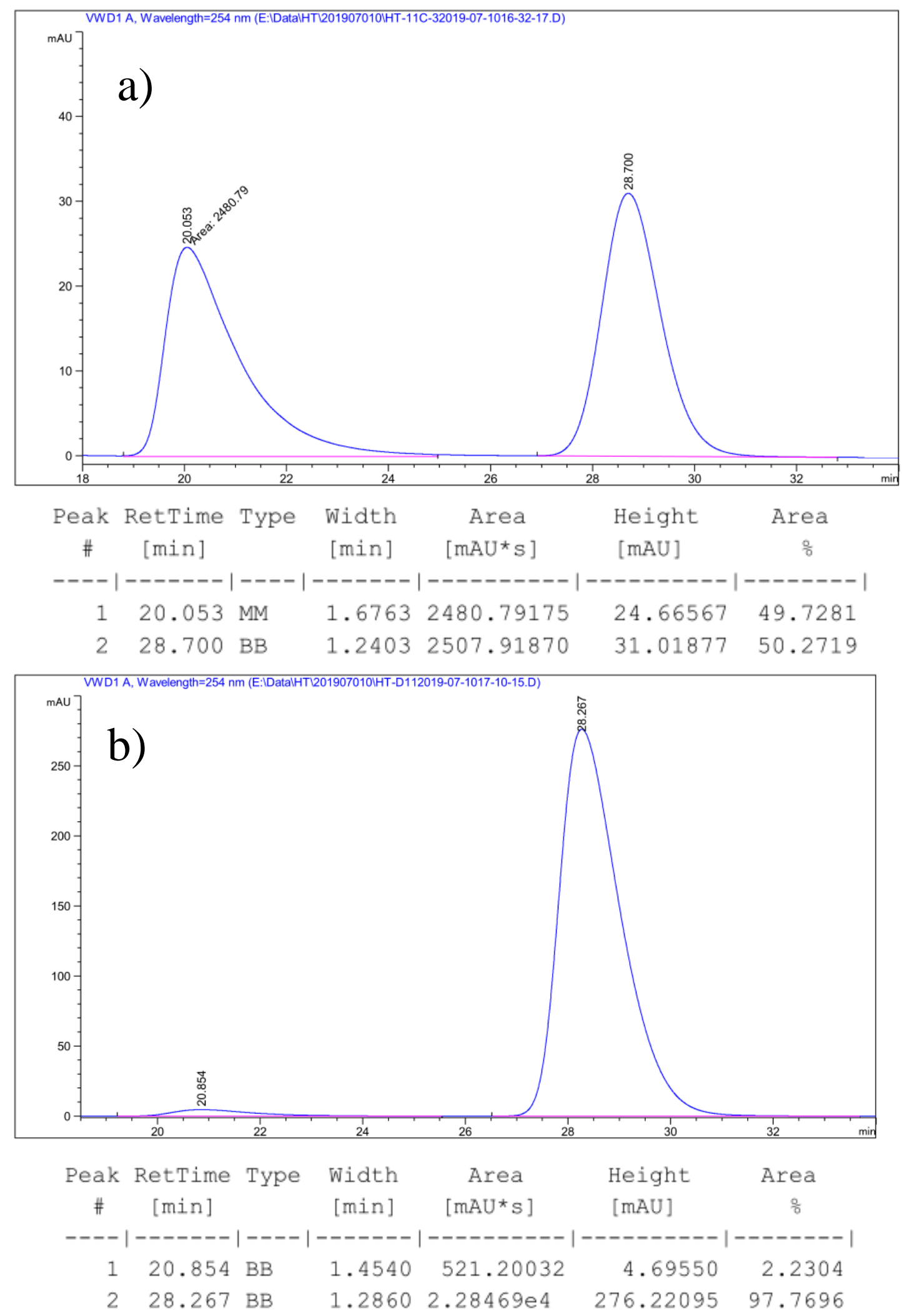

Figure S60. Chiral HPLC analysis of a) racemic 10m and b) (+)-10m (Chiralpak OD-H hexane/2-propanol 90/10, $1.0 \mathrm{~mL} / \mathrm{min}, \lambda=254 \mathrm{~nm}$ ). 


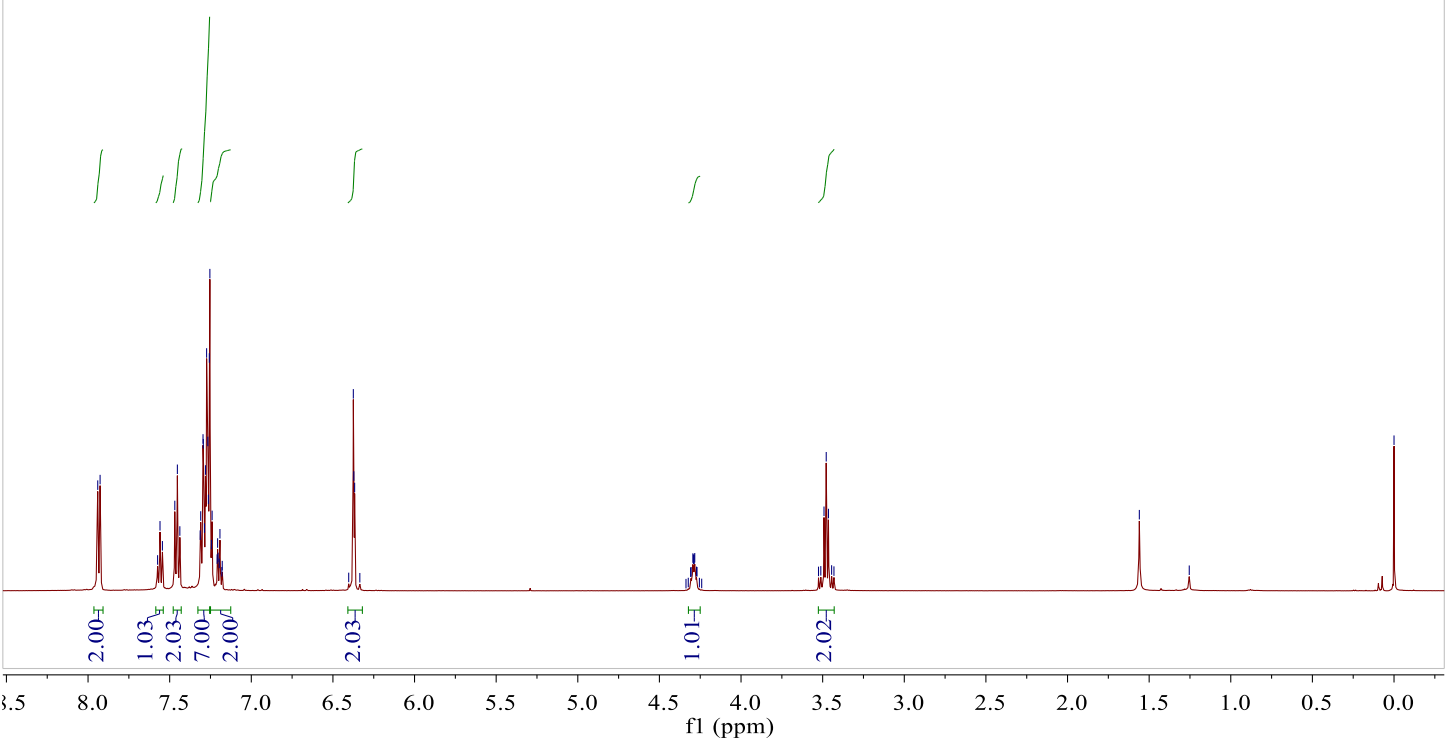

Figure S61. ${ }^{1} \mathrm{H}$ NMR $\left(500 \mathrm{MHz}, \mathrm{CDCl}_{3}, 22{ }^{\circ} \mathrm{C}\right)$ spectrum of $\mathbf{1 0 n}$.

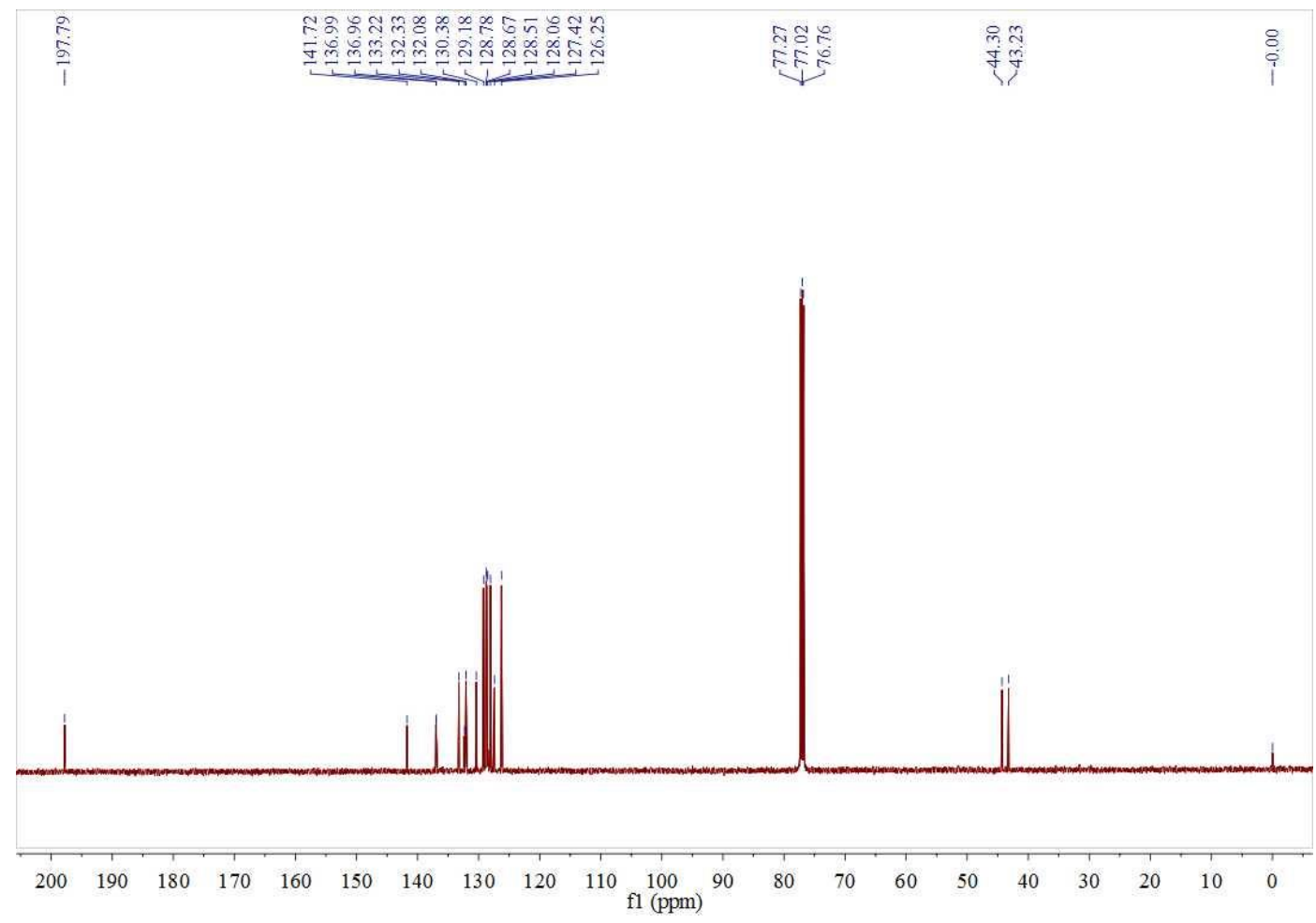

Figure S62. ${ }^{13} \mathrm{C}$ NMR $\left(125 \mathrm{MHz}, \mathrm{CDCl}_{3}, 22{ }^{\circ} \mathrm{C}\right)$ spectrum of $\mathbf{1 0 n}$. 

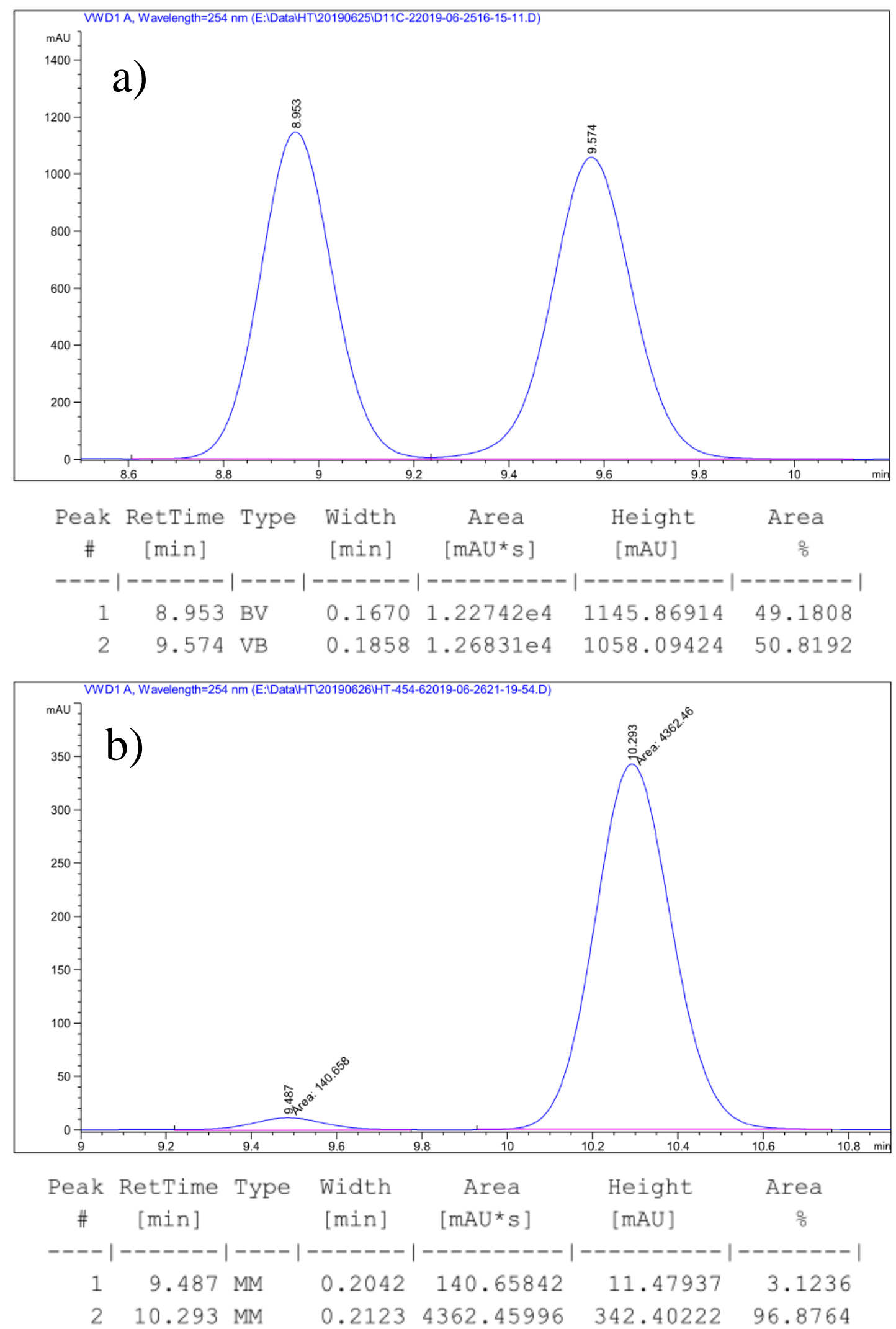

Figure S63. Chiral HPLC analysis of a) racemic 10n and b) (R)-10n (Chiralpak IC hexane/2-propanol 98.5/1.5, $1.0 \mathrm{~mL} / \mathrm{min}, \lambda=254 \mathrm{~nm})$. 


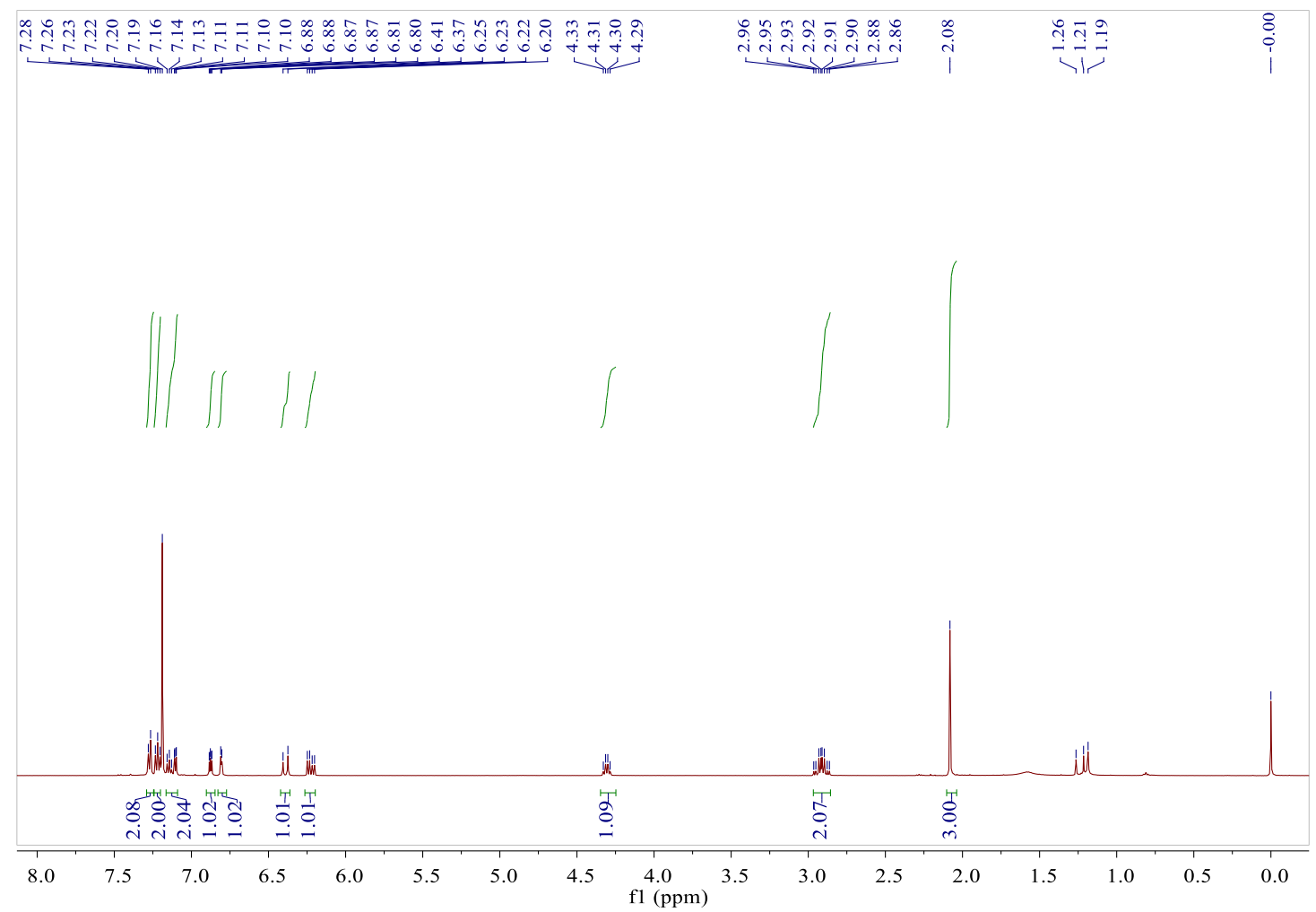

Figure S64. ${ }^{1} \mathrm{H}$ NMR $\left(500 \mathrm{MHz}, \mathrm{CDCl}_{3}, 22{ }^{\circ} \mathrm{C}\right)$ spectrum of $\mathbf{1 0 o}$.

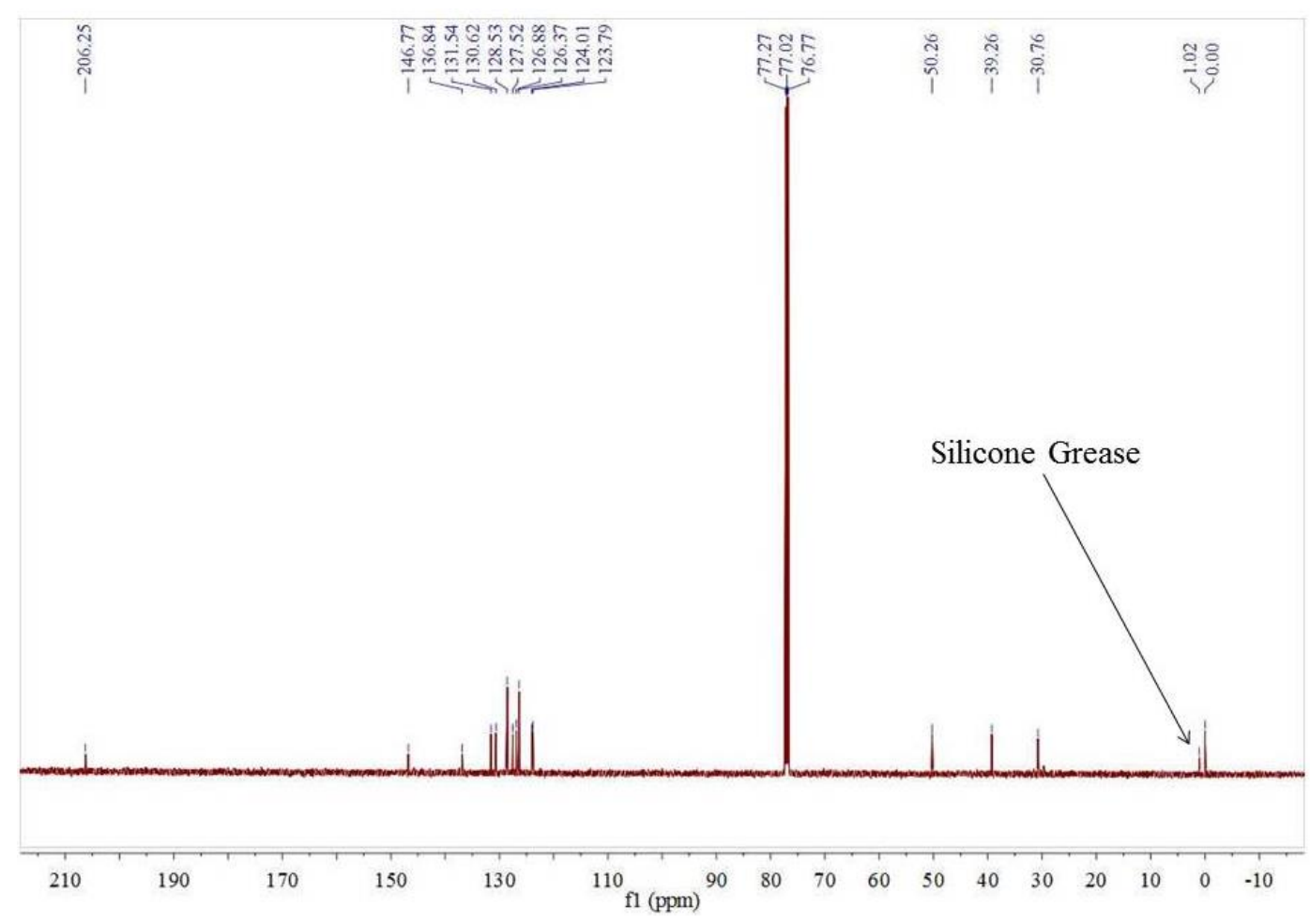

Figure S65. ${ }^{13} \mathrm{C}$ NMR $\left(125 \mathrm{MHz}, \mathrm{CDCl}_{3}, 22{ }^{\circ} \mathrm{C}\right)$ spectrum of $\mathbf{1 0 o}$. 

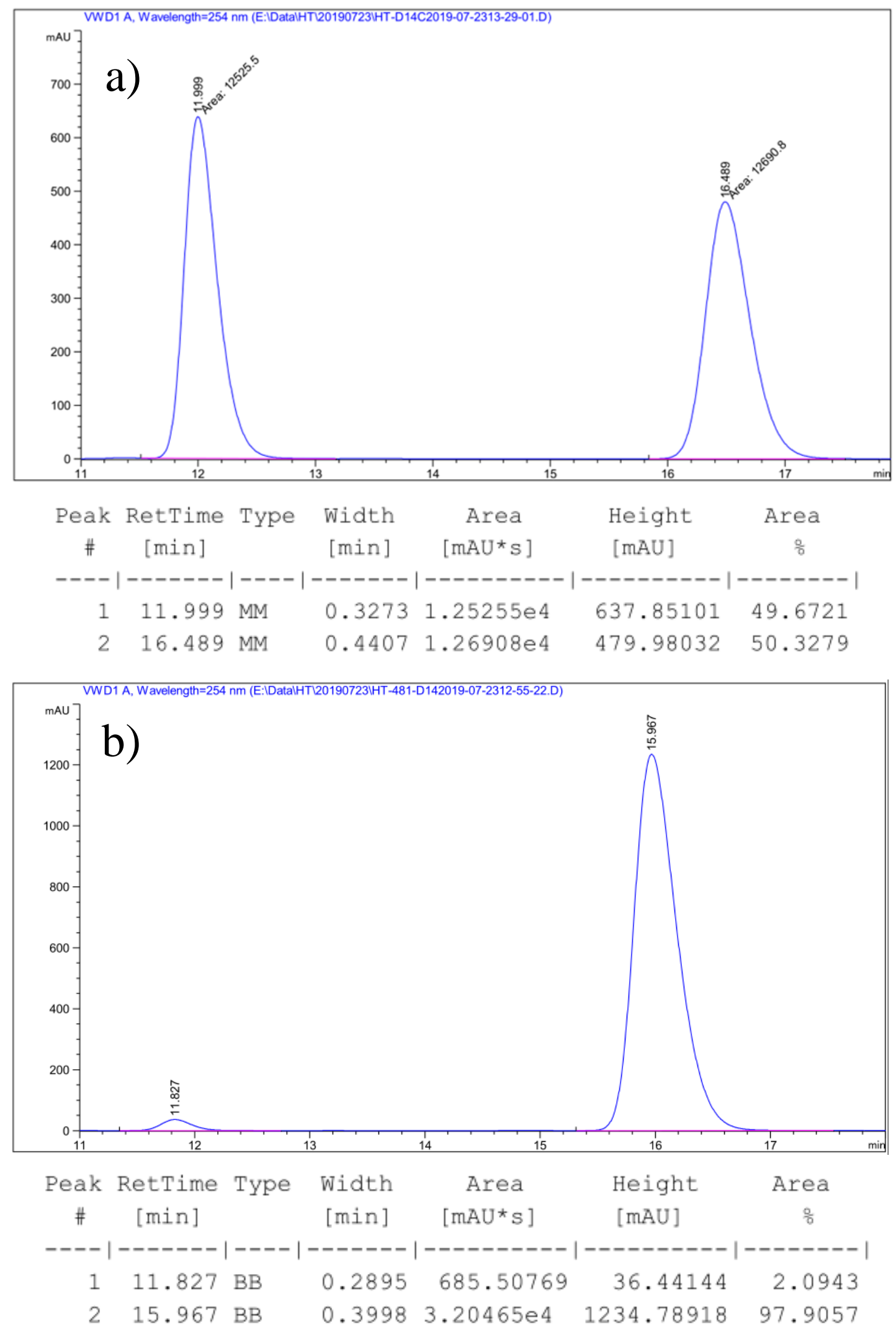

Figure S66. Chiral HPLC analysis of a) racemic 10o and b) (+)-10o (Chiralpak OD-H hexane/2-propanol 98.5/1.5, $1.0 \mathrm{~mL} / \mathrm{min}, \lambda=254 \mathrm{~nm})$. 


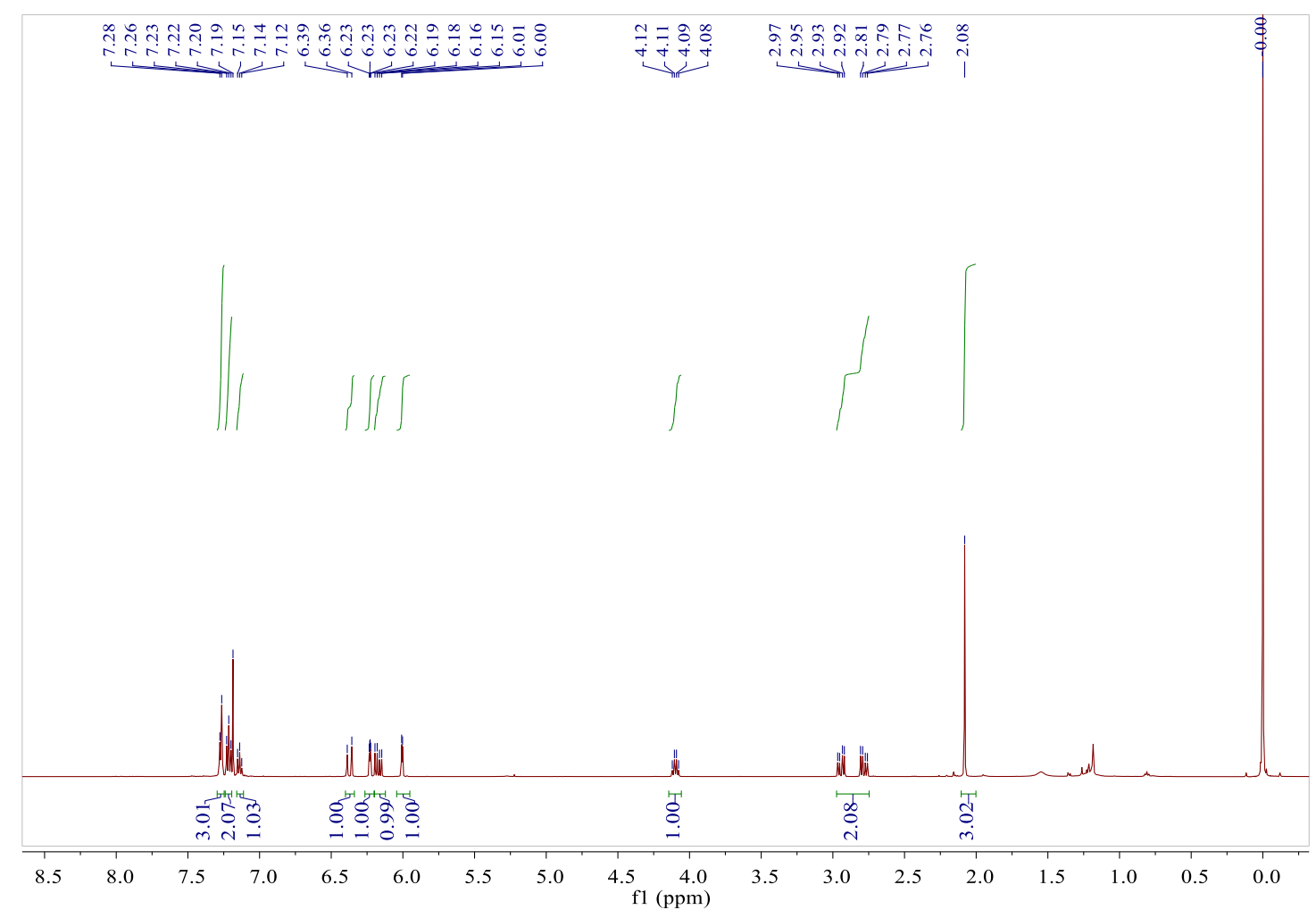

Figure S67. ${ }^{1} \mathrm{H} \mathrm{NMR}\left(500 \mathrm{MHz}, \mathrm{CDCl}_{3}, 22{ }^{\circ} \mathrm{C}\right)$ spectrum of $\mathbf{1 0 p}$.

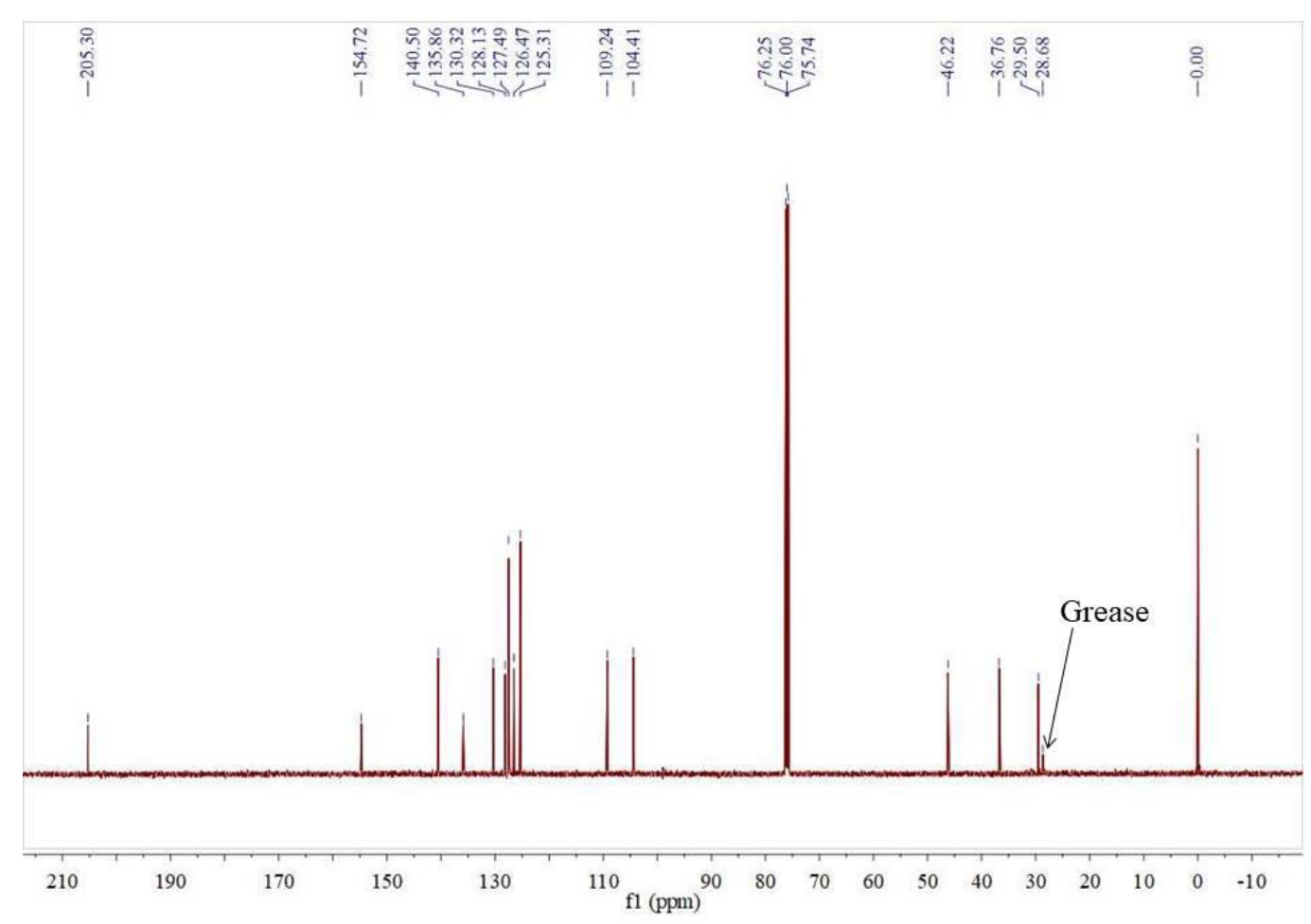

Figure S68 ${ }^{13} \mathrm{C}$ NMR $\left(125 \mathrm{MHz}, \mathrm{CDCl}_{3}, 22{ }^{\circ} \mathrm{C}\right)$ spectrum of $\mathbf{1 0 p}$. 

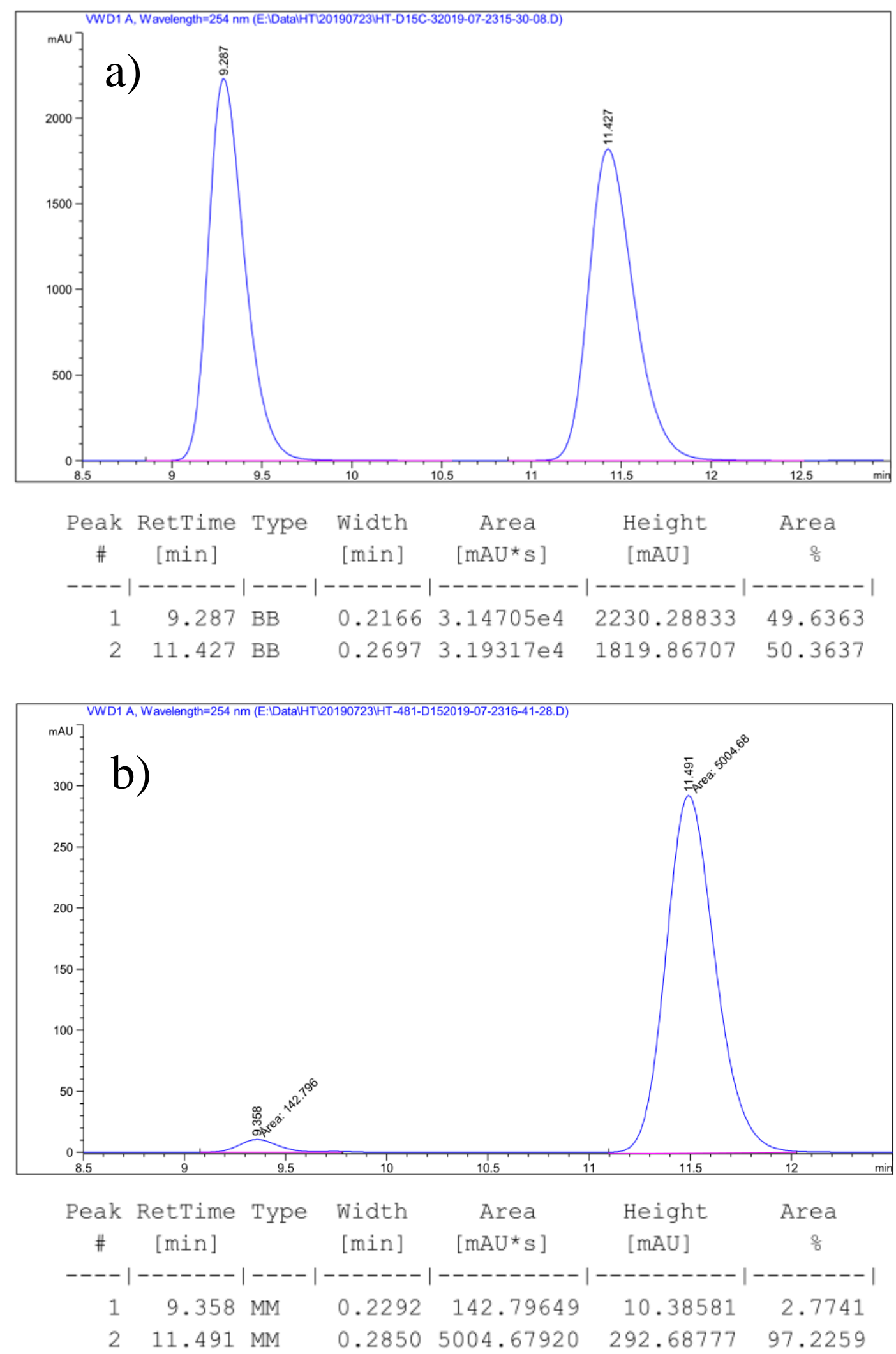

Figure S69. Chiral HPLC analysis of a) racemic 10p and b) (-)-10p (Chiralpak OD-H hexane/2-propanol 98.5/1.5, $1.0 \mathrm{~mL} / \mathrm{min}, \lambda=254 \mathrm{~nm}$ ). 

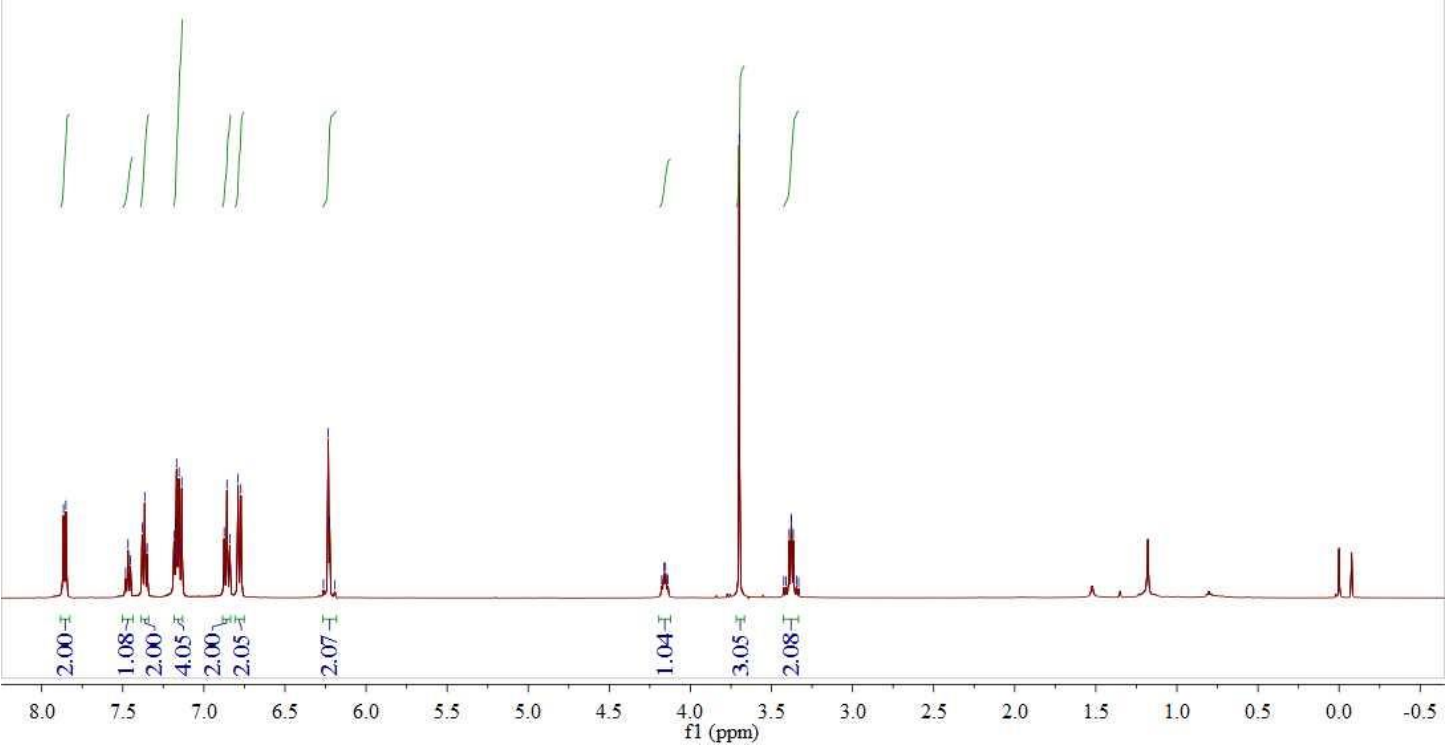

Figure S70. ${ }^{1} \mathrm{H}$ NMR $\left(500 \mathrm{MHz}, \mathrm{CDCl}_{3}, 22{ }^{\circ} \mathrm{C}\right)$ spectrum of $\mathbf{1 0 q}$.
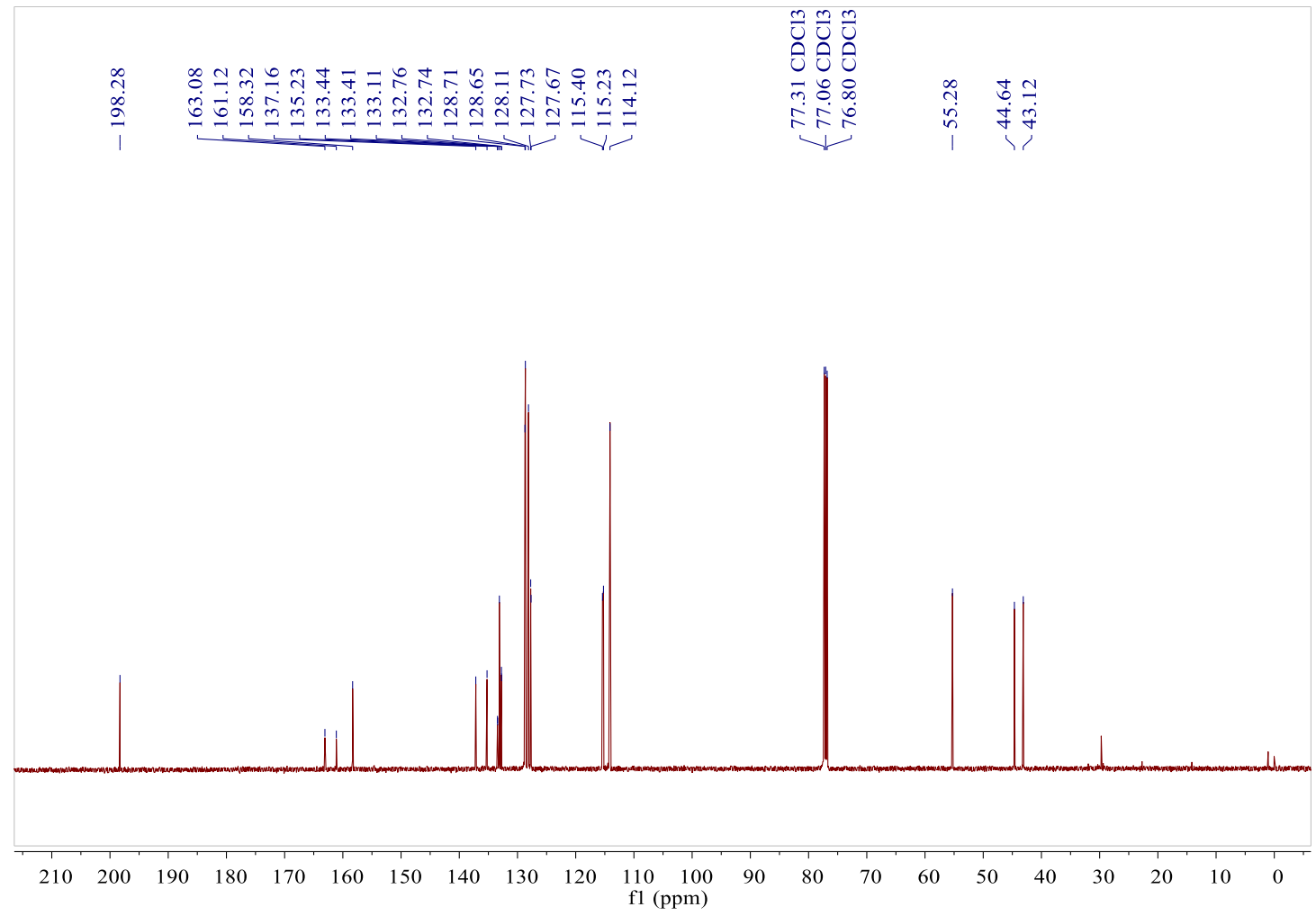

Figure S71 ${ }^{13} \mathrm{C}$ NMR $\left(125 \mathrm{MHz}, \mathrm{CDCl}_{3}, 22{ }^{\circ} \mathrm{C}\right)$ spectrum of $\mathbf{1 0 q}$. 

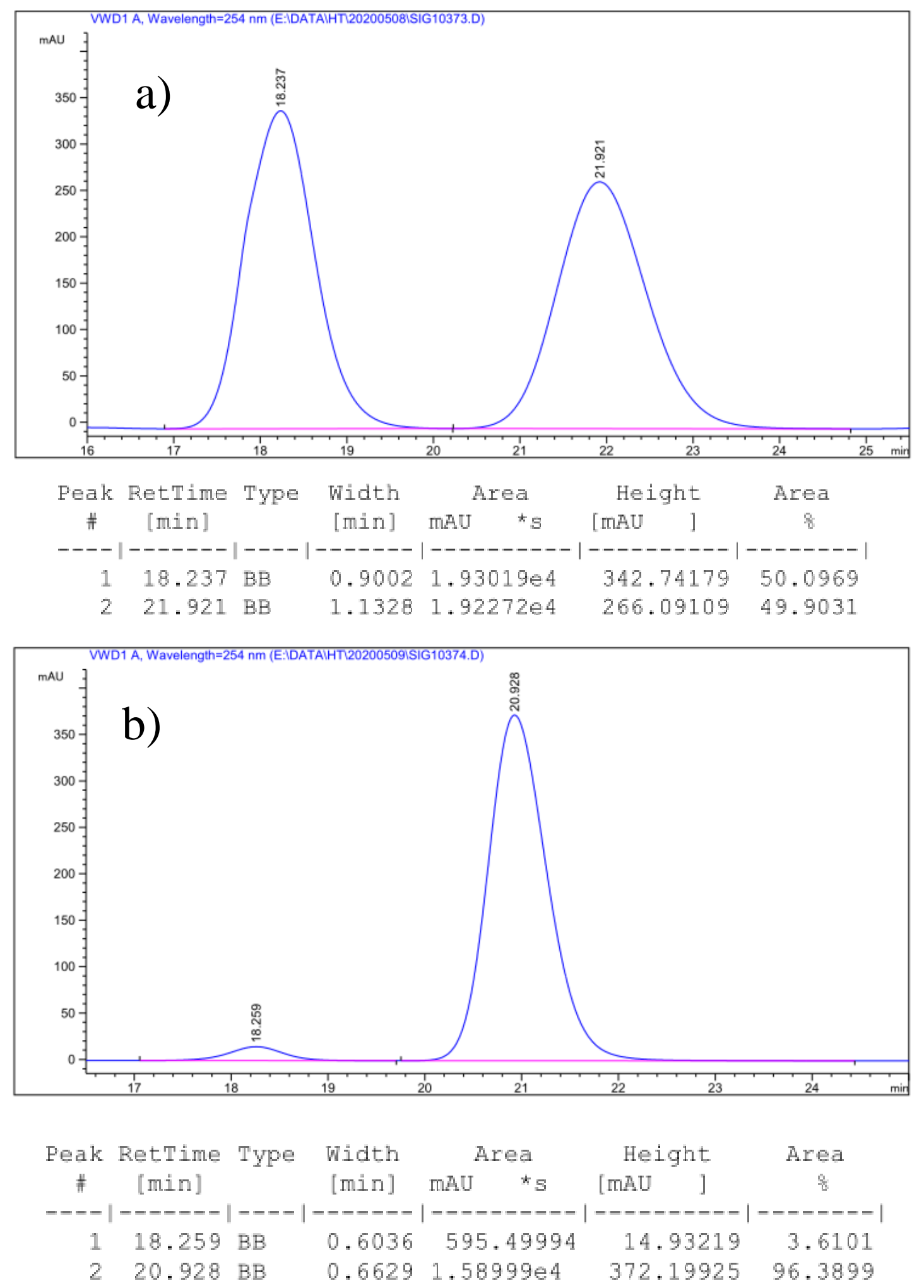

Figure S72. Chiral HPLC analysis of a) racemic 10q and b) (-)-10q (Chiralpak AS-H hexane/2-propanol 97/3, $1.0 \mathrm{~mL} / \mathrm{min}, \lambda=254 \mathrm{~nm}$ ). 


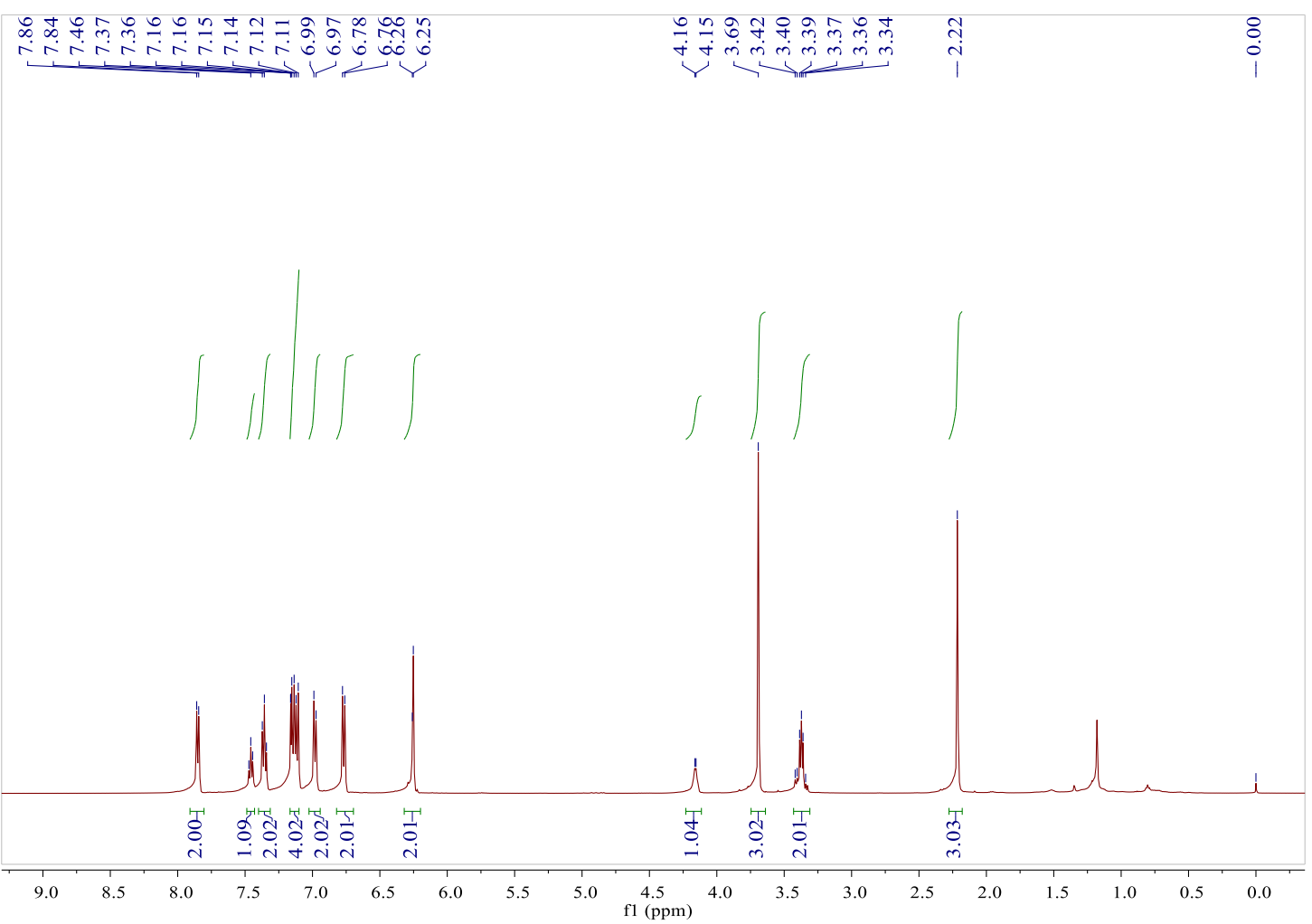

Figure S73. ${ }^{1} \mathrm{H}$ NMR $\left(500 \mathrm{MHz}, \mathrm{CDCl}_{3}, 22{ }^{\circ} \mathrm{C}\right)$ spectrum of $\mathbf{1 0 r}$

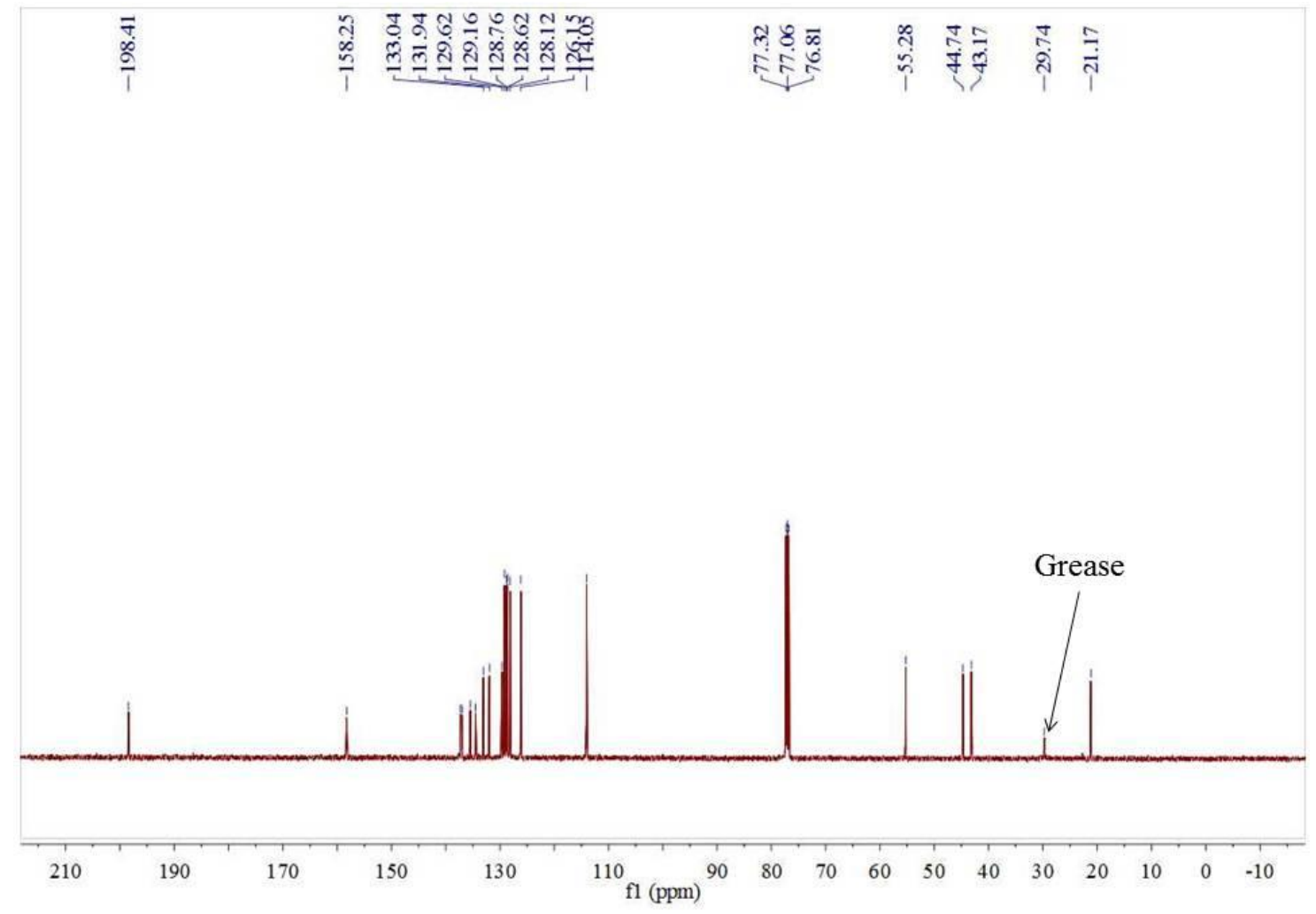

Figure $\mathbf{S 7 4}{ }^{13} \mathrm{C}$ NMR $\left(125 \mathrm{MHz}, \mathrm{CDCl}_{3}, 22{ }^{\circ} \mathrm{C}\right)$ spectrum of $\mathbf{1 0 r}$. 

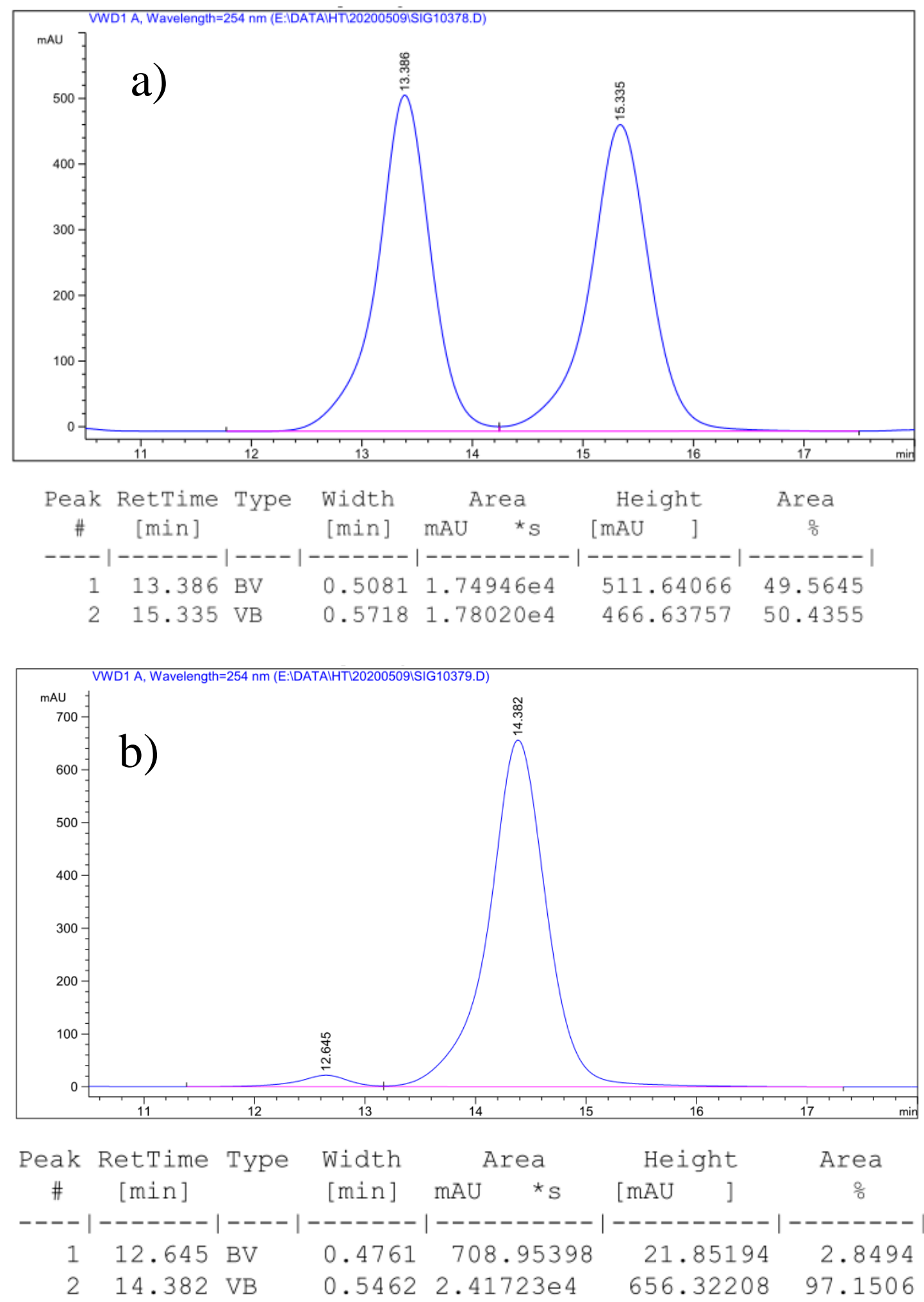

Figure S75. Chiral HPLC analysis of a) racemic 10r and b) (-)-10r (Chiralpak OD-H hexane/2-propanol 97/3, $1.0 \mathrm{~mL} / \mathrm{min}, \lambda=254 \mathrm{~nm}$ ). 


\section{References}

S1. Chi, K. W.; Addicott, C.; Arif, A. M.; Das, N.; Stang, P. J. Bidentate Ligands Capable of Variable Bond Angles in the Self-Assembly of Discrete Supramolecules. J. Org. Chem. 2003, 68, 9798-9801.

S2. Jude, H.; Sinclair, D. J.; Das, N.; Sherburn, M. S.; Stang, P. J. Self-Assembly of Supramolecular Platinum Complexes with Bis-4-pyridyl Cavitands. J. Org. Chem. 2006, $71,4155-4163$.

S3. Li, H.; Da, C.-S.; Xiao, Y.-H.; Li, X.; Su, Y.-N. Direct Asymmetric Aldol Reaction of Aryl Ketones with Aryl Aldehydes Catalyzed by Chiral BINOL-Derived Zincate Catalyst. $J$. Org. Chem. 2008, 73, 7398-7401.

S4. Gütz, C.; Hovorka, R.; Stobe, C.; Struch, N.; Topić, F.; Schnakenburg, G.; Rissanen, K.; Lützen, A. Self-Sorting Effects in the Self-Assembly of Metallosupramolecular Rhombi from Chiral BINOL-Derived Bis(pyridine) Ligands. Eur. J. Org. Chem. 2014, 206-216.

S5. Wu, T. R.; Chong, J. M. Asymmetric Conjugate Alkenylation of Enones Catalyzed by Chiral Diols. J. Am. Chem. Soc. 2007, 129, 4908-4909.

S6. Shintani, R.; Ichikawa, Y.; Takatsu, K.; Chen, F.-X.; Hayashi, T. Tuning the Chiral Environment of C2-Symmetric Diene Ligands: Development of 3,7-Disubstituted Bicyclo[3.3.1]nona-2,6-dienes. J. Org. Chem. 2009, 74, 869-873.

S7. Burger, E. C.; Tunge, J. A. Ruthenium-Catalyzed Decarboxylative Allylation of Nonstabilized Ketone Enolates. Org. Lett. 2004, 6, 2603-2605.

S8. Chai, G.-L.; Sun, A.-Q.; Zhai, D.; Wang, J.; Deng, W.-Q.; Wong, H. N. C.; Chang, J. Chiral Hydroxytetraphenylene-Catalyzed Asymmetric Conjugate Addition of Boronic Acids to Enones. Org. Lett. 2019, 21, 5040-5045.

S9. Nguyen, T. S.; Yang, M. S.; May, J. A. Experimental Mechanistic Insight into the BINOL-catalyzed Enantioselective Conjugate Addition of Boronates to Enones. Tetrahedron Lett. 2015, 56, 3337-3341.

S10. Gan K.; Ng J. S.; Sadeer A.; Pullarkat S. A. Highly Regioselective Introduction of Aryl Substituents via Asymmetric 1,4-Addition of Boronic Acids to Linear $\alpha, \beta, \gamma, \delta$-Unsaturated Ketones. Synlett 2016, 27, 254-258.

S11. Sugiura M.; Tokudomi M.; Nakajima, M. Enantioselective Conjugate Addition of Boronic Acids to Enones Catalyzed by $O$-Monoacyltartaric Acids. Chem. Commun. 2010, 46, 7799-7800.

S12. Lv J.; Zhong X.; Luo S. Taming Living Carbocations in Catalytic Direct Conjugate Addition of Simple Alkenes to $\alpha, \beta$-Enones. Chem. Eur. J. 2014, 20, 8293-8296.

S13. Le, P. Q.; Nguyen, T. S.; May, J. A. A General Method for the Enantioselective Synthesis of $\alpha$-Chiral Heterocycles. Org. Lett. 2012, 14, 6104-6107. 